\title{
A REPRESENTATION THEOREM FOR MEASURABLE RELATION ALGEBRAS
}

\author{
STEVEN GIVANT AND HAJNAL ANDRÉKA
}

\begin{abstract}
A relation algebra is called measurable when its identity is the sum of measurable atoms, where an atom is called measurable if its square is the sum of functional elements.

In this paper we show that atomic measurable relation algebras have rather strong structural properties: they are constructed from systems of groups, coordinated systems of isomorphisms between quotients of the groups, and systems of cosets that are used to "shift" the operation of relative multiplication. An atomic and complete measurable relation algebra is completely representable if and only if there is a stronger coordination between these isomorphisms induced by a scaffold (the shifting cosets are not needed in this case). We also prove that a measurable relation algebra in which the associated groups are all finite is atomic.
\end{abstract}

\section{INTRODUCTION}

The well-known pair of papers [7] and [8], by Jónsson and Tarski, were motivated by Tarski's efforts to prove that every model of his axiomatization of the calculus of relation algebras is representable, that is to say, every (abstract) relation algebra is isomorphic to a set relation algebra consisting of a universe of (binary) relations on some base set, under the standard set-theoretic operations on such relations. In the second of these papers, the authors proved several representation theorems for limited classes of relation algebras. In particular, they proved that an atomic relation algebra in which the atoms satisfy a specific "singleton inequality" is isomorphic to a set relation algebra. The singleton inequality is an inequality that is satisfied by a non-empty relation $R$ and its converse in a set relation algebra of all binary relations on a base set if and only if $R$ is a singleton relation in the sense that it has the form $R=\{(p, q)\}$ for some elements $p$ and $q$ in the base set.

Maddux [9] considerably strengthened this representation theorem. He eliminated the assumptions that the given relation algebra be atomic and that every atom satisfy the singleton inequality. Instead, he assumed only that the identity element be the sum of a set of non-zero elements satisfying the singleton inequality. Actually, he proved an even stronger version of this theorem by showing that every relation algebra in which the identity element is the sum of a set of non-zero elements satisfying the singleton inequality or a corresponding "doubleton inequality" is isomorphic to a set relation algebra. He called such relation algebras pair dense.

2010 Mathematics Subject Classification. Primary: 03G15; Secondary: 20A15.

Key words and phrases. relation algebra, group, coset, measurable atom, Boolean algebra.

This research was partially supported by Mills College and the Hungarian National Foundation for Scientific Research, Grants T30314 and T35192. 
The purpose of this paper is to prove a substantial generalization of Maddux's theorem. The task is complicated by the fact that for no natural number $n \geq 3$ is there an equation or inequality that characterizes relations consisting of at most $n$ pairs. This obstacle may be overcome by allowing oneself to use formulas from first-order logic instead of just equations and inequalities. In [2], an atom $x \leq 1$ ' is defined to be measurable if the square $x ; 1 ; x$ is the sum of a set of functions, that is to say, a set of abstract elements $f$ satisfying the functional inequality $f^{\smile} ; f \leq 1$ '. These functions turn out to be abstract versions of permutations, and the set of these permutations that are non-zero and below the square $x ; 1 ; x$ form a group. The size of the group gives a measure of the size of $x$. A relation algebra is said to be measurable if the identity element is the sum of measurable atoms, and finitely measurable if all of the atoms in this sum have finite measure.

In [2], a large class of examples of measurable set relation algebras is constructed using systems of groups and corresponding systems of isomorphisms between quotients of the groups. The resulting algebras are called (generalized) group relation algebras, and every such algebra is an example of a complete and atomic measurable relation algebra. The class of these examples, however, does not comprehend all possible examples of complete and atomic measurable relation algebras. In [1], the class of examples is expanded by using systems of cosets to "shift", or change the value, of the operation of relational composition in group relation algebras. A characterization is given in [1] of when such "shifted" group relation algebras are relation algebra, and therefore examples of complete and atomic measurable relation algebras. They are called coset relation algebras An example is given in [1] of a coset relation algebra - and therefore of an atomic, measurable relation algebrathat is not isomorphic to any set relation algebra, so not all atomic measurable relation algebras are representable in the classical sense of the word.

The purpose of the present paper is to prove that the class of coset relation algebras is adequate for the task of "representing in a wider sense" all atomic, measurable relation algebras. In the main theorem of the paper, we show that every atomic, measurable relation algebra $\mathfrak{B}$ is essentially isomorphic to a coset relation algebra $\mathfrak{C}$ in the sense that the completion (the minimal complete extension) of $\mathfrak{B}$ is isomorphic to $\mathfrak{C}$. (The passage to the completion does not change the structure of $\mathfrak{B}$, it only fills in any missing infinite sums that are needed in order to obtain isomorphism with the complete relation algebra $\mathfrak{C}$ ). In particular, every measurable relation algebra that is finite is isomorphic to a coset relation algebra. If the algebra $\mathfrak{B}$ is not finite, but is finitely measurable, then the assumption that $\mathfrak{B}$ be atomic may be dropped. We also prove that a measurable relation algebra $\mathfrak{B}$ is essentially isomorphic to a group relation algebra if and only if $\mathfrak{B}$ has a "scaffold" of atoms, and this occurs if and only if $\mathfrak{B}$ is completely representable.

Except for basic facts about groups, this article is intended to be self-contained. The definition of a relation algebra, and the relatively few basic relation algebraic laws that are needed to follow the proof in the paper are presented in Section 2. Readers who wish to learn more about the subject are recommended to look at Hirsch-Hodkinson [6], Maddux [10], or Givant [3]. 


\section{Relation Algebras}

In the next few sections, most of the calculations will involve the arithmetic of relation algebras. This section provides a review the essential results that will be needed.

A relation algebra is an algebra of the form

$$
\mathfrak{A}=\left(A,+,-, ;,^{\smile}, 1^{\prime}\right),
$$

where + and ; are binary operations called addition and relative multiplication, while - and ` are unary operations called complement and converse, and 1' is a distinguished constant called the identity element, such that the following axioms are satisfied for all elements $r, s$, and $t$ in $\mathfrak{A}$.

(R1) $r+s=s+r$.

(R2) $r+(s+t)=(r+s)+t$.

(R3) $-(-r+s)+-(-r+-s)=s$.

(R4) $r ;(s ; t)=(r ; s) ; t$.

(R5) $r ; 1^{\prime}=r$.

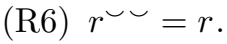

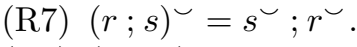

(R8) $(r+s) ; t=r ; t+s ; t$.

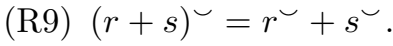

$(\mathrm{R} 10) r^{\smile} ;-(r ; s)+-s=-s$.

The axioms are commonly referred to by the following names: (R1) is the commutative law for addition, (R2) is the associative law for addition, (R3) is Huntington's law, (R4) is the associative law for relative multiplication, (R5) is the (right-hand) identity law for relative multiplication, (R6) is the first involution law, (R7) is the second involution law, (R8) is the (right-hand) distributive law for relative multiplication, (R9) is the distributive law for converse, and (R10) is Tarski's law. Under the assumption of the remaining axioms, (R10) is equivalent to the implication

$$
\text { if }(r ; s) \cdot t=0, \quad \text { then }\left(r^{\smile} ; t\right) \cdot s=0,
$$

which we shall call the cycle law. It is this form of (R10) that we shall always use. Axioms (R1)-(R3) secure that $(A,+,-)$ is a Boolean algebra. It is called the Boolean part of $\mathfrak{A}$. We shall justify a consequence of these three axioms with the phrase by Boolean algebra. The Boolean operation of multiplication · is defined in the usual way in terms of addition and complement. An element $x$ in $\mathfrak{A}$ is called a subidentity element if it is below the identity element, in symbols $x \leq 1$ '.

Whenever parentheses indicating the order of performing operations are lacking, it is understood that unary operations have priority over binary operations, and multiplications have priority over addition.

Lemma 2.1. The operation of converse is an automorphism of the Boolean part of a relation algebra. In particular, the following laws hold.

(i) $1^{\smile}=1, \quad 0^{\smile}=0, \quad 1^{\prime} \smile=1$ '.

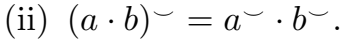

(iii) $(-a)^{-}=-\left(a^{-}\right)$.

(iv) $a \leq b$ if and only if $a^{\smile} \leq b^{\smile}$.

(v) $a=0$ if and only if $a^{\smile}=0$.

(vi) $a$ is an atom if and only if $a^{\smile}$ is an atom. 
(vii) $x^{\smile}=x$ whenever $x$ is a subidentity element.

Lemma 2.2. (i) $a ; 0=0 ; a=0$.

(ii) $1 ; 1=1$.

(iii) If $a \leq b$ and $c \leq d$, then $a ; c \leq b ; d$.

(iv) $(a ; b) \cdot c=0$ if and only if $\left(a^{\smile} ; c\right) \cdot b=0$, if and only if $\left(c ; b^{-}\right) \cdot a=0$.

(v) If $a, b$, and $c$ are atoms, then

$c \leq a ; b \quad$ if and only if $b \leq a^{\smile} ; c, \quad$ if and only if $a \leq c ; b^{\smile}$.

(vi) $a \leq a ; 1$.

(vii) $(a ; 1) \cdot(1 ; b)=a ; 1 ; b$.

(viii) If $x$ and $y$ are subidentity atoms, then

$$
1 ; x ; 1=1 ; y ; 1 \quad \text { if and only if } \quad x ; 1 ; y \neq 0 .
$$

The laws in Lemma 2.1(iv) and Lemma 2.2(iii) are known as the monotony laws for converse and relative multiplication respectively. In referring to one of these laws to justify a step in some proof, we shall usually just say by monotony. The equivalences in Lemma 2.2(iv),(v) are usually called the cycle laws and the cycle laws for atoms, respectively - as opposed to the cycle law, which is (R11) and which is just one of the implications in (iv). Again, in using these equivalences to justify some step in a proof, we shall usually just say by the cycle laws.

The operations of relative multiplication and converse are completely distributive over addition in the sense that for any two sets of elements $X$ and $Y$, if the sums $\sum X$ and $\sum Y$ exist, then the sums of the sets

$$
X ; Y=\{a ; b: a \in X \text { and } b \in Y\}, \quad Y^{\smile}=\left\{b^{\smile}: b \in Y\right\}
$$

exist, and

$$
\left(\sum X\right) ;\left(\sum Y\right)=\sum X ; Y, \quad \sum Y^{\smile}=\left(\sum Y\right)^{\smile} .
$$

In referring to one of these laws to justify a step in some proof, we shall usually just say by complete distributivity.

The domain and range of an element $a$ are defined to be the elements $(a ; 1) \cdot 1$ ' and $(1 ; a) \cdot 1$ ' respectively. Notice that they are subidentity elements. Every law about domains has a corresponding dual law about ranges. Therefore, only the law concerning domains will usually be given.

Lemma 2.3. Let $x, y$, and $z$ be subidentity atoms. Every non-zero element a $\leq$ $x ; 1 ; y$ has $x$ as its domain and $y$ as its range, and consequently the following laws hold.

(i) $x=\left(a ; a^{\smile}\right) \cdot 1^{\prime}=(a ; 1) \cdot 1^{\prime}$ and $y=\left(a^{\smile} ; a\right) \cdot 1^{\prime}=(1 ; a) \cdot 1^{\prime}$.

(ii) $x ; 1=a ; 1$ and $1 ; y=1 ; a$.

(iii) $x ; a=a$ and $a ; y=a$.

(iv) If $a \leq x ; 1 ; y$ and $b \leq y ; 1 ; z$. then $a ; b=0$ if and only if $a=0$ or $b=0$. In particular, if $a \neq 0$, then $a ; a^{\smile} \neq 0$ and $a^{\smile} ; a \neq 0$.

An element $f$ is called a function, or a functional element, if $f^{\smile} ; f \leq 1^{\prime}$. If the converse of a function $f$ is also a function, then $f$ is said to be bijective. The element $f$ is a permutation, or a permutational element, with domain $x$ if it is bijective and if its domain and range are $x$.

Lemma 2.4. Let $f, g$ be functions, and $a, b$ arbitrary elements. 
(i) $f ;(a \cdot b)=(f ; a) \cdot(f ; b)$ and $(a \cdot b) ; f^{\smile}=\left(a ; f^{\smile}\right) \cdot\left(b ; f^{\smile}\right)$.

(ii) $f ; g$ is a function.

(iii) If $a \leq f$, then $a$ is also a function.

(iv) If $f$ and $g$ are bijective, then so are $f$ and $f ; g$.

(v) If $f$ and $g$ are permutations with domain $x$, then so are $f^{\sim}$ and $f ; g$. Consequently, the permutations with domain $x$ form a group under the operations of relative multiplication and converse, with $x$ as the identity element of the group.

(vi) A function is an atom if and only if its domain is an atom.

Part (i) of the preceding lemma says that if the left-hand argument of a relative product is a function, or the right-hand argument is the converse of a function, then the operation of relative multiplication distributes over multiplication. We shall refer to this law as the distributive law for functions. It plays an extremely important role in this work.

A square is an element of the form $x ; 1 ; x$ for some subidentity element $x$, and a rectangle is an element of the form $x ; 1 ; y$ for some subidentity elements $x$ and $y$. The elements $x$ and $y$ are sometimes referred to as the sides of the rectangle

Lemma 2.5. Let $x, y, z, w$ be subidentity elements.

(i) $(x ; 1 ; y) \cdot 1^{\prime}=x \cdot y$.

(ii) $(x ; 1 ; y) \cdot(w ; 1 ; z)=(x \cdot w) ; 1 ;(y \cdot z)$.

(iii) $(x ; 1 ; y)^{\smile}=y ; 1 ; x$.

(iv) $(x ; 1 ; y) ;(y ; 1 ; z) \leq x ; 1 ; z$, and equality holds whenever $x, y$, and $z$ are atoms such that $x ; 1 ; y$ and $y ; 1 ; z$ are both non-zero.

\section{Measurable atoms}

Throughout this and the next few sections, we assume that all elements belong to an arbitrary, but fixed, relation algebra $\mathfrak{A}$ with universe $A$. In order not to have to worry about the existence of certain infinite sums, we assume that $\mathfrak{A}$ is complete. This assumption in no way restricts the applicability of the main results of the paper.

Definition 3.1. A subidentity atom $x$ is measurable if the square $x ; 1 ; x$ with side $x$ is a sum of functions. If this square is actually the sum of finitely many functions, then $x$ is said to be finitely measurable.

It turns out that the set of non-zero functions below the square $x ; 1 ; x$ of a measurable atom $x$ coincides with the set of atoms below the square, and the cardinality of this set is a measure of the "size" of $x$.

Lemma 3.2. If $x$ is a measurable atom, then an element below the square $x ; 1 ; x$ is an atom if and only if it is non-zero function.

Proof. Let $x$ be a measurable atom, and $F$ the set of functions below $x ; 1 ; x$. The definition of measurability implies that

$$
x ; 1 ; x=\sum F .
$$

If $f$ is an atom below $x ; 1 ; x$, then

$$
0<f=f \cdot(x ; 1 ; x)=f \cdot\left(\sum F\right)=\sum\{f \cdot g: g \in F\},
$$


by Boolean algebra and (1), so there must be a function $g$ in $F$ such that $f \cdot g$ is non-zero. But then $f \leq g$, because $f$ is an atom. Any element below a function is itself a function, by Lemma 2.4(iii), so $f$ is a non-zero function.

On the other hand, if $f$ is a non-zero function below $x ; 1 ; x$, then the domain of $f$ is $x$, by Lemma 2.3 , and $x$ is an atom, by assumption, so $f$ is an atom, by Lemma 2.4(vi).

The non-zero functions below the square on a measurable atom actually form a group umder the operatons of relative multiplication and converse.

Lemma 3.3. If $x$ is a measurable atom, then the set of non-zero functions below the square $x ; 1 ; x$ coincides with the set of permutations with domain $x$. This set forms a group under the operations ; and ${ }^{-}$, with identity element $x$.

Proof. If $f$ is a non-zero function below $x ; 1 ; x$, then $f$ is an atom, by Lemma 3.2. The converse of an atom is an atom, by Lemma 2.1(vi), so $f^{-}$is also an atom. Apply Lemma 3.2 again to conclude that $f^{-}$is a function, and therefore $f$ is a bijection. The element $x$ is assumed to be an atom, so every non-zero element below the square $x ; 1 ; x$ has domain and range $x$, by Lemma 2.3. In particular, the domain and range of $f$ are both $x$, so $f$ is a permutation with domain $x$.

If $g$ is an arbitrary permutation with domain $x$, then $g$ is an atom, and hence non-zero, by Lemma 2.4(vi). Furthermore,

$$
g \leq(g ; 1) \cdot(1 ; g)=(x ; 1) \cdot(1 ; x)=x ; 1 ; x,
$$

by Lemmas 2.2(vi), 2.3(ii), and 2.2(vii), so $g$ is a non-zero function below $x ; 1 ; x$. Conclusion: the set of non-zero functions below $x ; 1 ; x$ coincides with the set of permutations with domain $x$. This last set is a group under the operations of relative multiplication and converse, with $x$ as the identity element, by Lemma $2.4(\mathrm{v})$, so the same must be true of the set of non-zero functions below $x ; 1 ; x$.

The preceding lemma justifies the following definition.

Definition 3.4. The group of non-zero functions below the square on a measurable atom $x$ is denoted by $G_{x}$. The cardinality of this group is called the measure of $x$.

The following corollary is an immediate consequence of Definitions 3.1 and 3.4

Corollary 3.5. If $x$ is a measurable atom, then $x ; 1 ; x=\sum G_{x}$.

Lemma 3.6. If $x$ and $y$ are distinct measurable atoms, then the groups $G_{x}$ and $G_{y}$ are disjoint.

Proof. The squares $x ; 1 ; x$ and $y ; 1 ; y$ are disjoint, because

$$
(x ; 1 ; x) \cdot(y ; 1 ; y)=(x \cdot y) ; 1 ;(x \cdot y)=0 ; 1 ; 0=0,
$$

by Lemmas $2.5(\mathrm{ii})$ and $2.2(\mathrm{i})$. The groups $G_{x}$ and $G_{y}$ consist of non-zero elements below these respective squares, so they can have no elements in common.

Fix two measurable atoms $x$ and $y$, and let $f$ be an element in $G_{x}$, that is to say, let $f$ be a non-zero function below $x ; 1 ; x$. Define a mapping $\vartheta_{f}$ on the set

$$
A(x ; 1 ; y)=\{a \in A: a \leq x ; 1 ; y\}
$$

by stipulating that

$$
\vartheta_{f}(a)=f ; a
$$


for every $a$ in $A(x ; 1 ; y)$. The relative product $f ; a$ is called the left translation of a by $f$, so $\vartheta_{f}$ maps every element in $A(x ; 1 ; y)$ to its left translation by $f$.

Lemma 3.7. Each mapping $\vartheta_{f}$ is a permutation of the set $A(x ; 1 ; y)$, and the correspondence $(f, a) \longmapsto \vartheta_{f}(a)$ defines a left action of the group $G_{x}$ on the set $A(x ; 1 ; y)$ in the sense that

$$
\vartheta_{x}(a)=a \quad \text { and } \quad \vartheta_{g}\left(\vartheta_{f}(a)\right)=\vartheta_{g ; f}(a)
$$

for all a in $A(x ; 1 ; y)$.

Proof. Consider elements $f$ and $g$ in $G_{x}$, and $a$ in $A(x ; 1 ; y)$. We have

$$
\vartheta_{f}(a)=f ; a \leq(x ; 1 ; x) ;(x ; 1 ; y) \leq x ; 1 ; y,
$$

by the definition of $\vartheta_{f}$, the assumptions on $f$ and $a$, monotony, and Lemma 2.5(iv). Consequently, $\vartheta_{f}(a)$ belongs to the set $A(x ; 1 ; y)$. Also,

$$
\vartheta_{g}\left(\vartheta_{f}(a)\right)=g ;(f ; a)=(g ; f) ; a=\vartheta_{g ; f}(a),
$$

by the definitions of $\vartheta_{f}, \vartheta_{g}$, and $\vartheta_{g ; f}$, and the associative law; and

$$
\vartheta_{x}(a)=x ; a=a,
$$

by the definition of $\vartheta_{x}$ and Lemma 2.3(iii). Thus, the correspondence

$$
(f, a) \longmapsto \vartheta_{f}(a)
$$

does define a left action of the group $G_{x}$ on the set $A(x ; 1 ; y)$. It follows that

$$
a=\vartheta_{x}(a)=\vartheta_{f} \smile ; f(a)=\vartheta_{f} \smile\left(\vartheta_{f}(a)\right),
$$

by (2), Lemma 3.3 , and (1), and dually,

$$
a=\vartheta_{f}\left(\vartheta_{f} \smile(a)\right),
$$

so that the mappings $\vartheta_{f} \triangleleft$ and $\vartheta_{f}$ are inverses of one another. In particular, they must be one-to-one and onto, and hence permutations of the set $A(x ; 1 ; y)$.

The set $A(x ; 1 ; y)$ is closed under the binary operations of addition + and multiplication - in $\mathfrak{A}$, and also under the unary relativized complement operation $-{ }_{x ; 1 ; y}$ that is defined by

$$
-{ }_{x ; 1 ; y} a=(x ; 1 ; y) \cdot(-a)
$$

for all $a$ in $A(x ; 1 ; y)$, where $-a$ is the complement of $a$ in $\mathfrak{A}$. Under these operations, the set $A(x ; 1 ; y)$ becomes a Boolean algebra, and actually a relativization of the Boolean part of $\mathfrak{A}$. Notice that an element belonging to this relativization is an atom in $\mathfrak{A}$ just in case it is an atom in the relativization.

Lemma 3.8. The mapping $\vartheta_{f}$ is an automorphism of the relativized Boolean algebra

$$
\left(A(x ; 1 ; y),+, \cdot,-{ }_{x ; 1 ; y}\right) .
$$

In particular, an element $a \leq x ; 1 ; y$ is an atom if and only if $f ; a$ is an atom.

Proof. The mapping $\vartheta_{f}$ is a permutation of the set $A(x ; 1 ; y)$, by Lemma 3.7. The distributive law (R8) implies that $\vartheta_{f}$ preserves the operation of addition,

$$
\vartheta_{f}(a+b)=f ;(a+b)=f ; a+f ; b=\vartheta_{f}(a)+\vartheta_{f}(b) .
$$


The distributive law for functions, Lemma 2.4(i), and the assumption that $f$ is a function, imply that $\vartheta_{f}$ preserves multiplication,

$$
\vartheta_{f}(a \cdot b)=f ;(a \cdot b)=(f ; a) \cdot(f ; b)=\vartheta_{f}(a) \cdot \vartheta_{f}(b) .
$$

The element 0 is mapped to itself,

$$
\vartheta_{f}(0)=f ; 0=0,
$$

by Lemma 2.2(i). Finally, $\vartheta_{f}$ maps the unit $x ; 1 ; y$ of the relativization to itself,

$$
\vartheta_{f}(x ; 1 ; y)=f ; x ; 1 ; y=f ; 1 ; y=x ; 1 ; y,
$$

by the definition of $\vartheta_{f}$, and Lemmas 3.3 and 2.3(ii). The operation of complement in the relativization can be defined in terms of addition and multiplication, with the help of the elements 0 and $x ; 1 ; y$, so $\vartheta_{f}$ must also preserve the operation of complement in the relativization. Conclusion: $\vartheta_{f}$ is an automorphism of the relativization. Automorphisms obviously map atoms to atoms, so the second assertion of the lemma follows at once from the first one, together with the remarks preceding the lemma.

One of the main points of Lemma 3.8 is that left translation by an element $f$ in the group $G_{x}$ maps the set of atoms of $\mathfrak{A}$ that are below $x ; 1 ; y$ bijectively to itself.

Definition 3.9. For each element $a \leq x ; 1 ; y$, the left stabilizer of $a$ in $G_{x}$ under the group action of left translation is defined to be the set

$$
\left\{f \in G_{x}: f ; a=a\right\} .
$$

It will be denoted by $H_{a}$.

The next corollary is an immediate consequence of Lemma 3.7 and well-known basic facts about group actions.

Corollary 3.10. For each $a \leq x ; 1 ; y$, the left stabilizer $H_{a}$ is a subgroup of $G_{x}$. For any two elements $f$ and $g$ in $G_{x}$, we have $f ; a=g$; $a$ if and only if $f$ and $g$ are in the same left coset of $H_{a}$.

The preceding corollary implies that all elements in a coset $H_{\xi}$ of $H_{a}$ give rise to the same left translation of $a$. Write $H_{\xi} ; a$ to denote this left translation. This notation helps to avoid the cumbersome task of specifying in advance a representative $f$ of the coset $H_{\xi}$, and writing $f ; a$. In a similar vein, for any subset $X$ of $G_{x}$, write

$$
X ; a=\{f ; a: f \in X\}, \quad \text { so that } \quad \sum X ; a=\sum\{f ; a: f \in X\} .
$$

In complete analogy with the definition of $\vartheta_{f}$, for each element $g$ in $G_{y}$ one can define a mapping $\psi_{g}$ that sends every element $a$ below $x ; 1 ; y$ to its right translation by $g$,

$$
\psi_{g}(a)=a ; g .
$$

The mapping $\psi_{g}$ is a permutation of the set $A(x ; 1 ; y)$, and the correspondence $(g, a) \longmapsto \psi_{g}(a)$ defines a right action of the group $G_{y}$ on the set $A(x ; 1 ; y)$. The mapping $\psi_{g}$ is an automorphism of the relativized Boolean algebra corresponding to $A(x ; 1 ; y)$. In particular, an element $a \leq x ; 1 ; y$ is an atom if and only if $a ; g$ is an atom. The right stabilizer of an element $a \leq x ; 1 ; y$ under the group action of right translation is defined to be the set

$$
\left\{g \in G_{y}: a ; g=a\right\},
$$


and is denoted by $K_{a}$. The right stabilizer proves to be a subgroup of $G_{y}$, and for any two elements $f$ and $g$ in $G_{y}$, the right translations $a ; f$ and $a ; g$ are equal just in case $f$ and $g$ are in the same right coset of $K_{a}$. Consequently, if $K_{\eta}$ is a right coset of $K_{a}$, then it makes sense to write $a ; K_{\eta}$ to denote the uniquely determined element that is the right translation of $a$ by elements in $K_{\eta}$.

Every result about left translations and left stabilizers has a corresponding dual result about right translations and right stabilizers. In general, we will usually formulate only the left-hand version, while allowing ourselves to refer to the righthand versions in later proofs that require it. The following easy lemma gives an example.

Lemma 3.11. Let $x, y$, and $z$ be measurable atoms. If $a \leq x ; 1 ; y$ and $b \leq y ; 1 ; z$, then $H_{a} \subseteq H_{a ; b}$.

Proof. If $f$ is in $H_{a}$, then $f ; a=a$, by the definition of $H_{a}$, and therefore

$$
f ; a ; b=a ; b .
$$

It follows that $f$ is in $H_{a ; b}$.

\section{LEFT-REGULAR AND RIGHT-REGULAR ELEMENTS}

A special class of elements called regular elements plays an important role in the subsequent discussion. As will be seen, the prototypical regular element is an atom. More generally, if $a$ is an atom below $x ; 1 ; y$, and if $M$ is a subgroup of $G_{x}$ that extends the left stabilizer $H_{a}$ of $a$, then the element

$$
b=\sum M ; a=\sum\{f ; a: f \in M\}
$$

is a regular element.

Many of the properties of regular elements hold for broader classes of elements called left-regular elements and right-regular elements respectively. We begin with a study of these elements.

Definition 4.1. Let $x$ and $y$ be measurable atoms. An element $a \leq x ; 1 ; y$ is called left-regular or right-regular respectively, according to whether

$$
a ; a^{\smile}=\sum H_{a} \quad \text { or } \quad a^{\smile} ; a=\sum K_{a},
$$

and $a$ is called regular if it is both left and right-regular.

The next lemma and the remarks following it are intended to clarify this definition.

Lemma 4.2. Let $x$ and $y$ be measurable atoms. For any elements $a$ and $b$ below $x ; 1 ; y$, there are uniquely determined sets $E \subseteq G_{x}$ and $F \subseteq G_{y}$ such that

$$
a ; b^{\smile}=\sum E \quad \text { and } \quad a^{\smile} ; b=\sum F .
$$

If $a=b \neq 0$, then $E$ and $F$ contain $x$ and $y$ respectively and are closed under the operation of converse.

Proof. Assume

$$
0 \leq a, b \leq x ; 1 ; y .
$$

Use (1), Lemma 2.1(i), monotony, and Lemma 2.5(iii) to obtain

$$
0 \leq b^{-} \leq(x ; 1 ; y)^{\smile}=y ; 1 ; x .
$$


Use (1), (2), monotony, and Lemma 2.5(iv) to arrive at

$$
0 \leq a ; b^{-} \leq(x ; 1 ; y) ;(y ; 1 ; x) \leq x ; 1 ; x .
$$

The set $A(x ; 1 ; x)$ of elements below $x ; 1 ; x$ is a Boolean algebra with unit $x ; 1 ; x$, under the operations of addition and complement relativized to $x ; 1 ; x$, by the remarks preceding Lemma 3.8. The unit $x ; 1 ; x$ is the sum of the set $G_{x}$, by Corollary 3.5 and the assumption that $x$ is measurable. Moreover, $G_{x}$ coincides with the set of atoms that are below $x ; 1 ; x$, by Lemma 3.2. It follows that the relativized Boolean algebra $A(x ; 1 ; x)$ is atomic, and its set of atoms is $G_{x}$. In an atomic Boolean algebra, each element is the sum of a uniquely determined set of atoms. Combine these remarks to conclude that each element below $x ; 1 ; x$ is the sum of a uniquely determined subset of $G_{x}$. This applies in particular to the element $a ; b^{\smile}$, by (3), so there must be a unique subset $E$ of $G_{x}$ such that

$$
a ; b^{\smile}=\sum E \text {. }
$$

Assume next that $a=b \neq 0$, and observe that $a ; a^{\smile} \neq 0$, by (1), (2) (with $a$ in place of $b$ ), and Lemma 2.3(iv). The element $a ; a^{\smile}$ is left fixed by converse, because

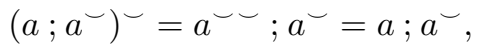

by the involution laws (R7) and (R6). Consequently,

$$
\sum E=a ; a^{\smile}=\left(a ; a^{\smile}\right)^{\smile}=\left(\sum E\right)^{\smile}=\sum\left\{f^{\smile}: f \in E\right\},
$$

by (4) (with $a$ in place of $b$ ), (5), (4), and complete distributivity. Two sums of sets of atoms are equal just in case the sets themselves are equal, so (6) implies that

$$
E=\left\{f^{\smile}: f \in E\right\} .
$$

Thus, the set $E$ is closed under converse. The element $a$ is non-zero, by assumption, and below $x ; 1 ; y$, by (1), so it has as its domain the atom $x$, by Lemma 2.3 . It follows that $x \leq a ; a^{\smile}$, by the assumption on $x$ and Lemma 2.3(i), and therefore $x$ is in $E$, by the definition of $E$.

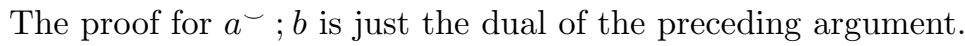

Fix an element $a$ below $x ; 1 ; y$. Because of the preceding lemma there is always a unique set of atoms $E \subseteq G_{x}$ such that $a ; a^{\smile}=\sum E$. In what follows, this set will be denoted by $X_{a}$. Similarly, there is a unique set of atoms $F \subseteq G_{y}$ such that $a^{\smile} ; a=\sum F$, and this set will be denoted by $Y_{a}$. This notation and the definitions of left- and right-regular elements immediately imply the following corollary.

Corollary 4.3. An element $a \leq x ; 1 ; y$ is left-regular or right-regular if and only if $X_{a}=H_{a}$ or $Y_{a}=K_{a}$ respectively.

Lemma 4.4. A left-regular or right-regular element is always non-zero.

Proof. The stabilizer $H_{0}$ of the zero element 0 is $G_{x}$, because $f ; 0=0$ for all $f \in G_{x}$, by Lemma 2.2(i). Notice that this stabilizer is not empty since it contains, for example, the element $x$. On the other hand,

$$
0 ; 0^{-}=0=\sum \varnothing,
$$

by Lemma $2.2(\mathrm{i})$, so the set $X_{0}$ of atoms below $0 ; 0^{-}$is empty. It follows that the sets $H_{0}$ and $X_{0}$ cannot be equal, and therefore 0 cannot be left-regular, by Corollary 4.3. A dual argument proves the corresponding result for right-regular elements. 
In the remainder of this section, we shall usually only formulate lemmas and theorems for left-regular element, leaving the formulations and proofs of the dual results for right-regular elements to the reader. When there is a need to refer to such a result, we shall simply refer to "the right-regular version of ...".

Lemma 4.5. Suppose $x$ and $y$ are measurable atoms, and $0<a \leq x ; 1 ; y$.

(i) An element $f$ in $G_{x}$ belongs to $X_{a}$ if and only if $(f ; a) \cdot a \neq 0$.

(ii) $H_{a} \subseteq H_{a ; a} \subseteq X_{a} \subseteq G_{x}$.

Proof. For any element $f$ in $G_{x}$,

$$
\begin{aligned}
f \in X_{a} \quad \text { if and only if } & f \leq a ; a^{-}, \\
\text {if and only if } & f \cdot\left(a ; a^{-}\right) \neq 0, \\
\text { if and only if } & \left(f ;\left(a^{-}\right)^{-}\right) \cdot a \neq 0, \\
\text { if and only if } & (f ; a) \cdot a \neq 0,
\end{aligned}
$$

by the definition of $X_{a}$, the fact that $f$ is an atom (by Lemmas 3.2, 3.3, and the definition of $G_{x}$ ), the cycle laws, and the first involution law. This proves (i).

To establish the first inclusion in (ii), recall that

$$
a^{\smile} \leq(x ; 1 ; y)^{-}=y ; 1 ; x,
$$

by the assumption on $a$, monotony, and Lemma 2.5(iii). Apply Lemma 3.11 (with $a^{\smile}$ and $x$ in place of $b$ and $z$ respectively) to arrive at the desired inclusion. To establish the second inclusion, assume that $f$ is in $H_{a ; a} \smile$. The second assertion of Lemma 4.2 implies that $x$ is below $a ; a^{\smile}$. Consequently,

$$
f=f ; x \leq f ; a ; a^{\smile}=a ; a^{\smile}=\sum X_{a},
$$

by Lemma 3.3, monotony, the assumption that $f$ is in the stabilizer of $a ; a^{\smile}$, and the definition of $X_{a}$. Since $f$ is an atom, and $X_{a}$ a set of atoms, it follows from (1) that $f$ must belong to $X_{a}$. The final inclusion in (ii) is a consequence of the definition of $X_{a}$.

It is of some interest to conclude from Lemma 4.5 that the set $X_{a}$ is a union of left cosets of $H_{a}$ in $G_{x}$. Indeed, if $f$ and $g$ are in the same left coset of $H_{a}$, then $f ; a=g ; a$ by Corollary 3.10, and therefore

$$
(f ; a) \cdot a \neq 0 \text { if and only if }(g ; a) \cdot a \neq 0 .
$$

It follows from this equivalence and part (i) of the preceding lemma that $f$ is in $X_{a}$ if and only if $g$ is in $X_{a}$. In other words, if one element of a left coset of $H_{a}$ belongs to $X_{a}$, then the entire coset is included in $X_{a}$.

Lemma 4.6. Let $x$ and $y$ be measurable atoms. For each non-zero $a \leq x ; 1 ; y$, the following are equivalent.

(i) a is left-regular.

(ii) For any $f$ in $G_{x}$, either $f ; a=a$ or $(f ; a) \cdot a=0$.

(iii) For any $f$ and $g$ in $G_{x}$, either $f ; a=g ; a$ or $(f ; a) \cdot(g ; a)=0$.

Proof. Let $f$ and $g$ be elements of $G_{x}$, and $a$ a non-zero element below $x ; 1 ; y$. To establish the implication from (i) to (ii), assume that $a$ is left-regular, and observe that

$$
H_{a}=X_{a},
$$


by Corollary 4.3. If $f$ is in $H_{a}$, then $f ; a=a$, by definition of the left stabilizer, and if $f$ is not in $H_{a}$, then $f$ is not in $X_{a}$, by (1), and consequently $(f ; a) \cdot a=0$, by Lemma 4.5(i). Thus, (ii) holds.

To derive (iii) from (ii), observe first that

$$
f ; a=g ; a \quad \text { if and only if } \quad g^{\smile} ; f ; a=a .
$$

Indeed, if $f ; a=g ; a$, then

$$
g^{\smile} ; f ; a=g^{\smile} ; g ; a=x ; a=a,
$$

by Lemmas 3.3 and 2.3(iii). On the other hand, if $g^{\smile} ; f ; a=a$, then $g^{\smile} ; f$ is in the left stabilizer $H_{a}$ of $a$, so that $f$ and $g$ must belong to the same left coset of $H_{a}$. Use Corollary 3.10 to conclude that $f ; a=g ; a$. From (2), (ii) applied to the element $g^{-} ; f$, and the cycle laws, it follows that

$$
\begin{array}{rll}
f ; a \neq g ; a \quad \text { if and only if } & g^{\smile} ; f ; a \neq a, \\
& \text { if and only if } & \left(g^{\smile} ; f ; a\right) \cdot a=0, \\
& \text { if and only if } & (g ; a) \cdot(f ; a)=0 .
\end{array}
$$

The implication from (iii) to (ii) is trivial: just take $g$ to be the element $x$, and use Lemma 2.3(iii).

Finally, to derive (i) from (ii), assume that (ii) holds. Certainly, $H_{a}$ is included in the set $X_{a}$, by Lemma 4.5(ii). For the reverse inclusion, consider an element $f$ in $X_{a}$. Use Lemma 4.5(i) to see that $(f ; a) \cdot a \neq 0$, and then invoke (ii) to obtain $f ; a=a$. This implies that $f$ is in the left stabilizer $H_{a}$, so $X_{a}$ is included in $H_{a}$. Thus, (1) holds, so $a$ is left-regular, by Corollary 4.3.

The next corollary implies that in a measurable relation algebra, atoms are always regular elements. This will play a very important role in the proof of the representation theorem for measurable relation algebras.

Corollary 4.7. Let $x$ and $y$ be measurable atoms. Every atom below $x ; 1 ; y$ is regular.

Proof. Let $a$ be an atom below $x ; 1 ; y$. For each $f$ in $G_{x}$ the left translation $f ; a$ is also an atom by Lemma 3.8, so $f ; a=a$ or $(f ; a) \cdot a=0$. Apply Lemma 4.6 to conclude that $a$ is left-regular. A dual argument, involving the version of Lemma 4.6 that applies to right-regular elements, shows that $a$ is right-regular. Consequently, $a$ is regular.

Lemma 4.8. Let $x$ and $y$ be measurable atoms, and $a$ and $b$ left-regular elements below $x ; 1 ; y$. If $a \leq b$, then $H_{a} \subseteq H_{b}$.

Proof. If $a \leq b$, then

$$
\sum H_{a}=\sum X_{a}=a ; a^{\smile} \leq b ; b^{\smile}=\sum X_{b}=\sum H_{b},
$$

by Corollary 4.3 and the assumption that $a$ is left-regular, the definition of $X_{a}$, the assumption that $a \leq b$ and monotony, the definition of $X_{b}$, and Corollary 4.3 and the assumption that $b$ is left-regular. The desired inclusion follows from (1) and the fact that $H_{a}$ and $H_{b}$ are sets of atoms.

Suppose $x$ and $y$ are measurable atoms and $0<a \leq x ; 1 ; y$. Fix a left coset system in $G_{x}$ of the left stabilizer $H_{a}$, and denote it by $\left\langle H_{a, \xi}: \xi<\kappa_{a}\right\rangle$ (where $\kappa_{a}$ is, say, an ordinal number that is defined to coincide with the set of its predecessors). 
When no confusion will arise, we shall drop the reference to $a$ and write simply $\left\langle H_{\xi}: \xi<\kappa\right\rangle$. Similarly, fix a right coset system in $G_{y}$ of the right stabilizer $K_{a}$, and denote it by $\left\langle K_{a, \eta}: \eta<\lambda_{a}\right\rangle$ or simply by $\left\langle K_{\eta}: \eta<\lambda\right\rangle$. The next lemma uses the notation $H_{\xi} ; a$ that was introduced after Corollary 3.10 to denote the element $f ; a$ for $f$ in $H_{\xi}$.

Lemma 4.9 (First Partition Lemma). Let $x$ and $y$ be measurable atoms. If $a \leq$ $x ; 1 ; y$ is left-regular, then $\left\langle H_{\xi} ; a: \xi<\kappa\right\rangle$ forms a partition of $x ; 1 ; y$.

Proof. It must be shown that the elements $H_{\xi} ; a$ are non-zero, pairwise disjoint, and sum to $x ; 1 ; y$. They are pairwise distinct by Corollary 3.10 , and therefore pairwise disjoint by Lemma 4.6(iii). The left-regular element $a$ is non-zero, by Lemma 4.4, so its translation $H_{\xi} ; a$ is non-zero by Lemma 3.8. Finally,

$$
\begin{aligned}
x ; 1 ; y=x ; 1 ; a=x ; 1 ; x ; a=( & \left.\sum G_{x}\right) ; a \\
& =\sum\left\{h ; a: h \in G_{x}\right\}=\sum\left\{H_{\xi} ; a: \xi<\kappa\right\},
\end{aligned}
$$

by Lemmas 2.3(ii),(iii), Corollary 3.5, and complete distributivity.

Corollary 4.10. Let $x$ and $y$ be measurable atoms, and $a \leq x ; 1 ; y$ a left-regular element. If $X$ and $Y$ are unions of left cosets of $H_{a}$, then

$$
\sum X ; a \leq \sum Y ; a \quad \text { if and only if } \quad X \subseteq Y
$$

and consequently,

$$
\sum X ; a=\sum Y ; a \quad \text { if and only if } \quad X=Y .
$$

Proof. The proof of the implication from right to left in the first assertion is trivial. To prove the reverse implication, assume that

$$
\sum X ; a \leq \sum Y ; a,
$$

and consider a left coset $H_{\xi}$ that is included in $X$. Obviously,

$$
H_{\xi} ; a \leq \sum X ; a \leq \sum Y ; a,
$$

by (1) and the fact that the element $H_{\xi} ; a$ belongs to the set $X ; a$. Two left translations of $a$ by cosets of $H_{a}$ are either equal or disjoint, by Lemma 4.9, so there must be a left coset $H_{\eta}$ included in $Y$ such that $H_{\xi} ; a=H_{\eta} ; a$. Distinct left cosets of $H_{a}$ give rise to disjoint left translations of $a$, again by Lemma 4.9, so $\xi=\eta$. Thus, every left coset of $H_{a}$ that is included in $X$ is also included in $Y$, and therefore $X$ must be included in $Y$. This proves the first assertion of the corollary. The second is an immediate consequence of the first.

A sense of the importance of Partition Lemma 4.9 can be gained from the following consequence.

Lemma 4.11 (Atomic Partition Lemma). Let $x$ and $y$ be measurable atoms. If $a \leq x ; 1 ; y$ is an atom, then $\left\langle H_{\xi} ; a: \xi<\kappa\right\rangle$ is a listing of the distinct atoms below $x ; 1 ; y$ and these atoms sum to $x ; 1 ; y$. Consequently, every element below $x ; 1 ; y$ is the sum of a unique subset of these atoms.

Proof. An atom $a$ below $x ; 1 ; y$ is a regular element, by Corollary 4.7, so the elements in the system

$$
\left\langle H_{\xi} ; a: \xi \leq \kappa\right\rangle
$$


form a partition of $x ; 1 ; y$, by Partition Lemma 4.9. In particular, they are mutually disjoint and sum to the unit $x ; 1 ; y$ of the relativized Boolean algebra $A(x ; 1 ; y)$. Moreover, they are all atoms, by Lemma 3.8. It follows by Boolean algebra that the relativized Boolean algebra $A(x ; 1 ; y)$ is atomic, and therefore each of its elements is the sum of a unique set of atoms from (1).

The preceding lemma says that if there is an atom $a$ below $x ; 1 ; y$, then every left translation of $a$ is again an atom and these atoms partition $x ; 1 ; y$. The same is of course true for the right translations of $a$.

Suppose $x$ and $y$ are measurable atoms, and $a$ and $b$ left-regular elements below $x ; 1 ; y$ with $a \leq b$. By Lemma 4.8, the left stabilizer $H_{a}$ is a subgroup of the left stabilizer $H_{b}$. Let

$$
\left\langle H_{a, \xi}: \xi<\kappa\right\rangle \quad \text { and } \quad\left\langle H_{b, \eta}: \eta<\lambda\right\rangle
$$

be left coset systems for $H_{a}$ and $H_{b}$ respectively in $G_{x}$. As is well known from group theory, there must be a partition $\left\langle\Gamma_{\eta}: \eta<\lambda\right\rangle$ of the index set $\{\xi: \xi<\kappa\}$ such that

$$
H_{b, \eta}=\bigcup\left\{H_{a, \xi}: \xi \in \Gamma_{\eta}\right\}
$$

for each $\eta<\lambda$. The next lemma, a generalization of the First Partition Lemma, refers to these assumptions.

Lemma 4.12 (Second Partition Lemma). Let $x$ and $y$ be measurable atoms, and $a$ and $b$ left-regular elements below $x ; 1 ; y$. If $a \leq b$, then $\left\langle H_{a, \xi} ; a: \xi \in \Gamma_{\eta}\right\rangle$ is a partition of $H_{b, \eta} ; b$, and in particular,

$$
H_{b, \eta} ; b=\sum H_{b, \eta} ; a=\sum\left\{H_{a, \xi} ; a: \xi \in \Gamma_{\eta}\right\}
$$

for each $\eta<\lambda$.

Proof. By assumption, $a \leq b$. Also, for each $\xi$ in $\Gamma_{\eta}$, the left coset $H_{a, \xi}$ is a subset of the left coset $H_{b, \eta}$, by the remarks preceding the lemma. Use monotony and Corollary 3.10 to obtain

$$
H_{a, \xi} ; a \leq H_{a, \xi} ; b=H_{b, \eta} ; b .
$$

The system $\left\langle H_{b, \eta} ; b: \eta<\lambda\right\rangle$ is a partition of $x ; 1 ; y$, by Partition Lemma 4.9, so the elements $H_{b, \zeta} ; b$ and $H_{b, \eta} ; b$ are disjoint for indices $\zeta, \eta<\lambda$ with $\zeta \neq \eta$. Consequently, for $\xi$ in $\Gamma_{\zeta}$,

$$
\left(H_{a, \xi} ; a\right) \cdot\left(H_{b, \eta} ; b\right) \leq\left(H_{a, \xi} ; b\right) \cdot\left(H_{b, \eta} ; b\right)=\left(H_{b, \zeta} ; b\right) \cdot\left(H_{b, \eta} ; b\right)=0,
$$

by (1) (with $\zeta$ in place of $\eta$ ) and Boolean algebra. The system

$$
\left\langle H_{a, \xi} ; a: \xi<\kappa\right\rangle
$$

is also a partition of $x ; 1 ; y$, by Lemma 4.9 , so the elements of this system are non-zero, mutually disjoint, and

$$
\sum\left\{H_{a, \xi} ; a: \xi<\kappa\right\}=x ; 1 ; y .
$$

Multiply both sides of this last equation by $H_{b, \eta} ; b$, and use (1), (2), and Boolean algebra to obtain

$$
\sum\left\{H_{a, \xi} ; a: \xi \in \Gamma_{\eta}\right\}=H_{b, \eta} ; b .
$$

Conclusion: $\left\langle H_{a, \xi}: \xi \in \Gamma_{\eta}\right\rangle$ is a partition of $H_{b, \eta} ; b$. 
The second assertion of the lemma is an almost immediate consequence of the first:

$$
H_{b, \eta} ; b=\sum\left\{H_{a, \xi} ; a: \xi \in \Gamma_{\eta}\right\}=\sum\left(\bigcup\left\{H_{a, \xi}: \xi \in \Gamma_{\eta}\right\}\right) ; a=\sum H_{b, \eta} ; a,
$$

by (4), complete distributivity, and the remarks preceding the lemma.

Non-zero products of regular elements play an important role in the analysis of the behavior of regular elements.

Lemma 4.13 (First Product Lemma). Let $x$ and $y$ be measurable atoms, and $a$ and $b$ left-regular elements below $x ; 1 ; y$. If $a \cdot b \neq 0$, then $a \cdot b$ is left-regular and $H_{a \cdot b}=H_{a} \cap H_{b}$.

Proof. Observe that

$$
(a \cdot b) ;(a \cdot b)^{\smile}=(a \cdot b) ;\left(a^{\smile} \cdot b^{\smile}\right) \leq\left(a ; a^{\smile}\right) \cdot\left(b ; b^{\smile}\right),
$$

by Lemma 2.1(ii) and monotony, so

$$
\sum X_{a \cdot b} \subseteq\left(\sum X_{a}\right) \cdot\left(\sum X_{b}\right)
$$

by the definitions of the sets $X_{a \cdot b}, X_{a}$, and $X_{b}$. Since these are all sets of atoms, it follows from (1) by Boolean algebra that

$$
X_{a \cdot b} \subseteq X_{a} \cap X_{b}
$$

Use Lemma 4.5(ii) (with $a \cdot b$ in place of $a$ ), the assumption that $a \cdot b \neq 0$, (2), the assumed left-regularity of $a$ and $b$, and Corollary 4.3 to obtain

$$
H_{a \cdot b} \subseteq X_{a \cdot b} \subseteq X_{a} \cap X_{b}=H_{a} \cap H_{b} .
$$

On the other hand, if $f$ is in $H_{a} \cap H_{b}$, then $f$ is also in $H_{a \cdot b}$, because

$$
f ;(a \cdot b)=(f ; a) \cdot(f ; b)=a \cdot b,
$$

by the distributive law for functions. Consequently,

$$
H_{a} \cap H_{b} \subseteq H_{a \cdot b} .
$$

Combine (3) with (4) to arrive at

$$
H_{a \cdot b}=X_{a \cdot b}=H_{a} \cap H_{b} .
$$

The left-regularity of the product $a \cdot b$ is an immediate consequence of the first of these equalities and Corollary 4.3 (with $a \cdot b$ in place of $a$ ).

Lemma 4.14. Let $x$ and $y$ be measurable atoms, and $a$ and $b$ left-regular elements below $x ; 1 ; y$. If $a \cdot b \neq 0$, then

$$
H_{a} \subseteq H_{b} \quad \text { if and only if } \quad a \leq b,
$$

and consequently

$$
H_{a}=H_{b} \quad \text { if and only if } \quad a=b .
$$

Proof. Assume that $a \cdot b \neq 0$. If $a \leq b$, then $H_{a}$ is included in $H_{b}$, by Lemma 4.8. To establish the reverse implication, suppose that $H_{a} \subseteq H_{b}$. Use this assumption, the assumption that $a \cdot b \neq 0$, and Product Lemma 4.13 to see that $a \cdot b$ is a left-regular element, and that

$$
H_{a \cdot b}=H_{a} \cap H_{b}=H_{a} .
$$


Let $\left\langle f_{\xi}: \xi<\kappa\right\rangle$ be a system of representatives for the left cosets of $H_{a}$ in $G_{x}$. Partition Lemma 4.9 (with $f_{\xi}$ in placed of $H_{\xi}$ ) says that

$$
\left\langle f_{\xi} ; a: \xi<\kappa\right\rangle
$$

is a partition of $x ; 1 ; y$. Now $\left\langle f_{\xi}: \xi<\kappa\right\rangle$ is also a system of representatives for the left cosets of $H_{a \cdot b}$, by (1), so

$$
\left\langle f_{\xi} ;(a \cdot b): \xi<\kappa\right\rangle
$$

is a partition of $x ; 1 ; y$, by Lemma 4.9 and the left-regularity of $a \cdot b$. Since

$$
f_{\xi} ;(a \cdot b) \leq f_{\xi} ; a
$$

for each $\xi$, by monotony, the partitions in (2) and (3) must be equal. Therefore, equality must actually hold in (4). Use this observation and the distributive law for functions to obtain

$$
f_{\xi} ; a=f_{\xi} ;(a \cdot b)=\left(f_{\xi} ; a\right) \cdot\left(f_{\xi} ; b\right) .
$$

It follows that

$$
f_{\xi} ; a \leq f_{\xi} ; b .
$$

Take $\xi=0$ in this inequality, and use Lemma 2.3(iii), together with the convention that $f_{0}=x$, to arrive at $a \leq b$. This completes the proof of the first equivalence in the lemma.

The second equivalence is an immediate consequence of the first.

The preceding lemma leads naturally to the question, for two left-regular elements $a$ and $b$ below $x ; 1 ; y$, when is the product $a \cdot b$ non-zero? A necessary and sufficient condition for this to happen is given below in the Second Product Lemma.

The next lemma says that any translation, left or right, of a left-regular element $a$ is again left-regular, and the left stabilizer of such a translation can be computed from the left stabilizer of $a$.

Lemma 4.15 (First Translation Lemma). Let $x$ and $y$ be measurable atoms, and $a \leq x ; 1 ; y$ a left-regular element.

(i) For every $f$ in $G_{x}$, the left translation $f$; $a$ is left-regular, and its left stabilizer is

$$
H_{f ; a}=f ; H_{a} ; f^{\smile} .
$$

If $H_{a}$ is a normal subgroup of $G_{x}$, then $H_{f ; a}=H_{a}$.

(ii) For every element $g$ in $G_{y}$, the right translation $a ; g$ is left-regular and its left stabilizer is $H_{a ; g}=H_{a}$.

Proof. Consider an element $f$ in $G_{x}$. Use the definition of the set $X_{f ; a}$, the second involution law and the associative law for relative multiplication, the definition of the set $X_{a}$, the assumed left-regularity of the element $a$, together with Corollary 4.3, and complete distributivity to obtain

$$
\begin{aligned}
\sum X_{f ; a}=(f ; a) ;(f ; a)^{\smile}=f ; a ; a^{\smile} ; f^{\smile}=f ;\left(\sum X_{a}\right) ; f^{\smile} & \\
& =f ;\left(\sum H_{a}\right) ; f=\sum\left(f ; H_{a} ; f^{\smile}\right) .
\end{aligned}
$$

It is easy to check, and it follows from Lemma 3.7 and group theory, that the left stabilizer of the left translation $f ; a$ is the subgroup

$$
H_{f ; a}=f ; H_{a} ; f^{\smile} \text {. }
$$


Combine (1) with (2) to arrive at

$$
\sum X_{f ; a}=\sum H_{f ; a} .
$$

Since $X_{f ; a}$ and $H_{f ; a}$ are both sets of atoms, the preceding equation implies that the two sets must be equal. Use Corollary 4.3 (with $f ; a$ in place of $a$ ) to conclude that $f ; a$ is left-regular. If, in addition, $H_{a}$ is a normal subgroup of $G_{x}$, then this subgroup must coincides with $f ; H_{a} ; f^{\sim}$, and therefore also with $H_{f ; a}$, by (2). This proves (i).

To prove (ii), assume that $g$ is in $G_{y}$. Use the definition of the set $X_{a ; g}$, the second involution law and the associative law for relative multiplication, Lemma 3.3 (with $y$ in place of $x$ ), Lemma 2.3(iii), and the left-regularity of $a$ to arrive at

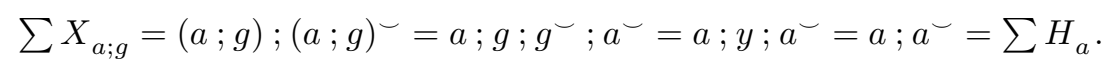

Since $X_{a ; g}$ and $H_{a}$ are sets of atoms, we may conclude from this computation that

$$
X_{a ; g}=H_{a} .
$$

Use Lemmas 3.3 and 2.3(iii) to obtain

$$
\begin{array}{rll}
f ; a ; g=a ; g \quad \text { if and only if } & f ; a ; g ; g^{\smile}=a ; g ; g^{\smile}, \\
& \text { if and only if } \quad f ; a ; y=a ; y, \\
& \text { if and only if } \quad f ; a=a .
\end{array}
$$

These equivalences show that $f$ belongs to the left stabilizer $H_{a ; g}$ if and only if it belongs to the left stabilizer $H_{a}$, so that

$$
H_{a ; g}=H_{a} .
$$

Combine (3) amd (4) to arrive at

$$
X_{a ; g}=H_{a ; g}=H_{a},
$$

and use these equalities together with Corollary 4.3 (with $a ; g$ in place of $a$ ) to conclude that $a ; g$ is left-regular and its left stabilizer is $H_{a}$.

The following corollary is a very important consequence of Atomic Partition Lemma 4.11 and Translation Lemma 4.15.

Corollary 4.16. Let $x$ and $y$ be measurable atoms. If $a \leq x ; 1 ; y$ is an atom, then its left and right stabilizers $H_{a}$ and $K_{a}$ are normal subgroups of $G_{x}$ and $G_{y}$ respectively. If $b \leq x ; 1 ; y$ is also an atom, then $H_{b}=H_{a}$ and $K_{b}=K_{a}$.

Proof. Let $a$ and $b$ be arbitrary atoms below $x ; 1 ; y$. The version of Lemma 4.11 for right-regular elements says that the right translations of $a$ constitute all of the atoms below $x ; 1 ; y$. In particular, there must be an element $g$ in $G_{y}$ such that $b=a ; g$. Use this equality and part (ii) of Lemma 4.15 to obtain

$$
H_{b}=H_{a ; g}=H_{a} .
$$

For any element $f$ in $G_{x}$, the left translation $f ; a$ is an atom below $x ; 1 ; y$, by Lemma 4.11. Take this element for $b$ in (1), and use part (i) of Lemma 4.15 to arrive at

$$
H_{a}=H_{f ; a}=f ; H_{a} ; f^{\smile} .
$$

The equality of the first and last terms in (2) for every $f$ in $G_{x}$ implies that the subgroup $H_{a}$ is normal in $G_{x}$, and (1) implies that every atom $b \leq x ; 1 ; y$ has the 
same left stabilizer as the atom $a$. A dual argument yields the corresponding result for the right stabilizer $K_{a}$.

If a left-regular element $a \leq x ; 1 ; y$ has a normal left stabilizer, then part (i) of Translation Lemma 4.15 implies that any left translation of $a$ is a left-regular element with the same left stabilizer as $a$. The next lemma implies that any leftregular element below $x ; 1 ; y$ with the same left stabilizer as $a$ must in fact be a left translation of $a$. Thus, the left translations of $a$ are precisely the left-regular elements below $x ; 1 ; y$ with the same left stabilizer as $a$. In fact, this property characterizes left-regular elements with normal left-stabilizers.

Lemma 4.17 (Second Translation Lemma). Let $x$ and $y$ be measurable atoms. For every left-regular element $a \leq x ; 1 ; y$, the following assertions are equivalent.

(i) $H_{a}$ is a normal subgroup of $G_{x}$.

(ii) For every left-regular element $b \leq x ; 1 ; y$, we have $H_{b} \subseteq H_{a}$ if and only if $b$ is below some left translation of $a$.

(iii) For every left-regular element $b \leq x ; 1 ; y$, we have $H_{a} \subseteq H_{b}$ if and only if $b$ is above some left translation of $a$.

(iv) For every left-regular element $b \leq x ; 1 ; y$, we have $H_{b}=H_{a}$ if and only if $b$ is equal to some left translation of $a$.

Proof. Assume $a \leq x ; 1 ; y$ is left-regular. For each element $f$ in $G_{x}$, the left translation $f ; a$ is left-regular, and

$$
H_{f ; a}=f ; H_{a} ; f^{\smile},
$$

by part (i) of Translation Lemma 4.15 .

To establish the implication from (i) to each of (ii), (iii), and (iv), assume that the left stabilizer $H_{a}$ is a normal subgroup, and use (1) to obtain

$$
H_{f ; a}=H_{a} \text {. }
$$

Consider any left-regular element $b \leq x ; 1 ; y$. Partition Lemma 4.9 implies that

$$
x ; 1 ; y=\sum\left\{f ; a: f \in G_{x}\right\},
$$

and $b$ is non-zero, by Lemma 4.4 , so

$$
b \cdot(f ; a) \neq 0
$$

for some $f$ in $G_{x}$. Use (2), and then use Lemma 4.14 (with $b$ and $f ; a$ in place of $a$ and $b$ respectively) and (3), to arrive at

$$
\begin{aligned}
H_{b} \subseteq H_{a} & \text { if and only if } & H_{b} \subseteq H_{f ; a}, \\
& \text { if and only if } & b \leq f ; a .
\end{aligned}
$$

A similar argument yields

$$
\begin{array}{rlr}
H_{a} \subseteq H_{b} & \text { if and only if } \quad H_{f ; a} \subseteq H_{b}, \\
& \text { if and only if } \quad f ; a \leq b .
\end{array}
$$

Combine these equivalences to conclude that

$$
H_{b}=H_{a} \quad \text { if and only if } \quad f ; a=b .
$$


To establish the implication from (ii) to (i), assume that (ii) holds, and consider an arbitrary element $f$ in $G_{x}$. The element $b=f ; a$ is left-regular, by the initial observation of this proof, and obviously $b \leq f ; a$, so (ii) implies that

$$
H_{b} \subseteq H_{a} .
$$

Use (1), the choice of $b$, and (4) to see that

$$
f ; H_{a} ; f^{\smile}=H_{f ; a}=H_{b} \subseteq H_{a} .
$$

The inclusion of the left side of (5) in the right side holds for all $f$ in $G_{x}$, so $H_{a}$ must be a normal subgroup of $G_{x}$.

The proof of the implication from (iii) to (i) is similar to the preceding argument, but uses the fact that the subgroup $H_{a}$ is normal just in case

$$
H_{a} \subseteq f ; H_{a} ; f^{\smile}
$$

for every element $f$ in $G_{x}$. The implication from (iv) to (i) is a consequence of the implication from (ii) to (i).

Product Lemma 4.13 has as a hypothesis that the product of the two left-regular elements $a$ and $b$ be non-zero. The next lemma gives necessary and sufficient conditions for this hypothesis to be satisfied, under the additional assumption that the left stabilizers are normal. It also characterizes the product subgroup $H_{a} ; H_{b}$ as the left stabilizer of a specific element. Recall from Lemma 4.13 that

$$
H_{a \cdot b}=H_{a} \cap H_{b},
$$

so that the coset system for $H_{a \cdot b}$ coincides with the coset system for $H_{a} \cap H_{b}$, which is

$$
\left\langle H_{a, \xi} \cap H_{b, \eta}: \xi<\kappa \text { and } \eta<\lambda\right\rangle,
$$

where

$$
\left\langle H_{a, \xi}: \xi<\kappa\right\rangle \quad \text { and } \quad\left\langle H_{b, \eta}: \eta<\lambda\right\rangle
$$

are respectively coset systems for $H_{a}$ and $H_{b}$ in $H_{a} ; H_{b}$.

Lemma 4.18 (Second Product Lemma). Let $x$ and $y$ be measurable atoms, and a and $b$ left-regular elements below $x ; 1 ; y$ with normal stabilizers $H_{a}$ and $H_{b}$.

(i) $a \cdot b \neq 0$ if and only if $a ; a^{\smile} ; b=b ; b^{\smile} ; a$.

(ii) If $a \cdot b \neq 0$, then the product subgroup $H_{a} ; H_{b}$ is the left stabilizer of the element $b ; b^{-} ; a$, and the system of left translations

$$
\left\langle\left(H_{a, \xi} \cap H_{b, \eta}\right) ;(a \cdot b): \xi<\kappa \text { and } \eta<\lambda\right\rangle
$$

is a partition of $b ; b^{-} ; a$, where $\left\langle H_{a, \xi}: \xi<\kappa\right\rangle$ and $\left\langle H_{b, \eta}: \eta<\lambda\right\rangle$ are cosets systems for $H_{a}$ and $H_{b}$ in $H_{a} ; H_{b}$. Different left translations of $a \cdot b$ coincide with the different products of the left translations of $a$ and $b$ in the sense that

$$
\left(H_{a, \xi} \cap H_{b, \eta}\right) ;(a \cdot b)=\left(H_{a, \xi} ; a\right) \cdot\left(H_{b, \eta} ; b\right)
$$

for every $\xi<\kappa$ and $\eta<\lambda$.

Proof. To prove (i), assume first that $a \cdot b \neq 0$. The product $a \cdot b$ is then a left regular element, by Product Lemma 4.13. Consequently,

$$
a ; a^{\smile} ;(a \cdot b)=\left(\sum H_{a}\right) ;(a \cdot b)=\sum H_{a} ;(a \cdot b)=H_{a} ; a=a
$$

by the assumed left-regularity of $a$, complete distributivity, the final assertion of Partition Lemma 4.12 (with $a \cdot b$ and $a$ in place of $a$ and $b$ respectively, and $H_{a}$ in 
place of $H_{b, \eta}$ ), and the fact that $H_{a}$ is the stabilizer of $a$. Use (1) and monotony to get

$$
a \leq a ; a^{\smile} ; b .
$$

Form the relative product of both sides of this inequality on the left with $b ; b^{-}$, and then use monotony, the definition of a regular element and the assumed leftregularity of $a$ and $b$, complete distributivity, the assumption that $H_{a}$ is normal, the definition of $H_{b}$ as the stabilizer of $b$, complete distributivity, and the left-regularity of $a$, to arrive at

$$
\begin{aligned}
b ; b^{\smile} ; a \leq b ; b^{\smile} ; a ; a^{\smile} ; b=\left(\sum H_{b}\right) ;\left(\sum H_{a}\right) ; b=\sum H_{b} ; H_{a} ; b \\
\quad=\sum H_{a} ; H_{b} ; b=\sum H_{a} ; b=\left(\sum H_{a}\right) ; b=a ; a^{\smile} ; b .
\end{aligned}
$$

A symmetric argument yields the reverse inequality. This establishes the implication from left to right in part (i).

To establish the reverse implication, assume that

$$
a ; a^{\smile} ; b=b ; b^{\smile} ; a .
$$

Use the definition of a left-regular element and the assumed left-regularity of $b$, complete distributivity, the definition of $H_{a}$ as the stabilizer of $a$, and the assumption that this stabilizer is a normal subgroup to get

$$
b ; b^{\smile} ; a=\left(\sum H_{b}\right) ; a=\sum H_{b} ; a=\sum H_{b} ; H_{a} ; a=\sum H_{a} ; H_{b} ; a .
$$

The product subgroup $H_{a} ; H_{b}$ is the union of the cosets $H_{a, \xi}$ of $H_{a}$ in $H_{a} ; H_{b}$, by assumption, so

$$
\sum H_{a} ; H_{b} ; a=\sum\left(\bigcup_{\xi} H_{a, \xi}\right) ; a=\sum_{\xi} H_{a, \xi} ; a,
$$

by complete distributivity. Also, the elements $H_{a, \xi} ; a$ are non-zero and pairwise disjoint, by Partition Lemma 4.9. Combine this observation with (3) and (4) to see that

$$
\left\langle H_{a, \xi} ; a: \xi<\kappa\right\rangle
$$

is a partition of $b ; b^{\smile} ; a$. A similar argument shows that

$$
\left\langle H_{b, \eta} ; b: \eta<\lambda\right\rangle
$$

is a partition of $a ; a^{\smile} ; b$.

Use (2), Lemma 2.3(i), monotony, Lemma 2.3(iii), the left-regularity of $b$, and Lemma 4.4 to obtain

$$
b ; b^{\smile} ; a=a ; a^{\smile} ; b \geq x ; b=b>0 .
$$

Since (5) is a partition of $b ; b^{\smile} ; a$, it follows from (7) that there must be an index $\gamma<\kappa$ such that

$$
\left(H_{a, \gamma} ; a\right) \cdot b \neq 0 .
$$

Put $\bar{a}=H_{a, \gamma} ; a$ and write the preceding inequality as

$$
\bar{a} \cdot b \neq 0 .
$$

The element $\bar{a}$ is, by definition, a left translation of the left-regular element $a$ with a normal left stabilizer, so $\bar{a}$ is itself left-regular with the same normal left stabilizer $H_{a}$, by part (i) of Translation Lemma 4.15. In view of (8), Product Lemma 4.13 may be applied (with $\bar{a}$ in place of $a$ ) to conclude that $\bar{a} \cdot b$ is left-regular with left stabilizer $H_{a} \cap H_{b}$. 
As is well known from group theory, the normal subgroup $H_{a} \cap H_{b}$ has the coset system

$$
\left\langle H_{a, \xi} \cap H_{b, \eta}: \xi<\kappa \text { and } \eta<\lambda\right\rangle
$$

in $H_{a} ; H_{b}$. Every left translation of a left-regular element by a left coset of its left stabilizer is again left-regular, by part (i) of Translation Lemma 4.15. In particular, each left translation

$$
\left(H_{a, \xi} \cap H_{b, \eta}\right) ;(\bar{a} \cdot b)
$$

of $\bar{a} \cdot b$ is left-regular, and therefore non-zero, by Lemma 4.4. Choose $\xi$ so that $H_{a, \xi}$ is the coset inverse of $H_{a, \gamma}$ in the quotient group $G_{x} / H_{a}$, and use monotony, the definition of $\bar{a}$, the inverse property from group theory, and the definition of $H_{a}$ as the left stabilizer of $a$ to obtain

$$
\left(H_{a, \xi} \cap H_{b, \eta}\right) ;(\bar{a} \cdot b) \leq H_{a, \xi} ; \bar{a}=H_{a, \xi} ; H_{a, \gamma} ; a=H_{a} ; a=a .
$$

Similarly, take $\eta=0$, so that $H_{b, \eta}$ coincides with the identity coset $H_{b}$, and use monotony and the definition of $H_{b}$ to obtain

$$
\left(H_{a, \xi} \cap H_{b, \eta}\right) ;(\bar{a} \cdot b) \leq H_{b, \eta} ; b=H_{b} ; b=b .
$$

Form the products of the left and right sides of (10) and (11), and use Boolean algebra to arrive at

$$
0<\left(H_{a, \xi} \cap H_{b, \eta}\right) ;(\bar{a} \cdot b) \leq a \cdot b .
$$

Conclusion: $a \cdot b \neq 0$, as was to be shown.

To prove (ii), assume that $a \cdot b \neq 0$. It follows from part (i) of the lemma that (2) also holds, so it makes sense to write

$$
c=b ; b^{\smile} ; a=a ; a^{\smile} ; b .
$$

The first task is to check that the product subgroup $H_{a} ; H_{b}$ coincides with the left stabilizer $H_{c}$. Consider an element $h$ in $G_{x}$. If $h$ is in the product subgroup, then there must be elements $f$ in $H_{a}$ and $g$ in $H_{b}$ such that $h=f ; g$, by the definition of the product subgroup. Consequently,

$$
\begin{aligned}
h ; c=h ; b ; b^{\smile} ; a=f ; g ; b ; b^{\smile} ; a=f ; b ; b^{\smile} ; a \\
\quad=f ; a ; a^{-} ; b=a ; a^{-} ; b=b ; b^{-} ; a=c,
\end{aligned}
$$

by (12), the assumptions on $h$, the assumption that $g$ is in the left stabilizer of $b$, part (i) of the lemma, which implies that (2) holds, the assumption that $f$ is in the left stabilizer of $a$, and (2) again. It follows from (13) that $h$ belongs to the left stabilizer $H_{c}$.

On the other hand, if $h$ belongs to $H_{c}$, then

$$
h ; b ; b^{-} ; a=h ; c=c=b ; b^{-} ; a,
$$

by (12) and the definition of $H_{c}$. Form the relative product of the left and right sides of (14), on the right, with $a^{\smile}$ to obtain

$$
h ; b ; b^{\smile} ; a ; a^{\smile}=b ; b^{\smile} ; a ; a^{\smile} .
$$

Use complete distributivity, the left-regularity of $a$ and $b$, (15), the left-regularity of $a$ and $b$ again, and complete distributivity to get

$$
\begin{aligned}
\sum H_{b} ; H_{a}=\left(\sum H_{b}\right) ;\left(\sum H_{a}\right)=b ; b^{\smile} ; a ; a^{\smile}=h ; b ; b^{\smile} ; a ; a^{\smile} \\
=h ;\left(\sum H_{b}\right) ;\left(\sum H_{a}\right)=h ;\left(\sum H_{b} ; H_{a}\right)=\sum h ; H_{b} ; H_{a} .
\end{aligned}
$$


The sets involved in the first and last sums of (16) are sets of atoms, so the two sets must be equal. Combine this with the assumption that $H_{a}$ is normal to arrive at

$$
H_{a} ; H_{b}=H_{b} ; H_{a}=h ; H_{b} ; H_{a}=h ; H_{a} ; H_{b} .
$$

Thus, the left coset of the product subgroup $H_{a} ; H_{b}$ determined by each element $h$ belonging to the left stabilizer $H_{c}$ coincides with $H_{a} ; H_{b}$, so each such $h$ belongs to the product subgroup. Conclusion:

$$
H_{c}=H_{a} ; H_{b} .
$$

Product Lemma 4.13, and the assumption that $a$ and $b$ are left-regular elements with $a \cdot b \neq 0$ imply that $a \cdot b$ is a left-regular element with left stabilizer $H_{a} \cap H_{b}$. Recall that (9) is a coset system for this left stabilizer in $H_{a} ; H_{b}$. Consequently, $H_{c}$ is the union of the cosets $H_{a, \xi} \cap H_{b, \eta}$ for $\xi<\kappa$ and $\eta<\lambda$, by (17). The assumption that $a \cdot b \neq 0$, together with Boolean algebra, (7), and (12), implies that

$$
0<a \cdot b \leq b \leq c,
$$

In view of these observations, Partition Lemma 4.12 may be applied (with $a \cdot b$ and $c$ in place of $a$ and $b$ respectively, and with $H_{a, \xi} \cap H_{b, \eta}$ and $H_{c}$ in place of $H_{a, \xi}$ and $H_{b, \eta}$ respectively) to conclude that

$$
\left\langle\left(H_{a, \xi} \cap H_{b, \eta}\right) ;(a \cdot b): \xi<\kappa \text { and } \eta<\lambda\right\rangle
$$

is a partition of $H_{c} ; c$, and therefore a partition of $c$, by the definition of $H_{c}$ as the left stabilizer of $c$. In particular, the elements in this system are non-zero and pairwise disjoint.

Monotony implies that

$$
\left(H_{a, \xi} \cap H_{b, \eta}\right) ;(a \cdot b) \leq H_{a, \xi} ; a \quad \text { and } \quad\left(H_{a, \xi} \cap H_{b, \eta}\right) ;(a \cdot b) \leq H_{b, \eta} ; b,
$$

and therefore

$$
\left(H_{a, \xi} \cap H_{b, \eta}\right) ;(a \cdot b) \leq\left(H_{a, \xi} ; a\right) \cdot\left(H_{b, \eta} ; b\right),
$$

by Boolean algebra. The elements on the left side of this inequality are left-regular and therefore non-zero, so the products on the right must also be non-zero. It has already been shown that, on the basis of (2), the systems of left translations in (5) and in (6) are both partitions of $c$. Combine this with the preceding observation, and use Boolean algebra, to conclude that the system

$$
\left\langle\left(H_{a, \xi} ; a\right) \cdot\left(H_{b, \eta} ; b\right): \xi<\kappa \text { and } \eta<\lambda\right\rangle
$$

is also a partition of $c$. Summarizing, (18) and (20) are both partitions of $c$. The inequality in (19) therefore implies that the two partitions must coincide, so that equality holds in (19). This completes the proof of (ii).

\section{Regular elements With noRmal stabilizers}

Suppose $x$ and $y$ are measurable atoms, and $a \leq x ; 1 ; y$ a regular element with normal stabilizers. The assumption on the stabilizers implies, in particular, that it is possible to form the quotient groups $G_{x} / H_{a}$ and $G_{y} / K_{a}$. It turns out that the element $a$ induces in a canonical fashion an isomorphism between these two quotients. To prove this, we begin with a lemma. 
Lemma 5.1. Let $x$ and $y$ be measurable atoms. If an element $a \leq x ; 1 ; y$ is regular with normal stabilizers, then for every $f$ in $G_{x}$ and every $g$ in $G_{y}$,

$$
(f ; a) \cdot(a ; g) \neq 0 \quad \text { if and only if } \quad f ; a=a ; g .
$$

Proof. The implication from right to left is obvious, since translations of regular elements are regular, by Translation Lemma 4.15, and regular elements are never zero, by Lemma 4.4. To derive the reverse implication, assume that the hypotheses of the lemma are satisfied, and suppose that

$$
(f ; a) \cdot(a ; g) \neq 0 .
$$

Both $f ; a$ and $a ; g$ are regular elements below $x ; 1 ; y$, by Translation Lemma 4.15 and its right-regular version, so the product

$$
c=(f ; a) \cdot(a ; g)
$$

must be a regular element below $x ; 1 ; y$, by the Product Lemma 4.13 and its rightregular version. Moreover, this product has the same left and right stabilizers as $a$, because

$$
H_{c}=H_{f ; a} \cap H_{a ; g}=\left(f ; H_{a} ; f^{\sim}\right) \cap H_{a}=H_{a},
$$

by (2), Product Lemma 4.13, Translation Lemma 4.15, and the assumption that $H_{a}$ is normal, and similarly,

$$
K_{c}=K_{f ; a} \cap K_{a ; g}=K_{a} \cap\left(g^{\smile} ; K_{a} ; g\right)=K_{a},
$$

by the right-regular versions of Lemmas 4.13 and 4.15 .

Use the assumption that $H_{a}$ is normal, the regularity of $c,(3)$, and the implication from (i) to (iv) in Translation Lemma 4.17 (with $c$ in place of $b$ ) to get that $c$ is a left translation of $a$, in symbols,

$$
c=(f ; a) \cdot(a ; g)=h ; a
$$

for some $h$ in $G_{x}$. In particular, $h ; a \leq f ; a$. Any two left translations of $a$ are equal or disjoint, by Lemma 4.6(iii), so $h ; a=f ; a$. It follows from this equation, (4), and Boolean algebra that $f ; a \leq a ; g$. A dual argument using right-regularity establishes the reverse inequality.

Corollary 5.2. Let $x$ and $y$ be measurable atoms. If $a \leq x ; 1 ; y$ is a regular element with normal stabilizers, then every right translation of a is also a left translation, and conversely.

Proof. If an element $a$ satisfies the hypotheses of the corollary, then $a$ and all of its translations are regular elements below $x ; 1 ; y$, by Translation Lemma 4.15 and its right-regular version, and in particular they are not 0 , by Lemma 4.4. Let $g$ be any element in $G_{y}$. Since the left translations $f ; a$, for $f$ in $G_{x}$, sum to $x ; 1 ; y$, by Partition Lemma 4.9, there must be an element $f$ in $G_{x}$ such that

$$
(f ; a) \cdot(a ; g) \neq 0 .
$$

Consequently,

$$
(f ; a)=(a ; g),
$$

by Lemma 5.1. In other words, the right translation $a ; g$ can be written as a left translation $f ; a$. The converse is proved in a similar way. 
We turn now to the task of constructing a group triple in an atomic, measurable relation algebra, and verifying the semi-frame conditions for this triple. The definitions of these notions, which are from [1], are not needed in this section, but they are needed in the next section. We give them here in order to motivate the discussion. The reader may choose to ignore them for now and refer back to them at the appropriate moment in the next section. A group triple

$$
\mathcal{F}=(G, \varphi, C)
$$

consists of a system

$$
G=\left\langle G_{x}: x \in I\right\rangle
$$

of disjoint groups, a system

$$
\varphi=\left\langle\varphi_{x y}:(x, y) \in \mathcal{E}\right\rangle
$$

of associated quotient isomorphisms, with $\varphi_{x y}$ mapping a quotient group $G_{x} / H_{x y}$ to a quotient group $G_{y} / K_{x y}$ for each pair $(x, y)$ in a fixed equivalence relation $\mathcal{E}$ on the group index set $I$, and finally a system

$$
C=\left\langle C_{x y z}:(x, y, z) \in \mathcal{E}_{3}\right\rangle
$$

of associated cosets, with $C_{x y z}$ a coset of the normal subgroup $H_{x y}{ }^{\circ} H_{x z}$ in $G_{x}$ for each triple $(x, y, z)$ in the set $\mathcal{E}_{3}$ of triples $(x, y, z)$ such that $(x, y)$ and $(y, z)$ are both in $\mathcal{E}$.

Definition 5.3. A group triple

$$
\mathcal{F}=(G, \varphi, C)
$$

is a (coset) semi-frame if the following semi-frame conditions are satisfied.

(i) $\varphi_{x x}$ is the identity automorphism of $G_{x} /\left\{e_{x}\right\}$ for all $x$ in $I$.

(ii) $\varphi_{y x}=\varphi_{x y}^{-1}$ whenever $(x, y)$ is in $\mathcal{E}$.

(iii) $\varphi_{x y}\left[H_{x y} \circ H_{x z}\right]=K_{x y} \circ H_{y z}$ whenever $(x, y, z)$ is in $\mathcal{E}_{3}$.

(iv) $\hat{\varphi}_{x y}\left|\hat{\varphi}_{y z}=\tau\right| \hat{\varphi}_{x z}$ whenever $(x, y, z)$ is in $\mathcal{E}_{3}$,

where $\hat{\varphi}_{x y}, \hat{\varphi}_{y z}$, and $\hat{\varphi}_{x z}$ are the quotient isomorphisms induced on

$$
G_{x} /\left(H_{x y} \circ H_{x z}\right), \quad G_{y} /\left(K_{x y} \circ H_{y z}\right), G_{x} /\left(H_{x y} \circ H_{x z}\right)
$$

by $\varphi_{x y}, \varphi_{y z}, \varphi_{x z}$ respectively, and $\tau$ is the inner automorphism of $G_{x} /\left(H_{x y} \circ H_{x z}\right)$ determined by the coset $C_{x y z}$.

The global assumption of measurability is not needed in order to accomplish most of the task of constructing a group triple and verifying the semi-frame conditions, so for now we continue with the local assumptions that $x$ and $y$ are measurable atoms, and $a \leq x ; 1 ; y$ is a regular element with normal stabilizers.

Theorem 5.4 (First Isomorphism Theorem). Let $x$ and $y$ be measurable atoms. If $a \leq x ; 1 ; y$ is a regular element with normal stabilizers, and in particular, if $a$ is an atom, then the quotient groups $G_{x} / H_{a}$ and $G_{y} / K_{a}$ are isomorphic. In fact, if

$$
\left\langle H_{\xi}: \xi<\kappa\right\rangle \quad \text { and } \quad\left\langle K_{\eta}: \eta<\lambda\right\rangle
$$

are coset systems of $H_{a}$ in $G_{x}$ and $K_{a}$ in $G_{y}$ respectively, then there is a unique bijection $\varphi$ from $\kappa$ to $\lambda$ such that

$$
H_{\xi} ; a=a ; K_{\varphi(\xi)},
$$

and the mapping $H_{\xi} \longmapsto K_{\varphi(\xi)}$ is the desired isomorphism. 
Proof. Consider a regular element $a \leq x ; 1 ; y$ with normal stabilizers. The left and right translations of $a$ are just the elements in the systems

$$
\left\langle H_{\xi} ; a: \xi<\kappa\right\rangle \quad \text { and } \quad\left\langle a ; K_{\eta}: \eta<\lambda\right\rangle
$$

respectively. For any fixed $\xi<\kappa$, there is a $\xi^{\prime}<\lambda$ such that

$$
H_{\xi} ; a=a ; K_{\xi^{\prime}} \text {, }
$$

by Corollary 5.2. If $\xi^{\prime \prime}<\lambda$ is any other index such that

$$
H_{\xi} ; a=a ; K_{\xi^{\prime \prime}}
$$

then

$$
a ; K_{\xi^{\prime}}=a ; K_{\xi^{\prime \prime}}
$$

and therefore $\xi^{\prime}=\xi^{\prime \prime}$, because distinct cosets of $K_{a}$ lead to disjoint right translations of $a$, by the right-regular version of Lemma 4.6(iii). Thus, there is a unique $\xi^{\prime}<\lambda$ such that (1) holds. Define $\varphi(\xi)$ to be $\xi^{\prime}$. The preceding remarks imply that $\varphi$ is a well-defined mapping from $\kappa$ into $\lambda$, and

$$
H_{\xi} ; a=a ; K_{\varphi(\xi)}
$$

for each $\xi<\kappa$, by (1) and the definition of $\varphi$.

In a completely analogous fashion, define a mapping $\psi$ from $\lambda$ into $\kappa$ such that

$$
a ; K_{\eta}=H_{\psi(\eta)} ; a
$$

for each $\eta<\lambda$. In particular,

$$
H_{\xi} ; a=a ; K_{\varphi(\xi)}=H_{\psi(\varphi(\xi))} ; a,
$$

by (2) and (3) (with $\varphi(\xi)$ in place of $\eta$ ). Distinct cosets of $H_{a}$ lead to disjoint left translations of $a$, by Partiton Lemma 4.9, so $\psi(\varphi(\xi))=\xi$ for each $\xi<\kappa$, by (4). A symmetric argument shows that $\varphi(\psi(\eta))=\eta$ for each $\eta<\lambda$. It follows that $\varphi$ must a bijection from $\kappa$ to $\lambda$, with $\psi$ as its inverse. Consequently, the correspondence

$$
H_{\xi} \longmapsto K_{\varphi(\xi)}
$$

is a bijection from $G_{x} / H_{a}$ to $G_{y} / K_{a}$.

Suppose that

$$
H_{\xi} ; H_{\eta}=H_{\mu} .
$$

Use (2), (6), and two more applications of (2) to arrive at

$$
a ; K_{\varphi(\mu)}=H_{\mu} ; a=H_{\xi} ; H_{\eta} ; a=H_{\xi} ; a ; K_{\varphi(\eta)}=a ; K_{\varphi(\xi)} ; K_{\varphi(\eta)} .
$$

Distinct cosets of $K_{a}$ lead to distinct right translations of $a$, by the right-regular version of Lemma 4.9, so (7) implies that

$$
K_{\varphi(\xi)} ; K_{\varphi(\eta)}=K_{\varphi(\mu)},
$$

This argument shows that the mapping (5) preserves the quotient group composition operation of forming the relative product of two cosets.

In groups, the identity element and the operation of forming inverses are both definable in terms of the group composition operation, so any bijection that preserves composition is automatically a group isomorphism. In particular, it follows from the preceding observations that the mapping (5) must be an isomorphism between the quotient groups. 
Convention 5.5. It is clear from the First Isomorphism Theorem that we may assume $\kappa=\lambda$, and we may reindex the coset system $\left\langle K_{\xi}: \xi<\kappa\right\rangle$ so that $\varphi(\xi)=\xi$, that is to say, so that

$$
H_{\xi} \longmapsto K_{\xi}
$$

is the canonical isomorphism from $G_{x} / H_{a}$ to $G_{y} / K_{a}$ determined by the equation

$$
H_{\xi} ; a=a ; K_{\xi} \text {. }
$$

Furthermore, it may be supposed that $H_{0}=H_{a}$, and consequently that $K_{0}=K_{a}$ (where 0 denotes an index zero, and not the zero element in the relation algebra $\mathfrak{A}$ that has been fixed throughout the discussion.) In the subsequent development, these notational conventions shall be adopted. When an explicit reference to $a$ is required, we write $H_{a, \xi}$ and $K_{a, \xi}$ for $H_{\xi}$ and $K_{\xi}$ respectively, and we denote the canonical isomorphism (1) by $\varphi_{a}$, and call it the isomorphism determined by a.

The particular isomorphism described in Isomorphism Theorem 5.4 is determined by the element $a$. Different translations of $a$ may determine different isomorphisms. How are these various isomorphisms related to one other? Assume that $b$ is, for example, a left translation of $a$, say $b=H_{\eta} ; a$. In this case, $b$ is also a regular element with the same normal stabilizer as $a$, by Translation Lemma 4.15 and its right-regular version. Moreover, if $\varphi_{a}$ maps $H_{\xi}$ to $K_{\xi}$ for each $\xi<\kappa$, as in Convention 5.5, or in different words, if equation (2) of the convention holds for each $\xi<\kappa$, then

$$
b=H_{\eta} ; a=a ; K_{\eta},
$$

and therefore

$$
\begin{aligned}
H_{\xi} ; b=H_{\xi} ; H_{\eta} ; a=H_{\xi} ; a ; K_{\eta} & =a ; K_{\xi} ; K_{\eta} \\
& =a ; K_{\eta} ; K_{\eta}^{\smile} ; K_{\xi} ; K_{\eta}=b ; K_{\eta}^{\smile} ; K_{\xi} ; K_{\eta},
\end{aligned}
$$

by (3) and (2). The fourth equality uses quotient group identity and inverse properties:

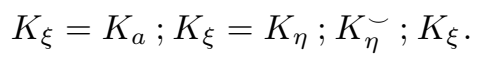

It follows from (4) and the definition of the isomorphism determined by $b$ that

$$
\varphi_{b}\left(H_{\xi}\right)=K_{\eta}^{\smile} ; K_{\xi} ; K_{\eta}
$$

for each $\xi$.

If $\tau$ is the inner automorphism of $G_{x} / H_{a}$ determined by

$$
\tau\left(H_{\xi}\right)=H_{\eta}^{\smile} ; H_{\xi} ; H_{\eta}
$$

for each $\xi$, then

$$
\varphi_{b}=\tau \mid \varphi_{a} .
$$

The verification of (7) is an easy computation:

$$
\begin{array}{r}
\left(\tau \mid \varphi_{a}\right)\left(H_{\xi}\right)=\varphi_{a}\left(\tau\left(H_{\xi}\right)\right)=\varphi_{a}\left(H_{\eta}^{\smile} ; H_{\xi} ; H_{\eta}\right)=\varphi_{a}\left(H_{\eta}\right)^{\smile} ; \varphi_{a}\left(H_{\xi}\right) ; \varphi_{a}\left(H_{\eta}\right) \\
=K_{\eta}^{\smile} ; K_{\xi} ; K_{\eta}=\varphi_{b}\left(H_{\xi}\right),
\end{array}
$$

by the definition of relational composition, (6), the isomorphism properties of $\varphi_{a}$, the definition of $\varphi_{a}$, and (5). The isomorphism $\varphi_{b}$ agrees with the isomorphism $\varphi_{a}$ 
on a coset $H_{\xi}$ just in case the images of $H_{\xi}$ under these two mappings are equal, that is to say, just in case

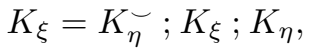

or, equivalently, just in case

$$
H_{\xi}=H_{\eta}^{\smile} ; H_{\xi} ; H_{\eta} \text {. }
$$

This is the same as saying that $H_{\eta}$ commutes with $H_{\xi}$.

The preceding argument proves the following theorem.

Theorem 5.6 (Second Isomorphism Theorem). Let $x$ and $y$ be measurable atoms, and $a \leq x ; 1 ; y$ a regular element with normal stabilizers. If $b$ is the left translation of a by a coset $H_{\eta}$ of $H_{a}$, then $\varphi_{b}$ is just the relational composition

$$
\varphi_{b}=\tau \mid \varphi_{a}
$$

where $\tau$ is the inner automorphism of $G_{x} / H_{a}$ determined by

$$
\tau\left(H_{\xi}\right)=H_{\eta}^{\smile} ; H_{\xi} ; H_{\eta} .
$$

The isomorphism $\varphi_{b}$ agrees with $\varphi_{a}$ on precisely those cosets of $H_{a}$ that commute with $H_{\eta}$.

The theorem allows us to characterize when the isomorphisms determined by a left translation $b$ of a regular element $a$ coincides with the isomorphism $\varphi_{a}$.

Corollary 5.7. Under the hypotheses of the First Isomorphism Theorem, if $b$ is the left translation of a by a coset $H_{\eta}$ of $H_{a}$, then

$$
\varphi_{b}=\varphi_{a}
$$

just in case $H_{\eta}$ is in the center of $G_{x} / H_{a}$.

Corollary 5.8. Under the hypotheses of the First Isomorphism Theorem, if $G_{x} / H_{a}$ is abelian, then $\varphi_{b}=\varphi_{a}$ for every translation $b$ of $a$.

We now explore some of the important consequences of the Isomorphism Theorems. Let $x$ and $y$ be measurable atoms. A regular element $a \leq x ; 1 ; y$ with normal stabilizers determines an isomorphism $\varphi_{a}$ from $G_{x} / H_{a}$ to $G_{y} / K_{a}$, by Isomorphism Theorem 5.4. Another regular element $b \leq x ; 1 ; y$ with normal stabilizers (with $b$ not necessarily a translation of $a$ ) determines an isomorphism $\varphi_{b}$ from $G_{x} / H_{b}$ to $G_{y} / K_{b}$. What is the relationship between these two isomorphisms when $a \leq b$ ? To answer this question, we use the notation and conventions discussed in Convention 5.5. In particular,

$$
\left\langle H_{a, \xi}: \xi<\kappa\right\rangle \quad \text { and } \quad\left\langle H_{b, \eta}: \eta<\lambda\right\rangle
$$

are assumed to be coset systems for $H_{a}$ and $H_{b}$ respectively in $G_{x}$, and

$$
\left\langle K_{a, \xi}: \xi<\kappa\right\rangle \quad \text { and } \quad\left\langle K_{b, \eta}: \eta<\lambda\right\rangle
$$

are assumed to be the corresponding coset systems for $K_{a}$ and $K_{b}$ respectively in $G_{y}$.

If $a \leq b$, then

$$
H_{a} \subseteq H_{b} \quad \text { and } \quad K_{a} \subseteq K_{b},
$$


by Lemma 4.8 and its right-regular version, so there are partitions $\left\langle\Gamma_{\eta}: \eta<\lambda\right\rangle$ and $\left\langle\Delta_{\eta}: \eta<\lambda\right\rangle$ of $\kappa$ such that

$$
H_{b, \eta}=\bigcup\left\{H_{a, \xi}: \xi \in \Gamma_{\eta}\right\} \quad \text { and } \quad K_{b, \eta}=\bigcup\left\{K_{a, \xi}: \xi \in \Delta_{\eta}\right\}
$$

for each $\eta<\lambda$. Apply Partition Lemma 4.12 and its right-regular version to see that

$$
\left\langle H_{a, \xi} ; a: \xi \in \Gamma_{\eta}\right\rangle \quad \text { and } \quad\left\langle a ; K_{a, \xi}: \xi \in \Delta_{\eta}\right\rangle
$$

partition $H_{b, \eta} ; b$ and $b ; K_{b, \eta}$ respectively. For each $\eta<\lambda$ and $\xi<\kappa$,

$$
H_{b, \eta} ; b=b ; K_{b, \eta} \quad \text { and } \quad H_{a, \xi} ; a=a ; K_{a, \xi},
$$

by Convention 5.5. Use the partition properties of (3), together with (4), to obtain

$$
b ; K_{b, \eta}=H_{b, \eta} ; b=\sum\left\{H_{a, \xi} ; a: \xi \in \Gamma_{\eta}\right\}=\sum\left\{a ; K_{a, \xi}: \xi \in \Gamma_{\eta}\right\} .
$$

It follows from this computation that the translations $a ; K_{a, \xi}$ with indices $\xi$ in $\Gamma_{\eta}$ partition $b ; K_{b, \eta}$. Compare this with the second part of (3), and use the fact that the right translations of $a$ by distinct cosets of $K_{a}$ are pairwise disjoint, by the rightregular version of Partition Lemma 4.9, to conclude that $\Gamma_{\eta}=\Delta_{\eta}$. Consequently,

$$
K_{b, \eta}=\bigcup\left\{K_{a, \xi}: \xi \in \Gamma_{\eta}\right\},
$$

by $(2)$.

In view of (1) and (2), the isomorphism $\varphi_{a}$ from $G_{x} / H_{a}$ to $G_{y} / K_{a}$ induces in a natural way an isomorphism $\hat{\varphi}_{a}$ from $G_{x} / H_{b}$ to $G_{y} / K_{b}$ that is defined by

$$
\hat{\varphi}_{a}\left(H_{b, \eta}\right)=\bigcup\left\{\varphi_{a}\left(H_{a, \xi}\right): \xi \in \Gamma_{\eta}\right\}
$$

for each $\eta$. It is easy to check that this induced isomorphism coincides with $\varphi_{b}$, because

$$
\hat{\varphi}_{a}\left(H_{b, \eta}\right)=\bigcup\left\{\varphi_{a}\left(H_{a, \xi}\right): \xi \in \Gamma_{\eta}\right\}=\bigcup\left\{K_{a, \xi}: \xi \in \Gamma_{\eta}\right\}=K_{b, \eta}=\varphi_{b}\left(H_{b, \eta}\right),
$$

by the definition of $\hat{\varphi}_{a}$, the definition of $\varphi_{a}$ and Convention 5.5, (5), and the definition of $\varphi_{b}$ and Convention 5.5 (with $b$ in place of $a$ ). The following theorem has been proved.

Theorem 5.9 (Refinement Theorem). Let $x$ and $y$ be measurable atoms, and a and $b$ regular elements below $x ; 1 ; y$ with normal stabilizers. If $a \leq b$, then $H_{a}$ and $K_{a}$ are subgroups of $H_{b}$ and $K_{b}$ respectively, and the isomorphism $\varphi_{a}$ from $G_{x} / H_{a}$ to $G_{y} / K_{a}$ induces an isomorphism $\hat{\varphi}_{a}$ from $G_{x} / H_{b}$ to $G_{y} / K_{b}$, that coincides with $\varphi_{b}$.

A measurable atom $x$ is certainly a regular element with normal stabilizers, by Corollaries 4.7 and 4.16 (with $y=x$ ). By definition, the left stabilizer $H_{x}$ consists of those elements $f$ in $G_{x}$ such that $f ; x=x$. Since $f ; x=f$ for all $f$ in $G_{x}$, by Lemma 3.3, it follows that

$$
f \in H_{x} \quad \text { if and only if } \quad f=x,
$$

and therefore $H_{x}=\{x\}$. Similarly, $K_{x}=\{x\}$. The cosets of these stabilizers are the singletons $\{f\}$ for $f$ in $G_{x}$. The quotient isomorphism $\varphi_{x}$ maps $G_{x} / H_{x}$ to $G_{x} / K_{x}$, and takes $\{f\}$ to $\{g\}$ if and only if $f ; x=x ; g$, that is to say, if and only if $f=g$, by Lemma 3.3. Thus, $\varphi_{x}$ is the identity automorphism of $G_{x} /\{x\}$. This argument proves the following theorem. 
Theorem 5.10 (Identity Theorem). A measurable atom $x$ is a regular element with left and right stabilizers both equal to the trivial normal subgroup $\{x\}$. The quotient isomorphism $\varphi_{x}$ is the identity automorphism of $G_{x} /\{x\}$.

The next theorem says that if $a \leq x ; 1 ; y$ is a regular element with normal stabilizers, then so is $a^{\smile}$, and $\varphi_{a} \smile$ is just the inverse isomorphism of $\varphi_{a}$.

Theorem 5.11 (Converse Theorem). Let $x$ and $y$ be measurable atoms. If $a$ is a regular element bekiw $x ; 1 ; y$ with normal stabilizers, and in particular, if $a$ is an atom, then $a^{-}$is a regular element below $y ; 1 ; x$ with normal stabilizers

$$
H_{a} \smile=K_{a} \quad \text { and } \quad K_{a^{\smile}}=H_{a},
$$

and the quotient isomorphism $\varphi_{a \smile}=\varphi_{a}^{-1}$.

Proof. Assume the hypotheses of the theorem, write $b=a^{\smile}$, and observe that

$$
b=a^{\smile} \leq(x ; 1 ; y)^{-}=y ; 1 ; x,
$$

by the definition of $b$, the assumption that $a \leq x ; 1 ; y$, monotony, and Lemma 2.5(iii). For each element $f$ in $G_{y}$,

$$
\begin{array}{rll}
f \in H_{b} \quad \text { if and only if } & f ; b=b, \\
\text { if and only if } & f ; a^{\smile}=a^{\smile}, \\
\text { if and only if } & a ; f^{\smile}=a, \\
\text { if and only if } & f^{\smile} \in K_{a}, \\
\text { if and only if } & f \in K_{a},
\end{array}
$$

by the definition of $H_{b}$, the definition of $b$, the two involution laws (R6) and (R7), the definition of $K_{a}$, and the fact that $K_{a}$ is a subgroup of $G_{y}$ and therefore closed under converse. An analogous argument applies to $f$ in $G_{x}$, with $K_{b}$ and $H_{a}$ in place of $H_{b}$ and $K_{a}$ respectively, so that

$$
H_{b}=K_{a} \quad \text { and } \quad K_{b}=H_{a} .
$$

Similarly, for each element $f$ in $G_{y}$,

$$
\begin{array}{rll}
f \in X_{b} & \text { if and only if } & f \leq b ; b^{\smile}, \\
\text { if and only if } & f \leq a^{\smile} ; a, \\
\text { if and only if } & f \in Y_{a},
\end{array}
$$

by the definitions of the set $X_{b}$, the element $b$, and the set $Y_{a}$. An analogous argument applies to $f$ in $G_{x}$, with $Y_{b}$ and $X_{a}$ in place of $X_{b}$ and $Y_{a}$ respectively, so that

$$
X_{b}=Y_{a} \quad \text { and } \quad Y_{b}=X_{a} .
$$

Combine (2) with (3), and use the assumed regularity of $a$, together with Corollary 4.3 , to arrive at

$$
H_{b}=K_{a}=Y_{a}=X_{b} \quad \text { and } \quad K_{b}=H_{a}=X_{a}=Y_{b} .
$$

With the help of Corollary 4.3 (with $b$ in place of $a$ ), conclude from (1), (4), and (2) that $b$ is a regular element below $y ; 1 ; x$, with left and right stabilizers as in (2).

To prove the final assertion of the theorem, consider cosets $H_{\xi}$ and $K_{\eta}$ of $H_{a}$ and $K_{a}$ respectively. The equations in (2) imply that $H_{\xi}$ and $K_{\eta}$ are also cosets of $K_{b}$ and $H_{b}$ respectively. Use the definition of $\varphi_{a}$ in Convention 5.5, the involution 
laws, the definition of $b$, the definition of $\varphi_{b}$, and the isomorphism properties of $\varphi_{b}$ to obtain

$$
\begin{array}{rll}
\varphi_{a}\left(H_{\xi}\right)=K_{\eta} \quad \text { if and only if } & H_{\xi} ; a=a ; K_{\eta}, \\
& \text { if and only if } & a^{\smile} ; H_{\xi}^{\smile}=K_{\eta}^{\smile} ; a^{\smile}, \\
& \text { if and only if } & b ; H_{\xi}^{\smile}=K_{\eta}^{\smile} ; b, \\
\text { if and only if } & \varphi_{b}\left(K_{\eta}^{\smile}\right)=H_{\xi}^{\smile}, \\
\text { if and only if } & \varphi_{b}\left(K_{\eta}\right)=H_{\xi} .
\end{array}
$$

The equivalence of the first and last equations implies that the isomorphisms $\varphi_{b}$ and $\varphi_{a}$ are inverses of one another.

Convention 5.12. Continue with the assumption that $a \leq x ; 1 ; y$ is a regular element with normal stabilizers, and write $b=a^{\smile}$. Converse Theorem 5.11 implies that

$$
H_{b}=K_{a} \quad \text { and } \quad K_{b}=H_{a} .
$$

It also implies that if

$$
\left\langle H_{a, \xi}: \xi<\kappa\right\rangle \quad \text { and } \quad\left\langle K_{a, \xi}: \xi<\kappa\right\rangle
$$

are coset systems for $H_{a}$ and $K_{a}$ in $G_{x}$ and $G_{y}$ respectively such that

$$
\varphi_{a}\left(H_{a, \xi}\right)=K_{a, \xi}
$$

for each $\xi$, then by putting

$$
H_{b, \xi}=K_{a, \xi} \quad \text { and } \quad K_{b, \xi}=H_{a, \xi}
$$

for each $\xi$, we arrive at cosets systems

$$
\left\langle H_{b, \xi}: \xi<\kappa\right\rangle \quad \text { and } \quad\left\langle K_{b, \xi}: \xi<\kappa\right\rangle
$$

for $H_{b}$ and $K_{b}$ in $G_{y}$ and $G_{x}$ respectively such that

$$
\varphi_{b}\left(H_{b, \xi}\right)=K_{b, \xi}
$$

for each $\xi$. In what follows, we shall always assume that the coset systems for $H_{b}$ and $K_{b}$ have been chosen so that (1) holds.

The next goal is to prove, for the relative product of two regular elements with normal stabilizers, the analogue of Theorems 5.10 and 5.11. In more detail, suppose that

$$
a \leq x ; 1 ; y \quad \text { and } \quad b \leq y ; 1 ; z
$$

are regular elements with normal stabilizers. The right stabilizer $K_{a}$ of $a$, and the left stabilizer $H_{b}$ of $b$, are normal subgroups $G_{y}$, and therefore so is the product subgroup $K_{a} ; H_{b}$, which includes both $K_{a}$ and $H_{b}$ as subgroups. The quotient isomorphisms $\varphi_{a}^{-1}$ from $G_{y} / K_{a}$ to $G_{x} / H_{a}$, and $\varphi_{b}$ from $G_{y} / H_{b}$ to $G_{z} / K_{b}$ induce isomorphisms $\hat{\varphi}_{a}^{-1}$ and $\hat{\varphi}_{b}$ on the quotient group $G_{y} /\left(K_{a} ; H_{b}\right)$. The goal is to prove that $a ; b$ is a regular element below $x ; 1 ; z$ with normal stabiizers

$$
H_{a ; b}=\hat{\varphi}_{\alpha}^{-1}\left[K_{a} ; H_{b}\right] \quad \text { and } \quad K_{a ; b}=\hat{\varphi}_{b}\left[K_{a} ; H_{b}\right],
$$

and the isomorphism $\varphi_{a ; b}$ from $G_{x} / H_{a ; b}$ to $G_{z} / K_{a ; b}$ coincides with the relational composition $\hat{\varphi}_{a} \mid \hat{\varphi}_{b}$ of the two induced isomorphisms $\hat{\varphi}_{a}$ and $\hat{\varphi}_{b}$.

We begin with a lemma. 
Lemma 5.13. Let $x, y$, and $z$ be measurable atoms. If

$$
a \leq x ; 1 ; y \quad \text { and } \quad b \leq y ; 1 ; z
$$

are regular elements with normal stabilizers, then for all elements $f$ in $G_{x}$ and $h$ in $G_{z}$ the following conditions are equivalent.

(i) $f ; a ; b=a ; b ; h$.

(ii) There are elements $g_{1}$ and $g_{2}$ belonging to the same coset of $K_{a} ; H_{b}$ in $G_{y}$ such that

$$
f ; a=a ; g_{1} \quad \text { and } \quad g_{2} ; b=b ; h .
$$

(iii) There is an element $g$ in $G_{y}$ such that

$$
f ; a=a ; g \quad \text { and } \quad g ; b=b ; h .
$$

Proof. Let $f$ and $h$ be elements of $G_{x}$ and $G_{z}$ respectively. It is easy to establish the implication from (iii) to (i): if

$$
f ; a=a ; g \quad \text { and } \quad g ; b=b ; h,
$$

then

$$
f ; a ; b=a ; g ; b=a ; b ; h .
$$

Assume now that (i) holds, with the goal of establishing (ii). The element $f$ is in $G_{x}$, and therefore belongs to some coset $H_{\xi}$ of $H_{a}$. Apply Isomorphism Theorem 5.4 and Convention 5.5 to write

$$
H_{\xi} ; a=a ; K_{\xi} .
$$

Take $g_{1}$ to be any element in $K_{\xi}$, and observe that the first equation in (ii) holds, by (1) and the remark following Corollary 3.10. An analogous argument produces an element $g_{2}$ in $G_{y}$ such that the second equation in (ii) holds. It remains to verify that $g_{1}$ and $g_{2}$ belong to the same coset of $K_{a} ; H_{b}$. First of all,

$$
\begin{aligned}
a^{\smile} ; f ; a ; b ; b^{\smile}=a^{\smile} ; a ; g_{1} ; b ; b^{\smile} & \\
& =\left(\sum K_{a}\right) ; g_{1} ;\left(\sum H_{b}\right)=\sum K_{a} ; g_{1} ; H_{b},
\end{aligned}
$$

by the choice of $g_{1}$, the assumed regularity of $a$ and $b$, and complete distributivity. An analogous argument shows that

$$
\begin{aligned}
a^{\smile} ; a ; b ; h ; b^{\smile}=a^{\smile} ; a ; g_{2} ; b ; b^{\smile} & \\
& =\left(\sum K_{a}\right) ; g_{2} ;\left(\sum H_{b}\right)=\sum K_{a} ; g_{2} ; H_{b} .
\end{aligned}
$$

The assumption in (i) implies that

$$
a^{\smile} ; f ; a ; b ; b^{\smile}=a^{\smile} ; a ; b ; h ; b^{\smile} .
$$

Combine (2)-(4) to arrive at

$$
\sum K_{a} ; g_{1} ; H_{b}=\sum K_{a} ; g_{2} ; H_{b} .
$$

Sums of sets of atoms can only be equal when the sets themselves are equal, so the preceding equation implies that

$$
K_{a} ; g_{1} ; H_{b}=K_{a} ; g_{2} ; H_{b} .
$$

Use (5) and the assumption that the right stabilizer $K_{a}$ is a normal subgroup to conclude that

$$
g_{1} ; K_{a} ; H_{b}=K_{a} ; g_{1} ; H_{b}=K_{a} ; g_{2} ; H_{b}=g_{2} ; K_{a} ; H_{b} .
$$


The equality of the first and last terms implies that $g_{1}$ and $g_{2}$ belong to the same coset of $K_{a} ; H_{b}$.

Turn, finally, to the implication from (ii) to (iii). If $g_{1}$ and $g_{2}$ are in the same coset of $K_{a} ; H_{b}$, then by interchanging the first two terms, and also the last two terms, in (6), one checks that (5) holds. The element $g_{1}$ belongs to the coset $K_{a} ; g_{1} ; H_{b}$, because the identity element $y$ of $G_{y}$ belongs to both $K_{a}$ and $H_{b}$, and

$$
g_{1}=y ; g_{1} ; y \text {. }
$$

It follows from (5) that $g_{1}$ belongs to $K_{a} ; g_{2} ; H_{b}$, so there must be elements $g_{a}$ in $K_{a}$ and $g_{b}$ in $H_{b}$ such that

$$
g_{1}=g_{a} ; g_{2} ; g_{b} .
$$

Put

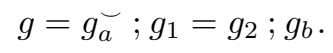

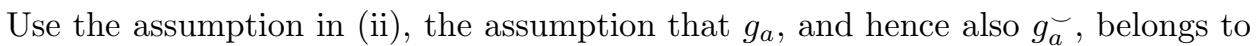
$K_{a}$, the definition of $K_{a}$ as the right stabilizer of $a$, and (7) to obtain

$$
f ; a=a ; g_{1}=a ; g_{a}^{\smile} ; g_{1}=a ; g .
$$

Similarly, use (ii), the assumption that $g_{b}$ is in $H_{b}$, the definition of $H_{b}$ as the left stabilizer of $b$, and (7) to obtain

$$
b ; h=g_{2} ; b=g_{2} ; g_{b} ; b=g ; b
$$

Thus, (iii) holds.

In the statement of the next theorem, $\varphi_{a}$ is assumed to be an isomorphism from $G_{x} / H_{a}$ to $G_{y} / K_{a}$ that induces an isomorphism $\hat{\varphi}_{a}$ from $G_{x} / H_{a ; b}$ to $G_{y} /\left(K_{a} ; H_{b}\right)$, while $\varphi_{b}$ is an isomorphism from $G_{y} / H_{b}$ to $G_{z} / K_{b}$ that induces an isomorphism $\hat{\varphi}_{b}$ from $G_{y} /\left(K_{a} ; H_{b}\right)$ to $G_{z} / K_{a ; b}$.

Theorem 5.14 (First Relative Product Theorem). Let $x, y$, and $z$ be measurable atoms. If

$$
a \leq x ; 1 ; y \quad \text { and } \quad b \leq y ; 1 ; z
$$

are regular elements with normal stabilizers, and in particular if they are atoms, then the relative product $a ; b$ is a regular element below $x ; 1 ; z$ with normal stabilizers

$$
H_{a ; b}=\varphi_{a}^{-1}\left[K_{a} ; H_{b}\right] \quad \text { and } \quad K_{a ; b}=\varphi_{b}\left[K_{a} ; H_{b}\right],
$$

and with quotient isomorphism

$$
\varphi_{a ; b}=\hat{\varphi}_{a} \mid \hat{\varphi}_{b} .
$$

Proof. The stabilizers $H_{a}$ and $K_{b}$ are assumed to be normal subgroups of $G_{y}$, so the product group $K_{a} ; H_{b}$ is also a normal subgroup of $G_{y}$. It can of course be written as the union of distinct cosets $K_{\xi}$ of $K_{a}$, say with $\xi<\kappa$, so that

$$
K_{a} ; H_{b}=\bigcup_{\xi<\kappa} K_{\xi} .
$$

Write

$$
H_{\xi}=\varphi_{a}^{-1}\left(K_{\xi}\right)
$$

for $\xi<\kappa$, and use the definition of $\varphi_{a}$ and Convention 5.5 to obtain

$$
H_{\xi} ; a=a ; K_{\xi}
$$


for each $\xi$. The first goal is to prove that $a ; b$ is left-regular, by showing that

$$
X_{a ; b}=\bigcup_{\xi<\kappa} H_{\xi}=H_{a ; b} .
$$

Since $K_{\xi}$ is a coset of $K_{a}$ in $K_{a} ; H_{b}$, there must be an element $h_{\xi}$ in $H_{b}$ such that

$$
K_{\xi}=K_{a} ; h_{\xi}
$$

Use (3), (5), the definition of $K_{a}$ as the right stabilizer of $a$, and the assumption that $h_{\xi}$ belongs to the left stabilizer of $b$, to get

$$
H_{\xi} ; a ; b=a ; K_{\xi} ; b=a ; K_{a} ; h_{\xi} ; b=a ; h_{\xi} ; b=a ; b
$$

This argument shows that $H_{\xi}$ is included in the left stabilizer $H_{a ; b}$ for every $\xi<\kappa$, and therefore

$$
\bigcup_{\xi<\kappa} H_{\xi} \subseteq H_{a ; b}
$$

Compute:

$$
\begin{aligned}
\sum X_{a ; b} & =(a ; b) ;(a ; b)^{\smile}=a ; b ; b^{\smile} ; a^{\smile} \\
& =a ;\left(\sum H_{b}\right) ; a^{\smile}=a ; K_{a} ;\left(\sum H_{b}\right) ; a^{\smile} \\
& =\sum a ; K_{a} ; H_{b} ; a^{\smile}=\sum a ;\left(\bigcup_{\xi<\kappa} K_{\xi}\right) ; a^{\smile} \\
& =\sum_{\xi<\kappa} a ; K_{\xi} ; a^{\smile}=\sum_{\xi<\kappa} H_{\xi} ; a ; a^{\smile} \\
& =\sum_{\xi<\kappa} H_{\xi} ;\left(\sum H_{a}\right)=\sum\left(\bigcup_{\xi<\kappa}\left(H_{\xi} ; H_{a}\right)\right)=\sum\left(\bigcup_{\xi<\kappa} H_{\xi}\right),
\end{aligned}
$$

by the definition of $X_{a ; b}$, the second involution law and the associative law, the left-regularity of $b$, the definition of $K_{a}$ as the right stabilizer of $a$, complete distributivity, (1), complete distributivity, (3), the left-regularity of $a$, complete distributivity, and the fact that $H_{a}$ is the identity coset of the quotient $G_{x} / H_{a}$. Since $X_{a ; b}$ and $\bigcup_{\xi<\kappa} H_{\xi}$ are both sets of atoms, it follows from (7) that these sets must be equal. Consequently,

$$
\bigcup_{\xi<\kappa} H_{\xi} \subseteq H_{a ; b} \subseteq X_{a ; b}=\bigcup_{\xi<\kappa} H_{\xi}
$$

by (6), Lemma 4.5(ii), and the remark following (7). The first and last terms are the same, so equality holds everywhere, which proves (4).

Use (4) and Corollary 4.3 to conclude that the relative product $a ; b$ is left-regular. Also,

$$
H_{a ; b}=\bigcup_{\xi<\kappa} H_{\xi}=\bigcup_{\xi<\kappa} \varphi_{a}^{-1}\left(K_{\xi}\right)=\varphi_{a}^{-1}\left[\bigcup_{\xi<\kappa} K_{\xi}\right]=\varphi_{a}^{-1}\left[K_{a} ; H_{b}\right],
$$

by (4), (2), the preservation of unions under inverse images, and (1).

The group $K_{a} ; H_{b}$ is normal in $G_{y}$, so the quotient $\left(K_{a} ; H_{b}\right) / K_{a}$ is a normal subgroup of $G_{y} / K_{a}$. Because $\varphi_{a}$ maps $G_{x} / H_{a}$ isomorphically to $G_{y} / K_{a}$, the inverse image of $\left(K_{a} ; H_{b}\right) / K_{a}$ under $\varphi_{a}$, which is $H_{a ; b} / H_{a}$, by (8) and the definition of $\varphi_{a}$, must be a normal subgroup of $G_{x} / H_{a}$, and therefore $H_{a ; b}$ must be a normal subgroup of $G_{x}$, by group theory.

It has been shown that the relative product $a ; b$ is left-regular and that its left stabilizer $H_{a ; b}$ is normal in $G_{x}$ and coincides with $\varphi_{a}^{-1}\left[K_{a} ; H_{b}\right]$. A symmetric argument shows that $a ; b$ is right-regular, and that its right stabilizer $K_{a ; b}$ is normal in $G_{z}$ and coincides with $\varphi_{b}\left[K_{a} ; H_{b}\right]$. It remains to prove that

$$
\varphi_{a ; b}=\hat{\varphi}_{a} \mid \hat{\varphi}_{b}
$$


Write $c=a ; b ; b^{-}$, and observe that $c \leq x ; 1 ; y$, since

$$
\begin{aligned}
c=a ; b ; b^{-} \leq(x ; 1 ; y) ;(y ; 1 ; z) & ;(y ; 1 ; z)^{\smile} \\
& =(x ; 1 ; y) ;(y ; 1 ; z) ;(z ; 1 ; y) \leq x ; 1 ; y,
\end{aligned}
$$

by the definition of $c$, the assumptions on $a$ and $b$, monotony, and Lemma 2.5(iii),(iv). Also,

$$
c=a ; b ; b^{-}=a ;\left(\sum H_{b}\right)=\sum a ; H_{b},
$$

by the left-regularity of $b$ and complete distributivity. Compute:

$$
\begin{aligned}
\sum X_{c}=c ; c^{\smile}=\left(a ; b ; b^{\smile}\right) ;\left(a ; b ; b^{\smile}\right)^{\smile} & \\
=a & ; b ; b^{\smile} ; b ; b^{\smile} ; a^{\smile}=a ; b ;\left(\sum K_{b}\right) ; b^{\smile} ; a^{\smile} \\
& =\sum a ; b ; K_{b} ; b^{\smile} ; a^{\smile}=a ; b ; b^{\smile} ; a^{\smile} \\
& =(a ; b) ;(a ; b)^{\smile}=\sum H_{a ; b}
\end{aligned}
$$

by the definition of $X_{c},(10)$, the two involution laws and the associative law, the right-regularity of $b$, complete distributivity, the definition of $K_{b}$ as the right stabilizer of $b$, the second involution law and the associative law, and the leftregularity of $a ; b$. Because $X_{c}$ and $H_{a ; b}$ are sets of atoms, it follows from (11) that $X_{c}=H_{a ; b}$. Use this observation, the definition of $c$ and Lemma 3.11 (with $a ; b$ and $b^{\smile}$ in place of $a$ and $b$ respectively), and Lemma 4.5(ii) (with $c$ in place of $a$ ) to arrive at

$$
X_{c}=H_{a ; b} \subseteq H_{c} \subseteq X_{c} .
$$

The first and last sets are equal, so equality holds everywhere. Apply Corollary 4.3 to conclude that $c$ is a left-regular element with normal left stabilizer $H_{c}=H_{a ; b}$.

The proof that $c$ is right-regular with normal right stabilizer $K_{c}=K_{a} ; H_{b}$ involves a similar computation. In more detail,

$$
\begin{aligned}
& \sum Y_{c}=c^{\smile} ; c=\left(a ; b ; b^{\smile}\right)^{-} ;\left(a ; b ; b^{\smile}\right) \\
&=b ; b^{\smile} ; a^{\smile} ; a ; b ; b^{-}=\left(\sum H_{b}\right) ;\left(\sum K_{a}\right) ;\left(\sum H_{b}\right) \\
& \quad=\sum H_{b} ; K_{a} ; H_{b}=\sum K_{a} ; H_{b} ; H_{b}=\sum K_{a} ; H_{b},
\end{aligned}
$$

by the definition of $Y_{c}$, the definition of $c$, the two involution laws and the associative law, the right-regularity of $a$ and the left-regularity of $b$, complete distributivity, the assumption that $K_{a}$ is a normal subgroup of $G_{y}$, and Corollary 3.10 (with $b$ in place of $a$ ), which implies that $H_{b}$ is a subgroup of $G_{y}$ and hence closed under the group operaton of relative multiplication. The sets $Y_{c}$ and $K_{a} ; H_{b}$ consist of atoms in $G_{y}$, so (12) implies that these sets are equal,

$$
Y_{c}=K_{a} ; H_{b} .
$$

The next step is to check that

$$
K_{a} ; H_{b} \subseteq K_{c} .
$$

Consider an element $g$ in $K_{a} ; H_{b}$, and let $k$ in $K_{a}$ and $h$ in $H_{b}$ be elements such that

$$
g=k ; h .
$$


Compute:

$$
\begin{aligned}
c ; g=\sum a ; H_{b} ; g=\sum a ; H_{b} & ; k ; h \\
& =\sum a ; k ; H_{b} ; h=\sum a ; H_{b} ; h=\sum a ; H_{b}=c
\end{aligned}
$$

by (10) and complete distributivity, (15), the assumption that $H_{b}$ is normal in $G_{y}$, the assumption that $k$ is in the right stabilizer $K_{a}$ of $a$, the assumption that $h$ is in the subgroup $H_{b}$ of $b$, which is closed under the group operation of relative multiplication, and (10). It follows from this computation that $g$ belongs to the right stabilizer $K_{c}$, which completes the proof of (14).

Use (13), (14), and the right-regular version of Lemma 4.5(ii) to arrive at

$$
Y_{c}=K_{a} ; H_{b} \subseteq K_{c} \subseteq Y_{c} .
$$

The first and last sets are equal, so equality holds everywhere. Apply Corollary 4.3 (with $c$ in place of $a$ ) to conclude that $c$ is a right-regular element with normal right stabilizer $K_{c}=K_{a} ; H_{b}$.

The element $a \leq x ; 1 ; y$ is regular with normal stabilizers, by assumption, and the same properties have been established for the element $c$. Moreover,

$$
a=a ; y \leq a ; b ; b^{\smile}=c
$$

by Lemma 2.3(iii),(i) (with $b, y$, and $z$ in place of $a, x$, and $y$ respectively), and the definition of $c$. Apply Refinement Theorem 5.9 (with $c$ in place of $b$ ) to conclude that the isomorphism $\hat{\varphi}_{a}$ from $G_{x} / H_{c}$ to $G_{y} / K_{c}$ induced by $\varphi_{a}$ coincides with $\varphi_{c}$, in symbols,

$$
\varphi_{c}=\hat{\varphi}_{a} .
$$

Now write $d=a^{\smile} ; a ; b$. In an entirely analogous fashion, one shows that $d$ is a regular element below $y ; 1 ; z$ with normal left and right stabilizers

$$
H_{d}=K_{a} ; H_{b} \quad \text { and } \quad K_{d}=K_{a ; b},
$$

and therefore, since $b \leq d$,

$$
\varphi_{d}=\hat{\varphi}_{b}
$$

by Refinement Theorem 5.9.

The last step in the link is to prove that

$$
\varphi_{a ; b}=\varphi_{c} \mid \varphi_{d}
$$

Recall that $\varphi_{a ; b}$ maps $G_{x} / H_{a ; b}$ isomorphically to $G_{z} / K_{a ; b}$. Fix an element $f$ in $G_{x}$, and select an element $h$ in $G_{z}$ so that the coset $f ; H_{a ; b}$ of the normal subgroup $H_{a ; b}$ is mapped by $\varphi_{a ; b}$ to the coset $K_{a ; b} ; h$ of the normal subgroup $K_{a ; b}$. It follows from the definition of $\varphi_{a ; b}$ and Convention 5.5 (with $a ; b$ in place of $a$ ) that

$$
f ; H_{a ; b} ; a ; b=a ; b ; K_{a ; b} ; h .
$$

Use the left- and right-regular versions of Corollary 3.10 and the remark following it, together with (19), to arrive at

$$
f ; a ; b=\left(f ; H_{a ; b}\right) ; a ; b=a ; b ;\left(K_{a ; b} ; h\right)=a ; b ; h .
$$

Use (20) and the implication from (i) to (iii) in Lemma 5.13 to obtain an element $g$ in $G_{y}$ such that

$$
f ; a=a ; g \quad \text { and } \quad g ; b=b ; h .
$$


Compute:

$$
\begin{aligned}
f ; c=f ; a ;\left(\sum H_{b}\right)=a ; g ;\left(\sum H_{b}\right) \\
=\sum a ; g ; H_{b}=\sum a ; H_{b} ; g=a ;\left(\sum H_{b}\right) ; g=c ; g
\end{aligned}
$$

by $(10),(21)$, complete distributivity, the assumption that $H_{b}$ is normal, complete distributivity, and (10). Use the left- and right-regular versions of Corollary 3.10 and the remark following it, together with $(22)$, to arrive at

$$
\left(f ; H_{c}\right) ; c=f ; c=c ; g=c ;\left(K_{c} ; g\right) \text {. }
$$

Apply the definition of $\varphi_{c}$ and Convention 5.5 (with $c$ in place of $a$ ) to conclude from (23) that $\varphi_{c}$ maps the coset $f ; H_{c}$ of $H_{c}$ to the coset $K_{c} ; g$ of $K_{c}$. Use the equations

$$
H_{c}=H_{a ; b} \quad \text { and } \quad K_{c}=K_{a} ; H_{b}
$$

established earlier in the proof to conclude that

$$
\varphi_{c}\left(f ; H_{a ; b}\right)=\varphi_{c}\left(f ; H_{c}\right)=K_{c} ; g=K_{a} ; H_{b} ; g .
$$

In an entirely analogous fashion, one shows that

$$
\varphi_{d}\left(g ; K_{a} ; H_{b}\right)=K_{a ; b} ; h .
$$

The subgroup $K_{b} ; H_{b}$ is normal in $G_{y}$, so the cosets $g ; K_{a} ; H_{b}$ and $K_{a} ; H_{b} ; g$ are equal. Combine this observation with the preceding equations to see that the composite isomorphism $\varphi_{c} \mid \varphi_{d}$ maps the coset $f ; H_{a ; b}$ to the coset $K_{a ; b} ; h$, just as does the isomorphism $\varphi_{a ; b}$, by (19) and Convention 5.5 (with $a ; b$ in place of $a$ ). Consequently, the two isomorphisms coincide. This proves (18).

Together, (16)-(18) immediately yield (9):

$$
\varphi_{a ; b}=\varphi_{c}\left|\varphi_{d}=\hat{\varphi}_{a}\right| \hat{\varphi}_{b} .
$$

This completes the proof of the theorem.

Relative Product Theorem 5.14 can be combined with Refinement Theorem 5.9 and Isomorphism Theorem 5.6 to obtain an important characterization of the relational composition of two quotient isomorphisms. Recall the notation introduced before Theorem 5.6: the inner automorphism of a quotient group $G_{x} / H_{c}$ determined by a coset $H_{\eta}$ of $H_{c}$ is the mapping $\tau$ defined by

$$
\tau\left(H_{\xi}\right)=H_{\eta}^{\smile} ; H_{\xi} ; H_{\eta}
$$

for all cosets $H_{\xi}$ of $H_{c}$. This inner automorphism induces an inner automorphism $\hat{\tau}$ of the quotient group $G_{x} / H_{a ; b}$ under the assumption that $H_{c}$ is a subgroup of $H_{a ; b}$. In the statement of the next theorem, the mappings $\hat{\varphi}_{a}$ and $\hat{\varphi}_{b}$ are the isomorphism described before Relative Product Theorem 5.14, while $\hat{\varphi}_{c}$ is the isomorphism from $G_{x} / H_{a ; b}$ to $G_{z} / K_{a ; b}$ induced by the isomorphism $\varphi_{c}$ from $G_{x} / H_{c}$ to $G_{z} / K_{c}$, and $\hat{\tau}$ is the inner automorphism of $G_{x} / H_{a ; b}$ induced by an inner automorphism $\tau$ of $G_{x} / H_{c}$.

Theorem 5.15 (Second Relative Product Theorem). Let $x, y$, and $z$ be measurable atoms, and

$$
a \leq x ; 1 ; y, \quad b \leq y ; 1 ; z, \quad c \leq x ; 1 ; z
$$

regular elements with normal stabilizers. In particular, they may be atoms. If

$$
H_{\eta} ; c \leq a ; b
$$


for some coset $H_{\eta}$ of $H_{c}$, then

$$
H_{a} ; H_{c} \subseteq \varphi_{a}^{-1}\left[K_{a} ; H_{b}\right] \quad \text { and } \quad K_{b} ; K_{c} \subseteq \varphi_{b}\left[K_{a} ; H_{b}\right],
$$

and

$$
\hat{\varphi}_{a}\left|\hat{\varphi}_{b}=\hat{\tau}\right| \hat{\varphi}_{c}
$$

where $\tau$ is the inner automorphism of $G_{x} / H_{c}$ determined by $H_{\eta}$.

Proof. The assumption that $a$ and $b$ are regular elements with normal stabilizers implies that $a ; b$ is a regular element with normal stabilizers

$$
H_{a ; b}=\varphi_{a}^{-1}\left[K_{a} ; H_{b}\right] \quad \text { and } \quad K_{a ; b}=\varphi_{b}\left[K_{a} ; H_{b}\right],
$$

and that

$$
\varphi_{a ; b}=\hat{\varphi}_{a} \mid \hat{\varphi}_{b},
$$

by Relative Product Theorem 5.14.

The element $c$ is assumed to be regular with normal stabilizers, so its left translation

$$
d=H_{\eta} ; c \leq a ; b,
$$

is also regular with normal stabilizers, and in fact,

$$
H_{d}=H_{c} \quad \text { and } \quad K_{d}=K_{c},
$$

by Translation Lemma 4.15(i). These observations show that Refinement Theorem 5.9 may be applied to (3) to conclude, first, that

$$
H_{d} \subseteq H_{a ; b} \quad \text { and } \quad K_{d} \subseteq K_{a ; b},
$$

and, second, that the isomorphism $\varphi_{d}$ from $G_{x} / H_{d}$ to $G_{z} / K_{d}$ induces an isomorphism $\hat{\varphi}_{d}$ from $G_{x} / H_{a ; b}$ to $G_{z} / K_{a ; b}$ with the property

$$
\hat{\varphi}_{d}=\varphi_{a ; b} .
$$

Combine (2) and (6) to arrive at

$$
\hat{\varphi}_{d}=\hat{\varphi}_{a} \mid \hat{\varphi}_{b} .
$$

Isomorphism Theorem 5.6 (with $z, c$, and $d$ in place of $y, a$, and $b$ respectively) says that

$$
\varphi_{d}=\tau \mid \varphi_{c}
$$

where $\tau$ is the inner automorphism of $G_{x} / H_{c}$ determined by the coset $H_{\eta}$. The inclusions in (4) and (5) imply that

$$
H_{c} \subseteq H_{a ; b} \quad \text { and } \quad K_{c} \subseteq K_{a ; b},
$$

so the inner automorphism $\tau$ of $G_{x} / H_{c}$ induces an inner automorphism $\hat{\tau}$ of $G_{x} / H_{a ; b}$, and the isomorphism $\varphi_{c}$ from $G_{x} / H_{c}$ to $G_{z} / K_{c}$ induces an isomorphism $\hat{\varphi}_{c}$ from $G_{x} / H_{a ; b}$ to $G_{z} / K_{a ; b}$, and

$$
\hat{\varphi}_{d}=\hat{\tau} \mid \hat{\varphi}_{c}
$$

by (8). Combine (7) and (10) to arrive at

$$
\hat{\varphi}_{a}\left|\hat{\varphi}_{b}=\hat{\tau}\right| \hat{\varphi}_{c} .
$$


Together, (1), (4), and (5) show that $H_{c}$ and $K_{c}$ are subgroups of

$$
\varphi_{a}^{-1}\left[K_{a} ; H_{b}\right] \quad \text { and } \quad \varphi_{b}\left[K_{a} ; H_{b}\right]
$$

respectively. Since $K_{a}$ and $H_{b}$ are subgroups of $K_{a} ; H_{b}$, the two groups in (11) also include $\varphi_{a}^{-1}\left[K_{a}\right]$ and $\varphi_{b}\left[H_{b}\right]$ respectively, that is to say, they include $H_{a}$ and $K_{b}$, by the definitions of $\varphi_{a}$ and $\varphi_{b}$. Use these observation to arrive at

$$
H_{a} ; H_{c} \subseteq \varphi_{a}^{-1}\left[K_{a} ; H_{b}\right] \quad \text { and } \quad K_{b} ; K_{c} \subseteq \varphi_{b}\left[K_{a} ; H_{b}\right] .
$$

The special case of the theorem in which $c$ is below $a ; b$ is important enough to merit separate formulation. In this case, the translating coset $H_{\eta}$ is the identity coset $H_{c}$, and therefore the inner automorphism $\tau$ determined by this coset is the identity automorphism of $G_{x} / H_{c}$.

Corollary 5.16. Let $x, y$, and $z$ be measurable atoms, and

$$
a \leq x ; 1 ; y, \quad b \leq y ; 1 ; z, \quad c \leq x ; 1 ; z
$$

regular elements with normal stabilizers. If $c \leq a ; b$, then $\hat{\varphi}_{a} \mid \hat{\varphi}_{b}=\hat{\varphi}_{c}$.

Another special case of Relative Product Theorem 5.15 is when one of the quotient groups

$$
G_{x} / H_{a ; b}, \quad G_{y} /\left(K_{a} ; H_{b}\right), \quad G_{z} / K_{a ; b}
$$

is abelian (and hence all of them are abelian, since they are isomorphic to one another). In this case, the inner automorphism $\hat{\tau}$ mentioned in the theorem is again the identity automorphism, and therefore may be omitted from the final equation of the theorem.

Corollary 5.17. Let $x, y$, and $z$ be measurable atoms, and

$$
a \leq x ; 1 ; y, \quad b \leq y ; 1 ; z, \quad \text { and } \quad c \leq x ; 1 ; z
$$

regular elements with normal stabilizers. If the quotient group $G_{x} / H_{a ; b}$ is abelian, then

$$
\hat{\varphi}_{a} \mid \hat{\varphi}_{b}=\hat{\varphi}_{c}
$$

whenever some translation of $c$ is below $a ; b$.

Relative Product Theorem 5.14 says that, under suitable hypotheses, the inverse image of $K_{a} ; H_{b}$ under $\varphi_{a}^{-1}$ is $H_{a ; b}$, and the image of $K_{a} ; H_{b}$ under $\varphi_{b}$ is $K_{a ; b}$. The following corollary gives, for atoms, an alternative description of these images.

Theorem 5.18 (Image Theorem). Let $x, y$, and $z$ be measurable atoms. If

$$
a \leq x ; 1 ; y, \quad b \leq y ; 1 ; z, \quad c \leq x ; 1 ; z
$$

are all atoms, then

$$
\varphi_{a}\left[H_{a} ; H_{c}\right]=K_{a} ; H_{b}, \quad \varphi_{b}\left[K_{a} ; H_{b}\right]=K_{b} ; K_{c},
$$

and

In particular,

$$
\varphi_{c}\left[H_{a} ; H_{c}\right]=K_{b} ; K_{c}
$$

$$
H_{a ; b}=H_{a} ; H_{c} \quad \text { and } \quad K_{a ; b}=K_{b} ; K_{c} .
$$


Proof. Consider elements $a, b$, and $c$ satisfying the hypotheses of the theorem. All three are regular elements with normal stabilizers, by Corollaries 4.7 and 4.16 . The relative product $a ; b$ is a regular element below $x ; 1 ; z$ with normal stabilizers, by Relative Product Theorem 5.14, and in particular, it is non-zero, by Lemma 4.4. The left-translations of $c$ are atoms that partition $x ; 1 ; z$, by Atomic Partition Lemma 4.11, so one of these left translations must be below the non-zero element $a ; b$. Apply Relative Product Theorem 5.15 to obtain

$$
H_{a} ; H_{c} \subseteq \varphi_{a}^{-1}\left[K_{a} ; H_{b}\right] \quad \text { and } \quad K_{b} ; K_{c} \subseteq \varphi_{b}\left[K_{a} ; H_{b}\right] .
$$

The converses $a^{\smile}, b^{\smile}$, and $c^{\smile}$ are atoms below

$$
y ; 1 ; x, \quad z ; 1 ; y, \quad \text { and } \quad z ; 1 ; x
$$

respectively, by Lemmas 2.1(vi) and 2.5(iii), the assumptions on $a, b$, and $c$, and monotony. Apply the argument from the preceding paragraph to the atoms $a^{-}, c$, and $b$ in place of $a, b$, and $c$ respectively to get

$$
H_{a \smile} ; H_{b} \subseteq \varphi_{a}^{-1}\left[K_{a \smile} ; H_{c}\right] \quad \text { and } \quad K_{c} ; K_{b} \subseteq \varphi_{c}\left[K_{a \smile} ; H_{c}\right] .
$$

Apply the same argument again to the atoms $b, c^{-}$, and $a^{-}$in place of $a, b$, and $c$ respectively to get

$$
H_{b} ; H_{a} \smile \subseteq \varphi_{b}^{-1}\left[K_{b} ; H_{c} \smile\right] \quad \text { and } \quad K_{c \smile} ; K_{a} \smile \subseteq \varphi_{c}-\left[K_{b} ; H_{c} \smile\right] .
$$

The equations

$$
\begin{aligned}
& \varphi_{a \smile}=\varphi_{a}^{-1}, \quad H_{a \smile}=K_{a}, \quad K_{a} \smile=H_{a}, \\
& \varphi_{c} \smile=\varphi_{c}^{-1}, \quad H_{c} \smile=K_{c}, \quad K_{c} \smile=H_{c}
\end{aligned}
$$

are all valid, by Converse Theorem 5.11, so the inclusions in (2) and (3) may be rewritten in the forms

$$
K_{a} ; H_{b} \subseteq \varphi_{a}\left[H_{a} ; H_{c}\right] \quad \text { and } \quad K_{c} ; K_{b} \subseteq \varphi_{c}\left[H_{a} ; H_{c}\right]
$$

and

$$
H_{b} ; K_{a} \subseteq \varphi_{b}^{-1}\left[K_{b} ; K_{c}\right] \quad \text { and } \quad H_{c} ; H_{a} \subseteq \varphi_{c}^{-1}\left[K_{b} ; K_{c}\right] .
$$

For example, to obtain the first inclusion in (6), use the second equation in (4), the first inclusion in (2), the first equation in (4), and the fact that the inverse of the inverse of $\varphi_{a}$ is $\varphi_{a}$, to arrive at

$$
K_{a} ; H_{b}=H_{a \smile} ; H_{b} \subseteq \varphi_{a}^{-1}\left[K_{a \smile} ; H_{c}\right]=\left(\varphi_{a}^{-1}\right)^{-1}\left[H_{a} ; H_{c}\right]=\varphi_{a}\left[H_{a} ; H_{c}\right] .
$$

The first equations in (1) and (6) yield

$$
\varphi_{a}\left[H_{a} ; H_{c}\right]=K_{a} ; H_{b} .
$$

Similarly, the second equation in (1) and the first equation in (7), together with the assumption that the stabilizers are normal subgroups, yield

$$
\varphi_{b}\left[K_{a} ; H_{b}\right]=K_{b} ; K_{c} .
$$

Finally, the second equations in (6) and (7), and the assumption that the stabilizers are normal, yield

$$
\varphi_{c}\left[H_{a} ; H_{c}\right]=K_{b} ; K_{c}
$$


To obtain the first equation in the final assertion of the theorem, use Relative Product Theorem 5.14 and (8),

$$
H_{a ; b}=\varphi_{a}^{-1}\left[K_{a} ; H_{b}\right]=H_{a} ; H_{c} .
$$

The second equation is obtained in a similar fashion, using (9) instead of (8).

\section{SEMI-SCAFFOLDS AND SCAFFOLDS}

The previous sections take a "local" perspective, and study properties of certain types of elements that lie below rectangles with measurable atoms for sides. The most important elements of this kind are regular elements with normal stabilizers, and in particular, atoms. This and the next sections take a "global" perspective, and focus on the entire measurable relation algebra.

Definition 6.1. A relation algebra is called measurable if the identity element is the sum of measurable atoms. If the identity element is the sum of finitely measurable atoms, then the algebra is said to be finitely measurable.

Fix a complete and atomic, measurable relation algebra $\mathfrak{A}$ for the remainder of the discussion. The assumption of completeness is only needed in order not to have to worry about the existence of certain infinite sums. All elements and operations are assumed to be those of $\mathfrak{A}$. Let $I$ be the set of measurable atoms in $\mathfrak{A}$. The assumption of measurability is expressed symbolically by the equation $1^{\prime}=\sum I$. Define a binary relation $\mathcal{E}$ on the set $I$ by putting

$$
\mathcal{E}=\{(x, y): x, y \in I \text { and } x ; 1 ; y \neq 0\} .
$$

For atoms $x$ and $y$, the conditions

$$
x ; 1 ; y \neq 0 \quad \text { and } \quad 1 ; x ; 1=1 ; y ; 1
$$

are equivalent, by Lemma 2.2(viii), so an alternative definition of $\mathcal{E}$ is given by

$$
\mathcal{E}=\{(x, y): x, y \in I \text { and } 1 ; x ; 1=1 ; y ; 1\} .
$$

This second way of defining $\mathcal{E}$ makes clear that $\mathcal{E}$ is an equivalence relation on the set $I$.

The principal notion that will be in this section is that of a semi-scaffold.

Definition 6.2. A system $a=\left\langle a_{x y}:(x, y) \in \mathcal{E}\right\rangle$ of atoms is called a semi-scaffold if, for all pairs $(x, y)$ in $\mathcal{E}$, and for all atoms $x$ in $I$, the following conditions are satisfied.

(i) $a_{x y} \leq x ; 1 ; y$.

(ii) $a_{x x}=x$.

(iii) $a_{y x}=a_{x y}$.

A semi-scaffold is called a scaffold if, for all pairs $(x, y)$ and $(y, z)$ in $\mathcal{E}$,

(iv) $a_{x z} \leq a_{x y} ; a_{y z}$.

The conditions defining a semi-scaffold and a scaffold can be weakened somewhat. To this end, it is helpful to assume that the set $I$ of measurable atoms is linearly ordered, say by a relation $<$. 
Lemma 6.3. A system $a=\left\langle a_{x y}:(x, y) \in \mathcal{E}\right\rangle$ of atoms is a semi-scaffold if and only if the following assumptions are satisfied.

(i) $a_{x y} \leq x ; 1 ; y$ for all $(x, y)$ in $\mathcal{E}$ with $x<y$.

((ii) $a_{x x}=x$ for all $x$ in $I$.

(iii) $a_{y x}=a_{x y}^{\smile}$ for all $(x, y)$ in $\mathcal{E}$ with $x<y$.

A semi-scaffold is a scaffold if and only if

(iv) $a_{x z} \leq a_{x y} ; a_{y z}$ for all $(x, y)$ and $(y, z)$ in $\mathcal{E}$ with $x<y<z$.

Proof. It is obvious that a system of atoms satisfying conditions (i)-(iii) in the definition of a semi-scaffold satisfies assumptions (i)-(iii) of the lemma. To establish the reverse implication, assume that $a$ is a system of atoms satisfying assumptions (i)-(iii) of the lemma. Fix a pair $(x, y)$ in $\mathcal{E}$. The first step is to verify that semiscaffold condition (i) holds for this pair. If $x<y$, then condition (i) holds by assumption. If $x=y$, then

$$
a_{x y}=a_{x x}=x \leq x ; 1 ; x=x ; 1 ; y,
$$

by the hypothesis of this case, assumption (ii) of the lemma, and Lemma 2.5(i) (with $y=x$ ). If $x>y$, then $a_{y x} \leq y ; 1 ; x$, by assumption (i) of the lemma (with $x$ and $y$ interchanged), and consequently

$$
a_{x y}=a_{y x}^{\smile} \leq(y ; 1 ; x)^{\smile}=x ; 1 ; y,
$$

by assumption (iii) of the lemma (with $x$ and $y$ interchanged), the preceding observation, monotony, and Lemma 2.5(iii). This completes the verification of semiscaffold condition (i).

Semi-scaffold condition (ii) coincides with assumption (ii) of the lemma, so there is nothing to verify. Turn now to the verification of semi-scaffold condition (iii). If $x<y$, then condition (iii) holds by assumption (iii) of the lemma. If $x=y$, then

$$
a_{y x}=a_{x x}=x=x^{\smile}=a_{x x}^{\smile}=a_{x y}^{\smile},
$$

by the hypothesis $x=y$, assumption (ii) of the lemma, and Lemma 2.1(vii). If $x>$ $y$, then $a_{x y}=a_{y x}$, by assumption (iii) of the lemma (with $x$ and $y$ interchanged), and therefore

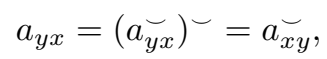

by the first involution law and the preceding observation.

To prove the second assertion of the theorem, assume that the system of atoms $a$ satisfies assumptions (i)-(iii) of the lemma. It then satisfies semi-scaffold conditions (i)-(iii), by the arguments of the previous paragraphs, so it may be assumed that these conditions hold for $a$. The goal is to derive condition (iv) from these conditions and assumption (iv) of the lemma. Consider pairs $(x, y)$ and $(y, z)$ in $\mathcal{E}$. The atom $a_{x z}$ is below $x ; 1 ; z$, by semi-scaffold condition (i), so its domain is $x$, and $x ; a_{x z}=a_{x z}$, by Lemma 2.3. Consequently, if $x=y$, then

$$
a_{x y} ; a_{y z}=a_{x x} ; a_{x z}=x ; a_{x z}=a_{x z},
$$

by the hypothesis $x=y$, semi-scaffold condition (ii), and the preceding observation. A similar argument applies if $y=z$. If $x=z$, then

$$
a_{x y} ; a_{y z}=a_{x y} ; a_{y x}=a_{x y} ; a_{x y}^{\smile}=\sum H_{a_{x y}} \geq x=a_{x x}=a_{x z},
$$

by the hypothesis $x=z$, semi-scaffold condition (iii), the assumption that $a_{x y}$ is an atom and hence regular, by Corollary 4.7 , the fact that the stabilizer $H_{a_{x y}}$ is 
a subgroup of $G_{x}$ and therefore contains the group identity element $x$, and semiscaffold condition (ii). Assume now that $x, y$, and $z$ are distinct. If $x<y<z$, then condition (iv) holds by assumption (iv) of the lemma. If $x<z<y$, then

$$
a_{x y} \leq a_{x z} ; a_{z y}=a_{x z} ; a_{y z}^{\smile},
$$

by the hypothesis of this case, assumption (iv) of the lemma (with $y$ and $z$ interchanged), and semi-scaffold condition (iii). Apply the cycle laws for atoms in Lemma 2.2(v) to arrive at $a_{x z} \leq a_{x y} ; a_{y z}$. Similarly, if say $z<x<y$, then

$$
a_{z y} \leq a_{z x} ; a_{x y}=a_{x z}^{\smile} ; a_{x y},
$$

by the hypothesis of this case, assumption (iv) of the lemma (with $z, x$, and $y$ in place of $x, y$, and $z$ respectively) and semi-scaffold condition (iii) (with $z$ in place of $y$ ). Form the converse of both sides of this inequality, and use semi-scaffold condition (iii) (with $z$ in place of $x$ ), monotony, and the two involution laws to obtain

$$
a_{y z}=a_{z y}^{\smile} \leq\left(a_{x z}^{\smile} ; a_{x y}\right)^{\smile}=a_{x y}^{\smile} ;\left(a_{x z}^{\smile}\right)^{\smile}=a_{x y}^{\smile} ; a_{x z} .
$$

Apply the cycle laws for atoms in Lemma 2.2(v) to arrive at $a_{x z} \leq a_{x y} ; a_{y z}$. The remaining three cases of condition (iv) are treated in a similar fashion.

With the help of the preceding lemma, it is easy to show that every atomic, measurable relation algebra has a semi-scaffold.

Lemma 6.4 (Semi-scaffold Existence Lemma). Every atomic, measurable relation algebra has a semi-scaffold.

Proof. Assume that the set $I$ of measurable atoms is linearly ordered, say by a relation $<$. For each $x$ in $I$, put

$$
a_{x x}=x,
$$

and observe that assumption (ii) of Lemma 6.3 holds. For each pair $(x, y)$ in $\mathcal{E}$ with $x<y$, choose an atom $b \leq x ; 1 ; y$, and put

$$
a_{x y}=b \quad \text { and } \quad a_{y x}=b^{\smile} .
$$

Such an atom $b$ exists because the relation algebra is assumed to be atomic, and the definition of $\mathcal{E}$, together with the assumption that $(x, y)$ is in $\mathcal{E}$, imply that the rectangle $x ; 1 ; y$ is non-zero. Observe that $a_{y x}$ is also an atom, by Lemma 2.1(vi). The choice of $a_{x y}$ and the definition of $a_{y x}$ imply that assumptions (i) and (iii) of Lemma 6.3 hold. Consequently, the system

$$
a=\left\langle a_{x y}:(x, y) \in \mathcal{E}\right\rangle
$$

of atoms is a semi-scaffold, by Lemma 6.3 .

It is worth pointing out that, in general, it is not true that every atomic, measurable relation algebra has a scaffold.

Fix a semi-scaffold

$$
a=\left\langle a_{x y}:(x, y) \in \mathcal{E}\right\rangle
$$

in $\mathfrak{A}$, and consider an arbitrary atom $b=a_{x y}$ in this semi-scaffold. The right and left stabilizers $H_{b}$ and $K_{b}$ are normal subgroups of the groups $G_{x}$ and $G_{y}$ of permutations of $x$ and $y$ respectively, by Corollary 4.16. Write

$$
H_{x y}=H_{b} \quad \text { and } \quad K_{x y}=K_{b} .
$$


The quotient groups $G_{x} / H_{x y}$ and $G_{y} / K_{x y}$ are isomorphic, and in fact there are cosets systems

$$
\left\langle H_{x y, \xi}: \xi<\kappa_{x y}\right\rangle \quad \text { and } \quad\left\langle K_{x y, \xi}: \xi<\kappa_{x y}\right\rangle
$$

of $H_{x y}$ and $K_{x y}$ in $G_{x}$ and $G_{y}$ respectively such that

$$
H_{x y, \xi} ; a_{x y}=a_{x y} ; K_{x y, \xi},
$$

and the function $\varphi_{x y}$ from $G_{x} / H_{x y}$ to $G_{y} / K_{x y}$ defined by

$$
\varphi_{x y}\left(H_{x y, \xi}\right)=K_{x y, \xi}
$$

for each $\xi$ is an isomorphism, by Isomorphism Theorem 5.4 and the subsequent remarks. Without loss of generality, it may be assumed that

$$
H_{x y, 0}=H_{x y} \quad \text { and } \quad K_{x y, 0}=K_{x y} .
$$

The left stabilizer $H_{b} \smile$ coincides with $K_{b}$, the right stabilizer $K_{b} \smile$ coincides with $H_{b}$, and the quotient isomorphism $\varphi_{b} \smile$ coincides with $\varphi_{b}^{-1}$, by Converse Theorem 5.11 (with $b$ in place of $a$ ). Consequently, since

$$
a_{y x}=a_{x y}^{\smile}=b^{\smile},
$$

by semi-scaffold condition (ii) and the definition of $b$, we have

$$
H_{y x}=K_{x y} \quad \text { and } \quad K_{y x}=H_{x y} .
$$

Apply Convention 5.12 to choose coset systems

$$
\left\langle H_{y x, \xi}: \xi<\kappa_{x y}\right\rangle \quad \text { and } \quad\left\langle K_{y x, \xi}: \xi<\kappa_{x y}\right\rangle
$$

of $H_{y x}$ and $K_{y x}$ in $G_{y}$ and $G_{x}$ respectively such that

$$
H_{y x, \xi}=K_{x y, \xi} \quad \text { and } \quad K_{y x, \xi}=H_{x y, \xi}
$$

for each $\xi<\kappa_{x y}$, and the function $\varphi_{y x}$ from $G_{y} / H_{y x}$ to $G_{x} / K_{y x}$ that is the inverse of $\varphi_{x y}$ is determined by

For each $\alpha<\kappa_{x y}$, the element

$$
\varphi_{y x}\left(H_{y x, \xi}\right)=K_{y x, \xi}
$$

$$
a_{x y, \alpha}=H_{x y, \alpha} ; a_{x y}=a_{x y} ; K_{x y, \alpha}
$$

is well defined, by Corollary 3.10, and the system of elements $\left\langle a_{x y, \alpha}: \alpha<\kappa_{x y}\right\rangle$ is a partition of $x ; 1 ; y$ into atoms, by Atomic Partition Lemma 4.11. The following lemma summarizes these observations.

Lemma 6.5. Let $(x, y)$ be a pair in $\mathcal{E}$. The system of elements $\left\langle a_{x y, \alpha}: \alpha<\kappa_{x y}\right\rangle$ is an atomic partition of $x ; 1 ; y$. Consequently, an element below $x ; 1 ; y$ is an atom if and only if it has the form $a_{x y, \alpha}$ for a (unique) $\alpha<\kappa_{x y}$.

The preceding lemma is local in character, referring to atoms below a given rectangle with measurable sides. The lemma implies a corresponding global result.

Lemma 6.6 (Semi-scaffold Partition Lemma). The system of elements

$$
\left\langle a_{x y, \alpha}:(x, y) \in \mathcal{E} \text { and } \alpha<\kappa_{x y}\right\rangle
$$

is an atomic partition of the unit. Consequently, an element in the algebra is an atom if and only if it has the form $a_{x y, \alpha}$ for some (unique) pair $(x, y)$ in $\mathcal{E}$ and $a$ (unique) $\alpha<\kappa_{x y}$. 
Proof. The given relation algebra is assumed to be measurable, so

$$
1^{\prime}=\sum I,
$$

by the definition of measurability. Use the identity laws, (1), complete distributivity, and definition of $\mathcal{E}$ as the set of pairs $(x, y)$ such that $x ; 1 ; y \neq 0$ to obtain

$$
\begin{aligned}
1=1^{\prime} ; 1 ; 1^{\prime}=\left(\sum I\right) & ; 1 ;\left(\sum I\right) \\
& =\sum\{x ; 1 ; y: x, y \in I\}=\sum\{x ; 1 ; y:(x, y) \in \mathcal{E}\} .
\end{aligned}
$$

For each pair $(x, y)$ in $\mathcal{E}$, we have

$$
x ; 1 ; y=\sum\left\{a_{x y, \alpha}: \alpha<\kappa_{x y}\right\},
$$

by Lemma 6.5 . Combine (2) and (3) to arrive at

$$
1=\sum\left\{a_{x y, \alpha}:(x, y) \in \mathcal{E} \text { and } \alpha<\kappa_{x y}\right\} .
$$

It remains to prove that two atoms $a_{x y, \alpha}$ and $a_{u v, \beta}$ are disjoint if $x \neq u$, or $y \neq v$, or $x=y$ and $u=v$, but $\alpha \neq \beta$. In the first case, we have

$$
a_{x y, \alpha} \cdot a_{u v, \beta} \leq(x ; 1 ; y) \cdot(u ; 1 ; v)=(x \cdot u) ; 1 ;(y \cdot v)=0 ; 1 ;(y \cdot v)=0,
$$

by monotony, the assumption that $\left\langle a_{x y}:(x, y) \in \mathcal{E}\right\rangle$ is a semi-scaffold, Lemma 2.5 (ii), the assumption that $x$ and $u$ are distinct atoms, and Lemma 2.2(i). A similar argument applies in the second case, when $y \neq v$. In the third case, the desired disjointness follows directly from Lemma 6.5.

The next lemma specifies the elements $a_{x y, \alpha}$ that are subidentity atoms

Lemma 6.7 (Semi-scaffold Identity Lemma). An element $a_{x y, \alpha}$ is a subidentity atom if and only if $x=y$ and $\alpha=0$. Consequently,

$$
1^{\prime}=\sum\left\{a_{x x, 0}: x \in I\right\} .
$$

Proof. The rectangles $x ; 1 ; y$ have a non-zero meet with the identity element 1 ' if and only if $x=y$, and in this case that meet is $x$, by Lemma 2.5(i) and the assumption that $x$ and $y$ are atoms. This means that the atom $a_{x y, \alpha}$, which is below the rectangle $x ; 1 ; y$, by Lemma 6.5 , can lie below 1' only when $x=y$ and

$$
a_{x x, \alpha}=x=a_{x x} .
$$

For this last equality to hold, the index $\alpha$ must be 0 , by the definition of $a_{x y, \alpha}$ and the assumption about the indexing of the coset systems. This proves the first assertion of the lemma. The second follows from the first and the assumption that the relation algebra is measurable.

The next lemma determines the converse of each atom $a_{x y, \alpha}$.

Lemma 6.8 (Semi-scaffold Converse Lemma). For each pair $(x, y)$ in $\mathcal{E}$, and each $\alpha<\kappa_{x y}$, we have $a_{x y, \alpha}^{\smile}=a_{y x, \beta}$, where $H_{x y, \alpha}^{\smile}=H_{x y, \beta}$.

Proof. Assume

$$
H_{x y, \alpha}^{\smile}=H_{x y, \beta} .
$$

Use the definition of $a_{x y, \alpha}$, the second involution law, semi-scaffold condition (iii) and (1), the assumption about the system of cosets $K_{y x, \beta}$, described after Lemma 6.4 
and based on Convention 5.12, and Convention 5.5 and the definition of $a_{y x, \beta}$ to arrive at

$$
\begin{aligned}
\left.\left(a_{x y, \alpha}\right)^{\smile=(} H_{x y, \alpha} ; a_{x y}\right)^{\smile}=a_{x y}^{\smile} ; H_{x y, \alpha}^{\smile} & =a_{y x} ; H_{x y, \beta} \\
& =a_{y x} ; K_{y x, \beta}=H_{y x, \beta} ; a_{y x}=a_{y x, \beta} .
\end{aligned}
$$

The next step is to determine the relative product of two atoms $a_{x y, \alpha}$ and $a_{w z, \beta}$. Begin with the trivial case.

Lemma 6.9. Let $(x, y)$ and $(w, z)$ be pairs in $\mathcal{E}$, and $\alpha<\kappa_{x y}$ and $\beta<\kappa_{w z}$. If $y \neq w$, then $a_{x y, \alpha} ; a_{w z, \beta}=0$.

Proof. If $y$ and $w$ are not equal, then they are disjoint, because they are atoms, and therefore

$$
a_{x y, \alpha} ; a_{w z, \beta} \leq(x ; 1 ; y) ;(w ; 1 ; z)=0,
$$

by semi-scaffold condition (i), monotony, and Lemma $2.5(\mathrm{v})$.

The non-trivial case of relative multiplication is more interesting and more involved.

Lemma 6.10 (Semi-scaffold Relative Product Lemma). Let $(x, y)$ and $(y, z)$ be pairs in $\mathcal{E}$, and $\alpha<\kappa_{x y}$ and $\beta<\kappa_{y z}$. If $\xi<\kappa_{x z}$ is any index such that

$$
H_{x z, \xi} ; a_{x z} \leq a_{x y} ; a_{y z},
$$

then

$$
a_{x y, \alpha} ; a_{y z, \beta}=\sum\left\{a_{x z, \gamma}: \gamma<\kappa_{x z} \text { and } H_{x z, \gamma} \subseteq \varphi_{x y}^{-1}\left[K_{x y, \alpha} ; H_{y z, \beta}\right] ; H_{x z, \xi}\right\} .
$$

Proof. The relative product $a_{x y} ; a_{y z}$ is a regular element below $x ; 1 ; z$ with normal stabilizers, by Relative Product Theorem 5.14, and the left stabilizer is the product group $H_{x y} ; H_{x z}$, by Image Theorem 5.18 (with $(x, y),(y, z)$, and $(x, z)$ in place of $a, b$, and $c$ respectively). The isomorphism $\varphi_{x y}$ from $G_{x} / H_{x y}$ to $G_{y} / K_{x y}$ induces an isomorphism $\hat{\varphi}_{x y}$ from

$$
G_{x} /\left(H_{x y} ; H_{x z}\right) \quad \text { to } \quad G_{y} /\left(K_{x y} ; H_{y z}\right)
$$

by Image Theorem 5.18 . To simplify notation, write

$$
M=H_{x y} ; H_{x z} \quad \text { and } \quad P=K_{x y} ; H_{y z},
$$

so that $M$ is the left stabilizer of $a_{x y} ; a_{y z}$, and $\hat{\varphi}_{x y}$ maps $G_{x} / M$ isomorphically to $G_{y} / P$. Let

$$
\left\langle M_{\eta}: \eta<\lambda\right\rangle \quad \text { and } \quad\left\langle P_{\eta}: \eta<\lambda\right\rangle
$$

be coset systems of $M$ and $P$ in $G_{x}$ and $G_{y}$ respectively such that

$$
\varphi_{x y}\left[M_{\eta}\right]=P_{\eta}
$$

for each $\eta<\lambda$. The coset product $K_{x y, \alpha} ; H_{y z, \beta}$ is a coset of $P$, so it must coincide with $P_{\rho}$ for some $\rho<\lambda$, and therefore

$$
M_{\rho}=\varphi_{x y}^{-1}\left[P_{\rho}\right]=\varphi_{x y}^{-1}\left[K_{x y, \alpha} ; H_{y z, \beta}\right] .
$$

The principal step in the proof is showing that

$$
M_{\rho} ; a_{x y} ; a_{y z}=a_{x y, \alpha} ; a_{y z, \beta} .
$$


As $H_{x y}$ is a subgroup of $M$, there must be a partition $\left\langle\Gamma_{\eta}: \eta<\lambda\right\rangle$ of the index set $\kappa_{x y}$ such that

$$
M_{\eta}=\bigcup_{\zeta \in \Gamma_{\eta}} H_{x y, \zeta}
$$

for each $\eta$. Taking $\rho$ for $\eta$ gives

$$
\begin{aligned}
K_{x y, \alpha} ; H_{y z, \beta}=\varphi_{x y}\left[M_{\rho}\right]=\varphi_{x y}\left[\bigcup_{\zeta \in \Gamma_{\rho}}\right. & \left.H_{x y, \zeta}\right] \\
& =\bigcup_{\zeta \in \Gamma_{\rho}} \varphi_{x y}\left(H_{x y, \zeta}\right)=\bigcup_{\zeta \in \Gamma_{\rho}} K_{x y, \zeta},
\end{aligned}
$$

by (1), (3), the distributivity of function images over unions, and the definition of $\varphi_{x y}$. Recall from the remarks preceding Lemma 6.5 that

$$
H_{x y, \zeta} ; a_{x y}=a_{x y} ; K_{x y, \zeta} .
$$

for each $\zeta<\kappa_{x y}$. Compute:

$$
\begin{aligned}
M_{\rho} ; a_{x y} ; a_{y z} & =\left(\bigcup_{\zeta \in \Gamma_{\rho}} H_{x y, \zeta}\right) ; a_{x y} ; a_{y z}=\sum_{\zeta \in \Gamma_{\rho}} H_{x y, \zeta} ; a_{x y} ; a_{y z} \\
& =\sum_{\zeta \in \Gamma_{\rho}} a_{x y} ; K_{x y, \zeta} ; a_{y z}=a_{x y} ;\left(\bigcup_{\zeta \in \Gamma_{\rho}} K_{x y, \zeta}\right) ; a_{y z} \\
& =a_{x y} ;\left(K_{x y, \alpha} ; H_{y z, \beta}\right) ; a_{y z}=\left(a_{x y} ; K_{x y, \alpha}\right) ;\left(H_{y z, \beta} ; a_{y z}\right) \\
& =a_{x y, \alpha} ; a_{y z, \beta},
\end{aligned}
$$

by (3), complete distributivity, (5), complete distributivity, (4), associativity, and the definitions of $a_{x y, \alpha}$ and $a_{y z, \beta}$. This proves (2).

The group $H_{x z}$ is included in $M$, by the definition of $M$, so there is a partition $\left\langle\Delta_{\eta}: \eta<\lambda\right\rangle$ of $\kappa_{x z}$ such that

$$
M_{\eta}=\bigcup_{\gamma \in \Delta_{\eta}} H_{x z, \gamma}
$$

for each $\eta<\lambda$. Let $\xi<\kappa_{x z}$ be an index such that

$$
H_{x z, \xi} ; a_{x z} \leq a_{x y} ; a_{y z} .
$$

There is a unique index $\sigma<\mu$ such that

$$
M_{\sigma}=M_{\rho} ; H_{x z, \xi},
$$

and for this index we have

$$
\bigcup_{\gamma \in \Delta_{\sigma}} H_{x z, \gamma}=M_{\sigma}=M_{\rho} ; H_{x z, \xi}=\varphi_{x y}^{-1}\left[K_{x y, \alpha} ; K_{y z, \beta}\right] ; H_{x z, \xi}
$$

by (6) (with $\sigma$ in place of $\eta$ ), (8), , and (1). Thus, $\Delta_{\sigma}$ is precisely the set of indices $\gamma$ such that

$$
H_{x z, \gamma} \subseteq \varphi_{x y}^{-1}\left(K_{x y, \alpha} ; K_{y z, \beta}\right) ; H_{x z, \xi} \quad \text { if and only if } \quad \gamma \in \Delta_{\sigma} .
$$

Use assumption (7) for the first and only time, Partition Lemma 4.12 (with $M$, $\rho, a_{x y} ; a_{y z}$, and $H_{x z, \xi} ; a_{x z}$ in place of $H_{b}, \eta, b$, and $a$ respectively), and (8), to arrive at

$$
M_{\rho} ; a_{x y} ; a_{y z}=\sum M_{\rho} ; H_{x z, \xi} ; a_{x z}=\sum M_{\sigma} ; a_{x z} .
$$

Conclude that

$$
\begin{aligned}
& a_{x y, \alpha} ; a_{y z, \beta}=M_{\rho} ; a_{x y} ; a_{y z}=\sum M_{\sigma} ; a_{x z} \\
& \quad=\sum\left(\bigcup_{\gamma \in \Delta_{\sigma}} H_{x z, \gamma}\right) ; a_{x z}=\sum_{\gamma \in \Delta_{\sigma}} H_{x z, \gamma} ; a_{x z}=\sum_{\gamma \in \Delta_{\sigma}} a_{x z, \gamma}
\end{aligned}
$$

by (2), (10), (6) (with $\sigma$ in place of $\eta$ ), complete distributivity, and the definition of $a_{x z, \gamma}$. In view of (9), the equality of the first and last terms in (11) is just what was to be shown. 
The formula in Lemma 6.10 for computing the relative product $a_{x y, \alpha} ; a_{y z, \beta}$ takes on a more familiar form when the system $a$ is actually a scaffold, and not just a semi-scaffold.

Lemma 6.11 (Scaffold Relative Product Lemma). Let $(x, y)$ and $(y, z)$ be pairs in $\mathcal{E}$, and $\alpha<\kappa_{x y}$ and $\beta<\kappa_{y z}$. If $a$ is a scaffold, then

$$
a_{x y, \alpha} ; a_{y z, \beta}=\sum\left\{a_{x z, \gamma}: \gamma<\kappa_{x z} \text { and } H_{x z, \gamma} \subseteq \varphi_{x y}^{-1}\left[K_{x y, \alpha} ; H_{y z, \beta}\right]\right\} .
$$

Proof. The assumption that $a$ is a scaffold implies that $a_{x z} \leq a_{x y} ; a_{y z}$. Consequently, the index $\xi<\kappa_{x z}$ in the statement of Lemma 6.10 may be taken to be 0 . The term $\varphi_{x y}^{-1}\left[K_{x y, \alpha} ; H_{y z, \beta}\right]$ in the formula for computing the relative product $a_{x y, \alpha} ; a_{y z, \beta}$ is a coset of the product group

$$
M=H_{x y} ; H_{x z},
$$

which is the identity element of the quotient group $G_{z} / M$. Forming the relative product of this term on the right by the subset $H_{x z, 0}=H_{x z}$ of $M$ does not change it:

$$
\varphi_{x y}^{-1}\left[K_{x y, \alpha} ; H_{y z, \beta}\right] ; H_{x z, 0}=\varphi_{x y}^{-1}\left[K_{x y, \alpha} ; H_{y z, \beta}\right] .
$$

Thus, the formula for computing the relative product $a_{x y, \alpha} ; a_{y z, \beta}$ that is given in Lemma 6.10 reduces in this case to the formula that is given in the statement of the present lemma.

\section{REPRESENTATION THEOREMS}

This section contains the main result of the paper, a representation theorem for atomic, measurable relation algebras. Continue with the assumption that $\mathfrak{A}$ is a complete and atomic, measurable relation algebra. Here is a summary of what has been accomplished so far in the analysis of the structure of $\mathfrak{A}$.

In Definition 3.4, a system of mutually disjoint groups

$$
G=\left\langle G_{x}: x \in I\right\rangle
$$

indexed by the set $I$ of measurable atoms in $\mathfrak{A}$ is defined, where $G_{x}$ is the group of permutations in $\mathfrak{A}$ of the measurable atom $x$ (see Lemmas 3.3 and 3.6). After Definition 6.1 , an equivalence relation $\mathcal{E}$ is defined on the set $I$ by putting a pair $(x, y)$ in $\mathcal{E}$ just in case the rectangle $x ; 1 ; y$ is not zero. It is shown in Semi-scaffold Existence Lemma 6.4 that $\mathfrak{A}$ has a semi-scaffold

$$
a=\left\langle a_{x y}:(x, y) \in \mathcal{E}\right\rangle .
$$

Fix such a semi-scaffold $a$ for the remainder of the discussion.

In terms of $a$, for each pair $(x, y)$ in $\mathcal{E}$, normal subgroups $H_{x y}$ and $K_{x y}$ of $G_{x}$ and $G_{y}$ respectively are defined after Semi-scaffold Existence Lemma 6.4 as the left and right stabilizers of the atom $a_{x y}$. With the help of Isomorphism Theorem 5.4, it is shown that there is a canonical quotient isomorphism $\varphi_{x y}$ from $G_{x} / H_{x y}$ to $G_{y} / K_{x y}$. In fact, there are coset systems

$$
\left\langle H_{x y, \xi}: \xi<\kappa_{x y}\right\rangle \quad \text { and } \quad\left\langle K_{x y, \xi}: \xi<\kappa_{x y}\right\rangle
$$


such that

$$
H_{x y, \xi} ; a_{x y}=a_{x y} ; K_{x y, \xi}
$$

for each $\xi$, and the isomorphism $\varphi_{x y}$ maps $H_{x y, \xi}$ to $K_{x y, \xi}$ for each $\xi$. The system of isomorphisms

$$
\varphi=\left\langle\varphi_{x y}:(x, y) \in \mathcal{E}\right\rangle
$$

has the following properties. First, for each $x$ in $I$, the atom $a_{x x}$ coincides with $x$ by semi-scaffold condition (ii), so the isomorphism $\varphi_{x x}$ is the identity automorphism of $G_{x} /\{x\}$, by Identity Theorem 5.10. Second, for each pair $(x, y)$ in $\mathcal{E}$, the atom $a_{y x}$ coincides with the converse $a_{x y}^{\smile}$, by semi-scaffold condition (iii), so the isomorphism $\varphi_{y x}$ is the inverse of the isomorphism $\varphi_{x y}$, by Converse Theorem 5.11. In fact,

$$
H_{y x}=K_{x y} \quad \text { and } \quad K_{y x}=H_{x y}
$$

and we may choose cosets systems

$$
\left\langle H_{y x, \xi}: \xi<\kappa_{x y}\right\rangle \text { and }\left\langle K_{y x, \xi}: \xi<\kappa_{x y}\right\rangle
$$

of $H_{y x}$ and $K_{y x}$ in $G_{y}$ and $G_{x}$ respectively such that

$$
H_{y x, \xi}=K_{x y, \xi} \quad \text { and } \quad K_{y x, \xi}=H_{x y, \xi},
$$

so that $\varphi_{y x}$ maps $K_{x y, \xi}$ to $H_{x y, \xi}$ for each $\xi$.

Third, for every triple $(x, y, z)$ in the set

$$
\mathcal{E}_{3}=\{(x, y, z):(x, y) \text { and }(y, z) \in \mathcal{E}\},
$$

the atoms $a_{x y}, a_{y z}$, and $a_{x z}$ satisfy the inequalities

$$
a_{x y} \leq x ; 1 ; y, \quad a_{y z} \leq y ; 1 ; z, \quad \text { and } \quad a_{x z} \leq x ; 1 ; z,
$$

by semi-scaffold condition (i), and therefore

$$
\varphi_{x y}\left[H_{x y} ; H_{x z}\right]=K_{x y} ; H_{y z}
$$

by Image Theorem 5.18. The preceding observations combine to show that conditions (i)-(iii) in Definition 5.3 of a coset semi-frame are satisfied.

Turn now to the task of defining a coset system

$$
C=\left\langle C_{x y z}:(x, y, z) \in \mathcal{E}_{3}\right\rangle
$$

such that condition (iv) in Definition 5.3 is satisfied. For each triple $(x, y, z)$ in $\mathcal{E}_{3}$ the relative product $a_{x y} ; a_{y z}$ is a regular element below $x ; 1 ; z$ with normal stabilizers, by Relative Product Theorem 5.14. In particular, this relative product is not zero, by Lemma 4.4. The atom $a_{x z}$ is also below $x ; 1 ; z$, by semi-scaffold condition (i), so there must be a coset $H_{x z, \zeta}$ of $H_{x z}$ (with $\zeta<\kappa_{x z}$ ) such that

$$
a_{x z, \zeta}=H_{x z, \zeta} ; a_{x z} \leq a_{x y} ; a_{y z},
$$

by Lemma 6.5 (with $z$ in place of $y$ ). Notice in passing that Lemma 6.5 is an easy consequence of Atomic Partition Lemma 4.11. Choose any such index $\zeta<\kappa_{x z}$, and write

$$
C_{x y z}=H_{x y} ; H_{x z, \zeta} .
$$

Observe that $C_{x y z}$ is a coset of the product group $H_{x y} ; H_{x z}$ in $G_{x}$. The coset $H_{x z, \zeta}$ determines an inner automorphism $\tau$ of $G_{z} / H_{x z}$ that is defined by

$$
\tau\left(H_{x z, \eta}\right)=H_{x z, \zeta}^{\smile} ; H_{x z, \eta} ; H_{x z, \zeta}
$$


for each $\eta<\kappa_{x z}$. In turn, $\tau$ induces an inner automorphism $\hat{\tau}$ of $G_{z} /\left(H_{x y} ; H_{x z}\right)$ that is defined as follows. Write a given $\operatorname{coset} H_{x y, \alpha} ; H_{x z, \beta}$ of $H_{x y} ; H_{x z}$ as a union

$$
H_{x y, \alpha} ; H_{x z, \beta}=\bigcup\left\{H_{x z, \eta}: \eta \in \Gamma\right\}
$$

of cosets of $H_{x z}$, where $\Gamma$ is some subset of the index set $\kappa_{x z}$, and define $\hat{\tau}$ by

(6) $\hat{\tau}\left(H_{x y, \alpha} ; H_{x z, \beta}\right)=\bigcup\left\{\tau\left(H_{x z, \eta}\right): \eta \in \Gamma\right\}=\bigcup\left\{H_{x z, \zeta}^{\smile} ; H_{x z, \eta} ; H_{x z, \zeta}: \eta \in \Gamma\right\}$.

The definition of $C_{x y z}$ in (5), the second involution law, complete distributivity, and the fact that $H_{x z}$ is the identity coset of the quotient group $G_{x} / H_{x z}$ together imply that

$$
\begin{aligned}
& C_{x y z}^{\smile} ;\left(H_{x y, \alpha} ; H_{x z, \beta}\right) ; C_{x y z} \\
&=\left(H_{x z} ; H_{x z, \zeta}\right)^{\smile} ;\left(\bigcup\left\{H_{x z, \eta}: \eta \in \Gamma\right\}\right) ;\left(H_{x z} ; H_{x z, \zeta}\right) \\
&=\bigcup\left\{H_{x z, \zeta}^{\smile} ; H_{x z}^{\smile} ; H_{x z, \eta} ; H_{x z} ; H_{x z, \zeta}: \eta \in \Gamma\right\} \\
& \quad=\bigcup\left\{H_{x z, \zeta}^{\smile} ; H_{x z, \eta} ; H_{x z, \zeta}: \eta \in \Gamma\right\} .
\end{aligned}
$$

Compare (6) with (7) to see that the inner automorphism $\hat{\tau}$ of $G_{x} /\left(H_{x y} ; H_{x z}\right)$ induced by $\tau$, that is to say, induced by the coset $H_{x z, \zeta}$, coincides with the inner automorphism determined by $C_{x y z}$.

The index $\zeta$ was chosen so that (4) is satisfied. Apply Relative Product Theorem 5.15 (with $\zeta$ in place of $\xi$ ) to conclude that

$$
\hat{\varphi}_{x y}\left|\hat{\varphi}_{y z}=\hat{\tau}\right| \hat{\varphi}_{x z} .
$$

The remarks of the previous paragraph show that $\hat{\tau}$ coincides with the inner automorphism of $G_{x} /\left(H_{x y} ; H_{x z}\right)$ determined by the coset $C_{x y z}$. Consequently, semiframe condition (iv) is satisfied.

Take $C$ to be the system of cosets defined in (3) and (5), and form the group triple

$$
\mathcal{F}=(G, \varphi, C) .
$$

The following theorem about $\mathcal{F}$ has been proved.

Theorem 7.1 (Semi-frame Theorem). The group triple $\mathcal{F}$ is a coset semi-frame.

We shall refer to $\mathcal{F}$ as the semi-frame associated with the semi-scaffold $a$.

It may appear as if the semi-frame depends not only on the particular semiscaffold $a$ that has been selected, but also on the particular coset that is chosen to satisfy (4). However, it is not difficult to see that this is in fact not the case. If $H_{x z, \zeta^{\prime}}$ is any other coset such that (4) (with $\zeta^{\prime}$ in place of $\zeta$ ) holds, then

$$
H_{x y} ; H_{x z, \zeta^{\prime}}=H_{x y} ; H_{x z, \zeta}=C_{x y z} .
$$

In more detail, the product subgroup $H_{x y} ; H_{x z}$ is the left stabilizer of the relative product $a_{x y} ; a_{y z}$, by Relative Product Theorem 5.14 and Image Theorem 5.18. In particular, it leaves this relative product fixed under relative multiplication on the left. Use this fact (at the end of the computation), together with the identity property of $H_{x z}$ in the quotient group $G_{x} / H_{x z}$, (4), and Partition Lemma 4.12 (with

$$
H_{x z, \zeta} ; a_{x z}, \quad a_{x y} ; a_{y z}, \quad \text { and } \quad H_{x y} ; H_{x z}
$$


in place of $a, b$, and $H_{b, \eta}$ respectively), to obtain

$$
\begin{aligned}
\sum H_{x y} ; H_{x z, \zeta} ; a_{x z}=\sum H_{x y} ; H_{x z} ; H_{x z, \zeta} & ; a_{x z} \\
& =H_{x y} ; H_{x z} ; a_{x y} ; a_{y z}=a_{x y} ; a_{y z} .
\end{aligned}
$$

A similar argument shows that

$$
\sum H_{x y} ; H_{x z, \zeta^{\prime}} ; a_{x z}=a_{x y} ; a_{y z},
$$

so

$$
\sum H_{x y} ; H_{x z, \zeta} ; a_{x z}=\sum H_{x y} ; H_{x z, \zeta^{\prime}} ; a_{x z} .
$$

Use Corollary 4.10 to conclude that the two cosets $H_{x y} ; H_{x z, \zeta}$ and $H_{x y} ; H_{x z, \zeta^{\prime}}$ are equal.

In terms of the semi-frame $\mathcal{F}$, a complete and atomic Boolean algebra with additional completely distributive operations, that is to say, a complete and atomic Boolean algebra with complete operators

$$
\mathfrak{C}[\mathcal{F}]=\left\langle C[\mathcal{F}], \cup, \sim, \otimes,{ }^{-1}, i d_{U}\right\rangle
$$

of the same similarity type as relation algebras, can be defined. The atoms of this algebra are the binary relations $R_{x y, \alpha}$ that are the subsets of the Cartesian product $G_{x} \times G_{y}$ defined by

$$
R_{x y, \alpha}=\bigcup\left\{H_{x y, \xi} \times\left(K_{x y, \xi} ; K_{x y, \alpha}\right): \xi<\kappa_{x y}\right\}
$$

for each pair $(x, y)$ in $\mathcal{E}$ and each $\alpha<\kappa_{x y}$. In particular, for $x=y$, the left and right stabilizers are the trivial subgroups

$$
H_{x y}=\{x\} \quad \text { and } \quad K_{x y}=\{y\},
$$

and the cosets are the singletons of elements of the respective groups, by semi-frame condition (i), so that the definition of $R_{x x, \alpha}$ assumes the form

$$
R_{x x, \alpha}=\left\{\left(g, g ; g_{\alpha}\right): g \in G_{x}\right\},
$$

where $K_{x x, \alpha}=\left\{g_{\alpha}\right\}$. When $\alpha=0$, the coset $K_{x x, \alpha}$ coincides with the trivial subgroup $K_{0}=\{x\}$, by convention, so that $g_{0}=x$, and therefore $R_{x x, 0}$ is the identity relation on the set $G_{x}$.

The elements of the algebra are arbitrary unions of sets of atoms, so the universe of the algebra is a set of binary relations on the base set

$$
U=\bigcup\left\{G_{x}: x \in I\right\} .
$$

The Boolean operations of the algebra are the binary set-theoretic operation $U$ of forming unions of binary relations, and the unary set-theoretic operation of forming complements of binary relations with respect to the unit, or universal, relation $U \times U$, which is the union of the set of atoms. The distinguished constant $i d_{U}$ is the identity relation on $U$, and

$$
i d_{U}=\bigcup\left\{R_{x x, 0}: x \in I\right\} .
$$

The operation ${ }^{-1}$ is the unary set-theoretic operation on binary relations of forming the converse, or inverse, of a relation. Semi-frame condition (ii) in Definition 5.3 implies that this operation is determined on atoms by

$$
R_{x y, \alpha}^{-1}=R_{y x, \beta}, \quad \text { where } \quad H_{x y, \alpha}^{\smile}=H_{x y, \beta},
$$


that is to say, where $H_{x y, \beta}$ is the coset inverse of $H_{x y, \alpha}$ in the quotient group $G_{x} / H_{x y}$, and the operation is extended to all elements in the algebra by making it completely distributive over unions.

The binary operation $\otimes$ is defined on atoms as follows. For pairs $(x, y)$ and $(w, z)$ in $\mathcal{E}$ with $y \neq w$,

$$
R_{x y, \alpha} \otimes R_{w z, \beta}=\varnothing
$$

for all $\alpha<\kappa_{x y}$ and $\beta<\kappa_{w z}$, and for pairs $(x, y)$ and $(y, z)$ in $\mathcal{E}$,

$$
R_{x y, \alpha} \otimes R_{y z, \beta}=\bigcup\left\{R_{x z, \gamma}: H_{x z, \gamma} \subseteq \varphi_{x y}^{-1}\left[K_{x y, \alpha} ; H_{y z, \beta}\right] ; C_{x y z}\right\}
$$

for all $\alpha<\kappa_{x y}$ and all $\beta<\kappa_{y z}$. The operation is extended to arbitrary pairs of elements in $\mathfrak{C}[\mathcal{F}]$ by making it be completely distributive over arbitrary unions.

The algebra $\mathfrak{C}[\mathcal{F}]$ turns out to be a relation algebra if and only if certain conditions called the coset conditions are satisfied, and in this case $\mathfrak{C}[\mathcal{F}]$ is called a coset relation algebra. The coset conditions do not play a role in the discussion below, so we do not go into them further (see [1]).

In the proof of the representation theorem for atomic, measurable relation algebras, we shall use a form of the Atomic Isomorphism Theorem (see [3]). The hypothesis of this form of the theorem is that two complete and atomic Boolean algebras with completely distributive operators are given, say $\mathfrak{A}$ and $\mathfrak{B}$, and say of the same similarity type as relation algebras, together with a bijection $\vartheta$ from the set of atoms in $\mathfrak{A}$ to the set of atoms in $\mathfrak{B}$. Using relation algebraic notation for the operations, the conclusion of the theorem may be formulated as follows. The bijection $\vartheta$ can be extended to an isomorphism from $\mathfrak{A}$ to $\mathfrak{B}$ if and only if $\vartheta$ preserves the Peircean operations on atoms in the sense that

$$
\begin{array}{lll}
c \leq a ; b & \text { if and only if } & \vartheta(c) \leq \vartheta(a) ; \vartheta(b), \\
c \leq a^{-} & \text {if and only if } & \vartheta(c) \leq \vartheta(a)^{-}, \\
c \leq 1 & \text { if and only if } & \vartheta(c) \leq 1,
\end{array}
$$

for all atoms $a, b$, and $c$ in $\mathfrak{A}$, where the operations on the left (including the operation 1 ' of rank 0 ) are those of $\mathfrak{A}$, and the ones on the right are those of $\mathfrak{B}$. If these conditions are satisfied, then the isomorphism from $\mathfrak{A}$ to $\mathfrak{B}$ is the function $\psi$ defined by

$$
\psi(r)=\sum\{\vartheta(a): a \in X\}
$$

for every element $r$ in $\mathfrak{A}$, where $X$ is the set of atoms in $\mathfrak{A}$ that are below $r$.

The next theorem says that coset relation algebras are essentially the only possible examples of atomic, measurable relation algebras.

Theorem 7.2 (Representation Theorem). Every atomic, measurable relation algebra is essentially isomorphic to a coset relation algebra.

Proof. Start with an atomic, measurable relation algebra $\mathfrak{B}$, and pass to its completion $\mathfrak{A}$, that is to say, pass to its minimal complete extension. The completion $\mathfrak{A}$ is well known to be a complete and atomic relation algebra, and its atoms are the same as the atoms in $\mathfrak{B}$ (see [11]). It follows that each subidentity atom $x$ is measurable not only in $\mathfrak{B}$, but also in $\mathfrak{A}$, because the same atoms are below the rectangle $x ; 1 ; x$ in both $\mathfrak{B}$ and $\mathfrak{A}$, and consequently $\mathfrak{A}$ is a complete and atomic, measurable relation algebra. 
Let $I$ be the set of measurable atoms in $\mathfrak{A}$, and $\mathcal{E}$ the equivalence relation defined on $I$ by putting $(x, y)$ in $\mathcal{E}$ if and only if $x ; 1 ; y \neq 0$. The algebra $\mathfrak{A}$ has a semiscaffold, by Semi-scaffold Existence Lemma 6.4. Fix such a semi-scaffold

$$
a=\left\langle a_{x y}:(x, y) \in \mathcal{E}\right\rangle
$$

and let

$$
\mathcal{F}=(G, \varphi, C)
$$

be the coset semi-frame associated with $a$, where

$$
G=\left\langle G_{x}: x \in I\right\rangle, \quad \varphi=\left\langle\varphi_{x y}:(x, y) \in \mathcal{E}\right\rangle, \quad C=\left\langle C_{x y z}:(x, y, z) \in \mathcal{E}_{3}\right\rangle
$$

are as defined at the beginning of the section. (Here, Semi-frame Theorem 7.1 is being used.) The goal is to show that $\mathfrak{A}$ is isomorphic to $\mathfrak{C}[\mathcal{F}]$. It then follows that $\mathfrak{C}[\mathcal{F}]$ is a relation algebra, and therefore it automatically satisfies the coset conditions. Conclusion: $\mathfrak{A}$ is isomorphic to a coset relation algebra, so $\mathfrak{B}$ is essentially isomorphic to a coset relation algebra.

The distinct atoms in $\mathfrak{A}$ are the elements $a_{x y, \alpha}$ defined before Lemma 6.5 , by Semi-scaffold Partition Lemma 6.6. The distinct atoms in $\mathfrak{C}[\mathcal{F}]$ are the binary relations $R_{x y, \alpha}$ defined after Semi-frame Theorem 7.1. Let $\vartheta$ be the bijection from the set of atoms in $\mathfrak{A}$ to the set of atoms in $\mathfrak{C}[\mathcal{F}]$ that is defined by

$$
\vartheta\left(a_{x y, \alpha}\right)=R_{x y, \alpha}
$$

for every pair $(x, y)$ in $\mathcal{E}$ and every $\alpha<\kappa_{x y}$. It must be shown that $\vartheta$ preserves the Peircean operations on atoms in the sense of the Atomic Isomorphism Theorem.

Fix three arbitrary atoms in $\mathfrak{A}$ and the corresponding images, under $\vartheta$, of these three atoms in $\mathfrak{C}[\mathcal{F}]$, say

$$
a_{x y, \alpha}, \quad a_{w z, \beta}, \quad a_{u v, \gamma} \quad \text { and } \quad R_{x y, \alpha}, \quad R_{w y, \beta}, \quad R_{u v, \gamma}
$$

respectively. Treat first the case of the operation of relative multiplication. In view of (1), it is to be shown that

$$
a_{u v, \gamma} \leq a_{x y, \alpha} ; a_{w z, \beta} \quad \text { if and only if } \quad R_{u v, \gamma} \subseteq R_{x y, \alpha} \otimes R_{w z, \beta} .
$$

Lemma 6.9 and Semi-scaffold Relative Product Lemma 6.10 imply that

$$
a_{u v, \gamma} \leq a_{x y, \alpha} ; a_{w z, \beta} \quad \text { if and only if } \quad y=w, u=x, v=z,
$$

and the coset $H_{x z, \gamma}$ determined by the index $\gamma$ satisfies the inclusion

$$
H_{x z, \gamma} \subseteq \varphi_{x y}^{-1}\left[K_{x y, \alpha} ; H_{y z, \beta}\right] ; H_{x z, \zeta},
$$

where $\zeta$ is the index chosen for the triple $(x, y, z)$ so that

$$
H_{x z, \zeta} ; a_{x z} \leq a_{x y} ; a_{y z} .
$$

The definition of the operation $\otimes$ implies that

$$
R_{u v, \gamma} \subseteq R_{x y, \alpha} \otimes R_{w z, \beta} \quad \text { if and only if } \quad y=w, u=x, v=z,
$$

and the coset $H_{x z, \gamma}$ determined by the index $\gamma$ satisfies the inclusion

$$
H_{x z, \gamma} \subseteq \varphi_{x y}^{-1}\left[K_{x y, \alpha} ; H_{y z, \beta}\right] ; C_{x y z},
$$

where $C_{x y z}$ is the coset of the product group $H_{x y} ; H_{x z}$ that is defined by

$$
C_{x y z}=H_{x y} ; H_{x z, \zeta} \text {. }
$$


Observe that the inclusion in (6) is equivalent to the one in (4), because the right sides of these two inclusions are equal. In more detail,

$$
\begin{aligned}
& \varphi_{x y}^{-1}\left[K_{x y, \alpha} ; H_{y z, \beta}\right] ; C_{x y z}=\varphi_{x y}^{-1}\left[K_{x y, \alpha} ; H_{y z, \beta}\right] ; H_{x y} ; H_{x z, \zeta} \\
& \quad=\varphi_{x y}^{-1}\left[K_{x y, \alpha} ; H_{y z, \beta}\right] ; H_{x y} ; H_{x z} ; H_{x z, \zeta}=\varphi_{x y}^{-1}\left[K_{x y, \alpha} ; H_{y z, \beta}\right] ; H_{x z, \zeta},
\end{aligned}
$$

by (7), the identity property of the coset $H_{x z}$ in the quotient group $G_{x} / H_{x z}$, and the facts that $\varphi_{x y}^{-1}\left[K_{x y, \alpha} ; H_{y z, \beta}\right]$ is a coset of the product group $H_{x y} ; H_{x z}$, and this product group is the identity element in the quotient group $G_{x} /\left(H_{x y} ; H_{x z}\right)$. It follows that the condition in (4) may be replaced by the one in (6), so that the inequality on the left side of (3) and the inclusion on the left side of (5) are both equivalent to the same condition, and therefore they are equivalent to each other. This establishes (2).

Turn next to the operation of converse, with the goal of showing that

$$
a_{u v, \gamma} \leq a_{x y, \alpha}^{\smile} \quad \text { if and only if } \quad R_{u v, \gamma} \subseteq R_{x y, \alpha}^{-1} .
$$

Semi-scaffold Converse Lemma 6.8 implies that

$$
a_{u v, \gamma} \leq a_{x y, \alpha}^{\smile} \quad \text { if and only if } \quad u=y, v=x \text {, and } H_{x y, \alpha}=H_{x y, \gamma},
$$

and if the conditions on the right side of this equivalence are satisfied, then equality actually holds on the left side. Semi-frame condition (ii) implies that

$$
R_{u v, \gamma} \subseteq R_{x y, \alpha}^{-1} \quad \text { if and only if } \quad u=y, v=x \text {, and } H_{x y, \alpha}^{\smile}=H_{x y, \gamma},
$$

and if the conditions on the right side of this equivalence are satisfied, then equality actually holds. The conditions on the right sides of (10) and (11) are the same, so the inequalities on the left sides must be equivalent. This establishes (9).

Turn finally to the identity element. It is to be shown that that

$$
a_{u v, \gamma} \leq 1 \text { ' if and only if } \quad R_{u v, \gamma} \subseteq i d_{U} .
$$

Semi-scaffold Identity Lemma 6.7 implies that

$$
a_{u v, \gamma} \leq 1, \quad \text { if and only if } \quad u=v \text { and } \gamma=0 .
$$

The definition of the relation $R_{u v, \gamma}$ and semi-frame condition (i) imply that

$$
R_{u v, \gamma} \subseteq i d_{U} \quad \text { if and only if } \quad u=v \text { and } \gamma=0 .
$$

As before, the conditions on the right sides of (13) and (14) are the same, so the inequalities on the left sides must be equivalent. This proves (12).

It has been shown that the bijection $\vartheta$ satisfies the conditions of the Atomic Isomorphism Theorem. Apply that theorem to conclude that $\vartheta$ can be extended to an isomorphism from $\mathfrak{A}$ to $\mathfrak{C}[\mathcal{F}]$.

As was mentioned after Definition 5.3, not every atomic, measurable relation algebra has a scaffold, but if there is a scaffold, then a stronger result than Representation Theorem 7.2 is true. The existence of a scaffold means that for every triple $(x, y, z)$ in $\mathcal{E}_{3}$ with $x<y<z$, it is always possible to choose the atoms $a_{x y}$, $a_{y z}$ and $a_{x z}$ so that the inequality

$$
a_{x z} \leq a_{x y} ; a_{y z}
$$


holds. Consequently, the coset $H_{x y, \zeta}$ of $H_{x z}$ that is chosen to tanslate $a_{x z}$ to a position below $a_{x y} ; a_{x z}$ in the sense that

$$
H_{x z, \zeta} ; a_{x z} \leq a_{x y} ; a_{y z}
$$

may always be taken to be the identity coset $H_{x z}$, or put another way, one may always choose $\zeta=0$. The inner automorphism $\tau$ of the the quotient group $G_{x} / H_{x z}$ that is determined by this coset is then the identity automorphism of this group, and therefore the inner automorphism $\hat{\tau}$ of the quotient group $G_{x} /\left(H_{x z} ; H_{x z}\right)$ that is induced by $\tau$ is identity automorphism of its group. As a result, semi-frame condition (iv) assumes the form

$$
\hat{\varphi}_{x y} \mid \hat{\varphi}_{y z}=\hat{\varphi}_{x z} .
$$

A semi-frame satisfying this condition instead of semi-frame condition (iv) is called a frame.

Under these conditions, the shifting coset $C_{x y z}$ that is defined in terms of the coset $H_{x z, \zeta}$ becomes the identity coset

$$
C_{x y z}=H_{x y} ; H_{x z}
$$

of the quotient group $G_{x} /\left(H_{x y} ; H_{x z}\right)$, and the definition of the operator $\otimes$ between the atomic relations $R_{x y, \alpha}$ and $R_{y z, \beta}$ assumes the form

$$
R_{x y, \alpha} \otimes R_{y z, \beta}=\bigcup\left\{R_{x z, \gamma}: H_{x z, \gamma} \subseteq \varphi_{x y}^{-1}\left[K_{x y, \alpha} ; H_{y z, \beta}\right]\right\} .
$$

It was shown in Composition Theorem 3.7 of [2] that under the hypothesis of (1) and semi-frame condition (iii), the operation of relational composition between the atomic relations $R_{x y, \alpha}$ and $R_{y z, \beta}$ satisfies the equation

$$
R_{x y, \alpha} \mid R_{y z, \beta}=\bigcup\left\{R_{x z, \gamma}: H_{x z, \gamma} \subseteq \varphi_{x y}^{-1}\left[K_{x y, \alpha} ; H_{y z, \beta}\right]\right\} .
$$

The right sides of (2) and (3) are the same, so the left sides must be equal. Conclusion: when an atomic, measurable relation algebra has a scaffold, the equation

$$
R_{x y, \alpha} \otimes R_{y z, \beta}=R_{x y, \alpha} \mid R_{y z, \beta}
$$

holds, so that the defined operation $\otimes$ coincides with the set-theoretic operation of relational composition.

In this case, the coset relation algebra $\mathfrak{C}[\mathcal{F}]$ is a set relation algebra, and actually a subalgebra of the full set relation algebra with base set and unit

$$
U=\bigcup\left\{G_{x}: x \in I\right\} \quad \text { and } \quad E=\bigcup\left\{G_{x} \times G_{y}:(x, y) \in \mathcal{E}\right\}
$$

respectively. This algebra is called the group relation algebra on the frame $\mathcal{F}$ in [2], and is denoted by $\mathfrak{G}[\mathcal{F}]$.

The system of cosets

$$
C=\left\langle C_{x y z}:(x, y, z) \in \mathcal{E}_{3}\right\rangle
$$

is entirely unnecessary in this case, as are the inner automorphisms $\tau$ determined by these cosets, so instead of considering group triples $\mathcal{F}=(G, \varphi, C)$ satisfying semi-frame conditions (i)-(iv) in Definition 5.3, it suffices to consider group pairs $\mathcal{F}=(G, \varphi)$ satisfying the four frame conditions, namely conditions (i)-(iii) from Definition 5.3 and condition (1) above. This is the approach that is taken in [2].

The group pair constructed from a scaffold $a=\left\langle a_{x y}:(x, y) \in \mathcal{E}\right\rangle$ in a measurable relation algebra is called the group pair associated with a.

Theorem 7.3. (Frame Theorem) The group pair associated with a scaffold in a measurable relation algebra is always a frame. 
Notice that the hypothesis on the measurable relation algebra of being atomic is unnecessary when there is a scaffold (or even a semi-scaffold). The existence of a scaffold always implies that the measurable relation algebra under consideration is atomic, by the argument of Semi-scaffold Partition Lemma 6.6.

The preceding observations and remarks, including Frame Theorem 7.3, show that the existence of a scaffold in a measurable relation algebra implies that the semi-frame $\mathcal{F}$ associated with the given scaffold is actually a frame, and consequently the coset relation algebra $\mathfrak{C}[\mathcal{F}]$ constructed from $\mathcal{F}$ is actually the group relation algebra $\mathfrak{G}[\mathcal{F}]$, which is of course a set relation algebra. Representation Theorem 7.2 therefore assumes the the following stronger form.

Theorem 7.4 (Scaffold Representation Theorem). Every measurable relation algebra with a scaffold is essentially isomorphic to a group relation algebra.

The preceding theorem implies that a measurable relation algebra with a scaffold is representable as a set relation algebra in a stronger sense than is usually intended. To explain this stronger sense, consider an arbitrary relation algebra $\mathfrak{B}$ and its completion $\mathfrak{A}$. An isomorphism $\vartheta$ from $\mathfrak{A}$ to a complete set relation algebra $\mathfrak{C}-$ that is to say, to a set relation algebra $\mathfrak{C}$ in which the union of every set of relations in the algebra is again a relation in the algebra - must preserve all existing suprema as unions in the sense that, for every subset $X$ of $\mathfrak{A}$ with $a=\sum X$, we have

$$
\vartheta(a)=\vartheta\left(\sum X\right)=\bigcup\{\vartheta(b): b \in X\} .
$$

The reason is that isomorphisms preserve suprema, and the supremum of each subset $Y$ of $\mathfrak{C}$ is, by assumption, the union of the relations in $Y$. The restriction of $\vartheta$ to $\mathfrak{B}$ is an embedding of $\mathfrak{B}$ into $\mathfrak{C}$ and therefore a representation of $\mathfrak{B}$ as a set relation algebra, but it also inherits from $\vartheta$ the stronger property of preserving all existing suprema as unions. Indeed, if $X$ is any subset of $\mathfrak{B}$ such that the supremum $a=\sum X$ exists in $\mathfrak{B}$, then $a$ remains the supremum of $X$ in $\mathfrak{A}$, because $\mathfrak{A}$ is the completion of $\mathfrak{B}$, and therefore $\vartheta$ preserves this supremum as a union. Representations that preserve all existing suprema as unions are called complete representations, and a relation algebra with a complete representation is said to be completely representable.

Corollary 7.5. Every measurable relation algebra with a scaffold is completely representable.

It turns out that a kind of converse to Corollary 7.5 is true.

Theorem 7.6. Every measurable relation algebra that is completely representable has a scaffold.

Proof. Let $\mathfrak{A}$ be a measurable relation algebra, and assume that $\vartheta$ is a complete representation of $\mathfrak{A}$. Thus, $\vartheta$ is an embedding of $\mathfrak{A}$ into the set relation algebra of all subrelations of some equivalence relation $E$ on a base set $U$, so that

$$
\vartheta(1)=E,
$$

and $\vartheta$ preserves all existing suprema in $\mathfrak{A}$ as unions A completely representable relation algebra is always atomic (see [5]), so $\mathfrak{A}$ must be atomic. Take $X$ to be the set of atoms in $\mathfrak{A}$. Each element $d$ in $\mathfrak{A}$ is the sum of the atoms below it,

$$
d=\sum\{a \in X: a \leq d\}
$$


and therefore

$$
\vartheta(d)=\bigcup\{\vartheta(a): a \in X \text { and } a \leq d\},
$$

by the assumption that the representation $\vartheta$ is complete.

The set $\mathcal{E}$ of pairs $(x, y)$ of measurable atoms such that $x ; 1 ; y \neq 0$, or equivalently, such that

$$
1 ; x ; 1=1 ; y ; 1,
$$

is an equivalence relation on the set of measurable atoms. Let $\left\langle w_{\xi}: \xi \leq \kappa\right\rangle$ be a system of representatives for the equivalence classes, so that each equivalence class of $\mathcal{E}$ contains exactly one $w_{\xi}$.

The relations $\vartheta\left(w_{\xi}\right)$ are non-empty, because the elements $w_{\xi}$ are atoms, and they are included in the equivalence relation $E$, which is the unit of the representing algebra. It follows that each relation of the form

$$
E\left|\vartheta\left(w_{\xi}\right)\right| E
$$

is a non-empty union of components of $E$, that is to say, it is a non-empty union of relations of the form $V \times V$, where each $V$ is an equivalence class of $E$. In more detail, if $(p, q)$ is a pair in $\vartheta\left(w_{\xi}\right)$, then $p$ and $q$ are in the same equivalence class of $E$, call it $V$. If $r$ and $s$ are any other elements in $V$, then the pairs $(r, p)$ and $(q, s)$ are both in $E$, and therefore the pair $(r, s)$ must be in (4), by the definition of relational composition. Thus, every pair in $V \times V$ belongs to (4). For each representative $w_{\xi}$, choose an equivalence class $V_{\xi}$ of $E$ such that

$$
V_{\xi} \times V_{\xi} \subseteq E\left|\vartheta\left(w_{\xi}\right)\right| E .
$$

Consider now an arbitrary measurable atom $x$. Let $w_{\xi}$ be the representative of $x$, so that

$$
1 ; x ; 1=1 ; w_{\xi} ; 1,
$$

by the definition of $\mathcal{E}$. Observe that

$$
\begin{aligned}
E|\vartheta(x)| E=\vartheta(1)|\vartheta(x)| \vartheta(1)=\vartheta(1 ; x ; 1) & =\vartheta\left(1 ; w_{\xi} ; 1\right) \\
& =\vartheta(1)\left|\vartheta\left(w_{\xi}\right)\right| \vartheta(1)=E\left|\vartheta\left(w_{\xi}\right)\right| E,
\end{aligned}
$$

by (1), the representation properties of $\vartheta$, and (6). It follows from this computation, the choice of $w_{\xi}$, and (5) that

$$
V_{\xi} \times V_{\xi} \subseteq E|\vartheta(x)| E .
$$

Since $x$ is assumed to be a measurable atom in $\mathfrak{A}$, we have $0 \neq x \leq 1$, and therefore

$$
\varnothing \neq \vartheta(x) \subseteq \vartheta\left(1^{\prime}\right)=i d_{U},
$$

by the representation properties of $\vartheta$. Use this observation and (7) to choose an element $p_{x}$ in $V_{\xi}$ wih the property that the pair $\left(p_{x}, p_{x}\right)$ belongs to the relation $\vartheta(x)$.

Consider next an arbitrary pair $(x, y)$ in $\mathcal{E}$. The elements $x$ and $y$ are, by definition, in the same equivalence class of $\mathcal{E}$, and therefore they have the same representative, say $w_{\xi}$. The elements $p_{x}$ and $p_{y}$ are both chosen to be in $V_{\xi}$, so the pair $\left(p_{x}, p_{y}\right)$ belongs to the component $V_{\xi} \times V_{\xi}$, and therefore also to the unit relation $E$. The pairs $\left(p_{x}, p_{x}\right)$ and $\left(p_{y}, p_{y}\right)$ are chosen to be in $\vartheta(x)$ and $\vartheta(y)$ respectively, so the pair $\left(p_{x}, p_{y}\right)$ belongs to the relation

$$
\vartheta(x)|E| \vartheta(y)
$$


by the definition of relational composition. Since

$$
\vartheta(x ; 1 ; y)=\vartheta(x)|\vartheta(1)| \vartheta(y)=\vartheta(x)|E| \vartheta(y),
$$

by the representation properties of $\vartheta$ and(1), it follows that

$$
\left(p_{x}, p_{y}\right) \in \vartheta(x ; 1 ; y) \text {. }
$$

We now come to the heart of the argument. The element $x ; 1 ; y$ is non-zero, by the definition of $\mathcal{E}$, so it must be the sum of a non-empty set of atoms. In view of (8) and (2) (with $x ; 1 ; y$ in place of $d$ ), there must be an atom below $x ; 1 ; y$ whose image under $\vartheta$ contains the pair $\left(p_{x}, p_{y}\right)$. Moreover, this atom is unique, because (2) is a disjoint union of images of mutually distinct atoms. Call the atom $a_{x y}$. In other words, $a_{x y}$ is the unique atom in $\mathfrak{A}$ with the property that

$$
\left(p_{x}, p_{y}\right) \in \vartheta\left(a_{x y}\right) .
$$

We shall show that the system

$$
\left\langle a_{x y}:(x, y) \in \mathcal{E}\right\rangle
$$

is a scaffold.

First of all, $x$ is an atom below $x ; 1 ; x$, and the pair $\left(p_{x}, p_{x}\right)$ belongs to $\vartheta(x)$, by the choice of the element $p_{x}$. On the hand, $a_{x x}$ is defined to be the unique atom below $x ; 1 ; x$ with the property that its image under $\vartheta$ contains the pair $\left(p_{x}, p_{x}\right)$. Consequently,

$$
a_{x x}=x .
$$

Second, $a_{x y}$ is an atom below $x ; 1 ; y$, so its converse $a_{x y}$ is an atom below $y ; 1 ; x$, by Lemmas 2.1(vi) and 2.5(iii), and monotony. Moreover, the pair $\left(p_{y}, p_{x}\right)$ belongs to the image $\vartheta\left(a_{x y}\right)$, because the pair $\left(p_{x}, p_{y}\right)$ belongs to $\vartheta\left(a_{x y}\right)$, by definition, and

$$
\vartheta\left(a_{x y}^{-}\right)=\vartheta\left(a_{x y}\right)^{-1}
$$

by the representation properties of $\vartheta$. On the other hand, the element $a_{y x}$ is defined to be the unique atom below $y ; 1 ; x$ whose image under $\vartheta$ contains the pair $\left(p_{y}, p_{x}\right)$, so it follows that

$$
a_{y x}=a_{x y}^{\smile} .
$$

Finally, the pairs $\left(p_{x}, p_{y}\right)$ and $\left(p_{y}, p_{z}\right)$ are in $\vartheta\left(a_{x y}\right)$ and $\vartheta\left(a_{y z}\right)$ respectively, by (9), so the pair $\left(p_{x}, p_{z}\right)$ is in the relational composition $\vartheta\left(a_{x y}\right) \mid \vartheta\left(a_{x y}\right)$, by the definition of relational composition. Since

$$
\vartheta\left(a_{x y}\right) \mid \vartheta\left(a_{y z}\right)=\vartheta\left(a_{x y} ; a_{y z}\right),
$$

it follows that the pair $\left(p_{x}, p_{z}\right)$ belongs to the relation $\vartheta\left(a_{x y} ; a_{y z}\right)$. The same pair also belongs to the relation $\vartheta\left(a_{x z}\right)$, by (9) so the two relations have a non-empty intersection. Use the representation properties of $\vartheta$ to see that the elements $a_{x y} ; a_{y z}$ and $a_{x z}$ cannot be disjoint. Since $a_{x z}$ is an atom, it follows that

$$
a_{x z} \leq a_{x y} ; a_{y z} .
$$

Equations (11)-(13) are just the conditions required for (10) to be a scaffold.

When the unit element $E$ of the representation coincides with the universal relation $U \times U$, the steps leading up to (8) in the preceding proof are unnecessary. In this case, the equivalence relation $\mathcal{E}$ consists of all pairs of measurable atoms. For each measurable atom $x$, choose an element $p_{x}$ in $U$ such that the pair $\left(p_{x}, p_{x}\right)$ 
is in $\vartheta(x)$. Certainly, all of the pairs $\left(p_{x}, p_{y}\right)$ are in $U \times U$, that is, in $\vartheta(1)$. Because the pairs $\left(p_{x}, p_{x}\right)$ and $\left(p_{y}, p_{y}\right)$ are in $\vartheta(x)$ and $\vartheta(y)$ respectively, the pair $\left(p_{x}, p_{y}\right)$ must be in

$$
\vartheta(x)|\vartheta(1)| \vartheta(y) .
$$

Thus, we obtain (8) directly. The remainder of the proof is just as before.

The next corollary is a direct consequence of the preceding theorem and Scaffold Isomorphism Theorem 7.4.

Corollary 7.7. Every measurable relation algebra that is completely representable is essentially isomorphic to a group relation algebra.

A finite relation algebra is necessarily complete, and any representation of it is necessarily a complete representation. Thus, the preceding corollary can be given a strong formulation when the algebra in question is finite.

Corollary 7.8. Every measurable relation algebra that is finite and representable is isomorphic to a group relation algebra.

\section{Finitely measurable Relation algebras}

Atomic Partition Lemma 4.11 raises several question. For example, under what conditions will there be at least one atom below a given rectangle $x ; 1 ; y$ when the sides $x$ and $y$ are measurable atoms? Speaking more broadly, under what conditions will a measurable relation algebra automatically be atomic? It turns out that finite measurability implies atomicity. In other words, if the group $G_{x}$ is finite for every measurable atom $x$ in a measurable relation algebra, then the algebra is automatically atomic. Keep in mind that finitely measurable relation algebras may be infinite in size, because there may be infinitely many measurable atoms in the algebra.

The key observation is contained in the following lemma, which could have been proved immediately after Product Lemma 4.13. Recall that the set $X_{a}$ is a union of cosets of $H_{a}$. If it is the union of only finitely many such cosets, then we shall say that $H_{a}$ has finite index in $X_{a}$. This terminology parallels that of group theory, where one speaks of the index of a subgroup in a group.

Lemma 8.1. Let $x$ and $y$ be measurable atoms, and $0<a \leq x ; 1 ; y$. If $H_{a}$ has finite index in $X_{a}$, then there is left-regular element below a.

Proof. Consider the collection of subsets $Z$ of $X_{a}$ with the following properties. First, $x$ is in $Z$. Second,

$$
\prod_{f \in Z} f ; a \neq 0 \text {. }
$$

Because $f ; a=g ; a$ if and only if $f$ and $g$ are in the same coset of $H_{a}$, by Corollary 3.10, it may also be assumed that $Z$ is a union of cosets of $H_{a}$.

There are only finitely many cosets of $H_{a}$ included in $X_{a}$, by assumption, and each $Z$ under consideration is assumed to be a union of some of these cosets, so there are only finitely many possible choices for $Z$. Moreover, there certainly exist sets $Z$ with the required properties. For instance, the left stabilizer $H_{a}$ is such a set. In more detail, the identity element $x$ is in $H_{a}$, because $H_{a}$ is a subgroup of $G_{x}$. Also,

$$
\prod_{f \in H_{a}} f ; a=a \neq 0,
$$


because $H_{a}$ is the left stabilizer of $a$. Finally, $H_{a}$ is the union of the singleton coset $\left\{H_{a}\right\}$.

As there are only finitely many choices for the set $Z$, it is possible to choose one of maximal cardinality. Let $Z$ be such a maximal choice, and write

$$
b=\prod_{f \in Z} f ; a,
$$

with the goal of showing that $b$ is a left-regular element below $a$. First of all, $b$ is not 0 , by the second property. Also, $x$ is in $Z$, by the first property, so $x ; a$ is one of the elements in the product (1). Lemma 2.3(iii) implies that $x ; a=a$, so $a$ must be one of the elements in in the product (1), and therefore $b$ must be below $a$, by Boolean algebra. It remains to show that $b$ is left-regular. This amounts to proving that

$$
X_{b}=H_{b},
$$

by Corollary 4.3.

The inclusion from right to left in (2) is a consequence of Lemma 4.5(ii). To establish the reverse inclusion, consider an element $g$ in $X_{b}$, with the goal of showing that $g$ is in $H_{b}$. Observe that (1) is essentially a finite product. Use (1) and the distributive law for functions to obtain

$$
g ; b=g ;\left(\prod_{f \in Z} f ; a\right)=\prod_{f \in Z} g ; f ; a .
$$

The product $(g ; b) \cdot b$ is non-zero, by Lemma 4.5 (i) (with $g$ and $b$ in place of $f$ and $a$ respectively), because $g$ is assumed to belong to $X_{b}$. Consequently,

$$
\left(\prod_{f \in Z} g ; f ; a\right) \cdot\left(\prod_{f \in Z} f ; a\right)=(g ; b) \cdot b \neq 0,
$$

by (3) and (1). Use (3), (4), and the assumed maximality of the cardinality of $Z$ to conclude that every element in the set

$$
g ; Z=\{g ; f: f \in Z\}
$$

must also belong to $Z$, that is to say,

$$
g ; Z \subseteq Z \text {. }
$$

For each coset $H_{\xi}$ of $H_{a}$ that is included in $Z$, the coset $g ; H_{\xi}$ is included in $g ; Z$, by (5), and these cosets are distinct for distinct $\xi$, by the cancellation law for groups. Consequently, the sets $g ; Z$ and $Z$ are unions of the same finite number of cosets of $H_{a}$. Use this observation and (6) to arrive at $g ; Z=Z$, from which it follows that

$$
g ; b=\prod_{f \in Z} g ; f ; a=\prod_{f \in Z} f ; a=b,
$$

by (3) and (1). This proves that $g$ is in the left stabilizer $H_{b}$, so (2) holds and therefore $b$ is left-regular.

Lemma 8.2. For any measurable atoms $x$ and $y$, and any non-zero element $a \leq$ $x ; 1 ; y$, if the set $X_{a}$ is finite, then there is an atom below $a$.

Proof. Observe first that if $b \leq a$, then $X_{b} \subseteq X_{a}$. Indeed, if $b \leq a$, then the monotony laws imply that $b^{\smile} \leq a^{\smile}$ and therefore that $b ; b^{\smile} \leq a ; a^{\smile}$. Consequently,

$$
\sum X_{b}=b ; b^{-} \leq a ; a^{\smile}=\sum X_{a},
$$

by definition of the sets $X_{a}$ and $X_{b}$. As these are sets of atoms, it follows that $X_{b}$ is included in $X_{a}$. 
Let $W$ be the set of left-regular elements below $a$. For every element $b$ in $W$,

$$
H_{b}=X_{b} \subseteq X_{a},
$$

by Corollary 4.3 and the initial observation of the proof, so the stabilizer of every element in $W$ is finite. Moreover, the set $W$ is not empty, by Lemma 8.1. It is therefore possible to choose an element $b$ in $W$ such that its left stabilizer $H_{b}$ has minimal finite cardinality among the left stabilizers of elements in $W$. Clearly,

$$
0<b \leq a
$$

by Lemma 4.4 and the definition of $W$.

The argument that $b$ must be an atom proceeds by contraposition. If $b$ is not an atom, then there is a non-zero element $c$ that is strictly below $b$, by Boolean algebra and the first inequality in (1). Obviously, $c$ is also below $a$, by the second inequality in (1), so the set $X_{c}$ is included in the set $X_{a}$, by the initial observation of the proof (with $c$ in place of $b$ ). In particular, the set $X_{c}$ must be finite, since this is true of $X_{a}$. Apply Lemma 8.1 (with $c$ in place of $a$ ) to obtain a left-regular element $d$ below $c$. It follows from the definition of the set $W$ that $d$ belongs to this set. Both $b$ and $d$ are left-regular elements, and

$$
0<d=b \cdot d \leq c<b
$$

by the choices of the elements $c$ and $d$, together with Lemma 4.4. These inequalities and Lemma 4.14 imply that the left stabilizer $H_{d}$ must be a proper subgroup of the finite left stabilizer $H_{b}$. Consequently, the cardinality of $H_{b}$ cannot be minimal among the left stabilizers of elements $b$ in $W$.

Theorem 8.3. Every finitely measurable relation algebra is atomic.

Proof. Let $a$ be an arbitrary non-zero element in the relation algebra, with the goal of showing that there is an atom below $a$. Consider the set $I$ of measurable atoms. Each element $x$ in $I$ is finitely measurable, by assumption, so the corresponding group $G_{x}$ is finite, by the definition of finite measurability. The identity element 1' is the sum of the set $I$, by the assumption of measurability, so

$$
1=1^{\prime} ; 1 ; 1^{\prime}=\left(\sum I\right) ; 1 ;\left(\sum I\right)=\sum\{x ; 1 ; y: x, y \in I\},
$$

by the complete distributivity of relative multiplication. It follows from (1) and Boolean algebra that

$$
a=a \cdot 1=\sum\{a \cdot(x ; 1 ; y): x, y \in I\}
$$

The element $a$ is assumed to be different from zero, so (2) implies that there must be measurable atoms $x$ and $y$ in $I$ such that the element

$$
d=a \cdot(x ; 1 ; y)
$$

is not 0 . The set $X_{d}$ is a subset of the finite group $G_{x}$ and is therefore itself a finite set. Apply Lemma 8.2 (with $d$ in place of $a$ ) to obtain an atom $b$ below $d$. Clearly, $b$ is also an atom below $a$. 


\section{A CHARACTERIZATION OF REGULAR ELEMENTS}

The next theorem and its corollary characterize regular elements in several illuminating ways. One consequence of the corollary is that, in the presence of atoms, left-regularity implies right-regularity and conversely. In other words, left-regular (or right-regular) elements are regular. Moreover, regular elements are precisely the elements that can be written in the form $\sum M$; $a$ for some atom $a$ below $x ; 1 ; y$ and some subgroup $M$ of $G_{x}$ that includes $H_{a}$. Equivalently, regular elements are precisely the elements that can be written in the form $\sum M_{\xi} ; a$ for some atom $a$ and some coset $M_{\xi}$ of a subgroup $M$ of $G_{x}$ that includes $H_{a}$.

Theorem 9.1 (Regular Characterization Theorem). Let $x$ and $y$ be measurable atoms, and $a \leq x ; 1 ; y$ a regular element with normal stabilizers. For each element $b \leq x ; 1 ; y$ with $H_{a} \subseteq H_{b}$, the following conditions are equivalent.

(i) $b$ is left-regular.

(ii) $b$ is regular.

(iii) $b=\sum M_{\zeta}$; a for some coset $M_{\zeta}$ of a subgroup $M$ of $G_{x}$ such that $H_{a} \subseteq M$.

(iv) $b=\sum M$; c for some subgroup $M$ of $G_{x}$ such that $H_{a} \subseteq M$ and some (any) left translation $c$ of a that is below $b$.

(v) $b=\sum H_{b, \rho} ;$ a for some coset $H_{b, \rho}$ of $H_{b}$.

(vi) $b=\sum H_{b}$; c for some (any) left translation $c$ of a that is below $b$.

Proof. Obviously, (ii) implies (i). To establish the implication from (i) to (vi), assume that $b$ is left-regular. The assumptions that $a$ is regular with normal stabilizers, and that $H_{a} \subseteq H_{b}$, mean that the implication from (i) to (iii) in Translation Lemma 4.17 may be applied to obtain a left translation $c$ of $a$ that is below $b$. Any such left translation $c$ is a left-regular element with the same normal stabilizer $H_{a}$ as $a$, by Translation Lemmas 4.15 and 4.17, and the assumptions on $a$. Apply Partition Lemma 4.12 (with $c$ and $H_{b}$ in place of $a$ and $H_{b, \eta}$ respectively), and use the definition of $H_{b}$ as the stabilizer of $b$, to conclude that

$$
\sum H_{b} ; c=H_{b} ; b=b,
$$

as desired.

To prove that (vi) implies (v), assume that

$$
c=H_{a, \rho} ; a \quad \text { and } \quad b=\sum H_{b} ; c
$$

for some coset $H_{a, \rho}$ of $H_{a}$. The set defined by

$$
H_{b, \rho}=H_{b} ; H_{a, \rho}
$$

is a coset of $H_{b}$, because the assumption $H_{a} \subseteq H_{b}$ implies that every element in $H_{a, \rho}$ gives rise to the same coset of $H_{b}$. Use (1) and (2) to arrive at

$$
b=\sum H_{b} ; c=\sum H_{b} ; H_{a, \rho} ; a=\sum H_{b, \rho} ; a .
$$

This proves $(\mathrm{v})$.

To see that (v) implies (iii), take

$$
M=H_{b} \quad \text { and } \quad M_{\zeta}=H_{b, \rho},
$$

and use the assumption that $H_{a} \subseteq H_{b}$.

To establish the implications from (iii) to (iv) and from (iv) to (ii), it is helpful to introduce some notation. Let $M$ be a subgroup of $G_{x}$ that includes $H_{a}$. Fix left 
coset systems

$$
\left\langle H_{\xi}: \xi<\kappa\right\rangle \quad \text { and } \quad\left\langle M_{\eta}: \eta<\lambda\right\rangle
$$

for $H_{a}$ and $M$ respectively in $G_{x}$. The assumption $H_{a} \subseteq M$ implies the existence of a partition $\left\langle\Gamma_{\eta}: \eta<\lambda\right\rangle$ of the index set $\kappa$ such that

$$
M_{\eta}=\bigcup_{\xi \in \Gamma_{\eta}} H_{\xi}
$$

for each $\eta<\lambda$.

We now take up the implication from (iii) to (iv). If (iii) holds for the subgroup $M$, then

$$
b=\sum M_{\zeta} ; a=\sum\left(\bigcup_{\xi \in \Gamma_{\zeta}} H_{\xi}\right) ; a=\sum_{\xi \in \Gamma_{\zeta}} H_{\xi} ; a
$$

by (3) (with $\zeta$ in place of $\eta$ ) and complete distributivity. The equality of the first and last terms in (4), and Partition Lemma 4.9, together imply that the elements $H_{\xi} ; a$ with $\xi$ in $\Gamma_{\zeta}$ are precisely the left translations of $a$ that are below $b$. Take $c$ to be any one of these translations, say $c=H_{\xi} ; a$. Certainly $c$ is below $b$, by (4) and the definition of $c$. The assumption in (3) (with $\zeta$ in place of $\eta$ ) implies that every element in $H_{\xi}$ gives rise to the same coset of $M$ as every element in $M_{\zeta}$, and of course that coset is $M_{\zeta}$. Consequently,

$$
M ; H_{\xi}=M ; M_{\zeta}=M_{\zeta} .
$$

Use (4) and (5) to conclude that

$$
b=\sum M_{\zeta} ; a=\sum M ; H_{\xi} ; a=\sum M ; c,
$$

as desired.

Turn, finally, to the implication from (iv) to (ii). The element $a$ is assumed to be regular with normal stabilizers, and $c$ is assumed to be a left translation of $a$, so $c$ must be a regular element with the same normal stabilizers as $a$, by Translation Lemma 4.15(i) and its right-regular version. In particular,

$$
H_{c}=H_{a} \text {. }
$$

Isomorphism Theorem 5.4 guarantees the existence of an isomorphism $\varphi_{c}$ from $G_{x} / H_{c}$ to $G_{y} / K_{c}$ with the property that, writing $K_{\xi}=\varphi_{c}\left(H_{\xi}\right)$, we have

$$
H_{\xi} ; c=c ; K_{\xi},
$$

for each $\xi<\kappa$. Take $\eta=0$ in (3), and use the convention $M_{0}=M$, to obtain

$$
M=\bigcup_{\xi \in \Gamma_{0}} H_{\xi} .
$$

Put

$$
N=\bigcup_{\xi \in \Gamma_{0}} K_{\xi}
$$

The subgroup $M$ of $G_{x}$ is assumed in (iv) to include the subgroup $H_{a}$, so it also includes $H_{c}$, by (6). Use group theory, the definition of a quotient set and (8) to see that the quotient

$$
M / H_{c}=\left\{f / H_{c}: f \in M\right\}=\left\{H_{\xi}: \xi \in \Gamma_{0}\right\}
$$

is a subgroup of $G_{x} / H_{c}$. The image of this subgroup under the quotient isomorphism $\varphi_{c}$ is the set

$$
\begin{aligned}
\varphi_{c}\left(M / H_{c}\right)=\left\{\varphi_{c}\left(H_{\xi}\right): \xi \in \Gamma_{0}\right\}=\left\{K_{\xi}: \xi \in \Gamma_{0}\right\} & \\
& =\left\{g / K_{c}: g \in N\right\}=N / K_{c}
\end{aligned}
$$


by the definition of an image set, (10), the definition of $\varphi_{c},(9)$, and the definition of the set $N / K_{c}$. Isomorphisms preserve the property of being a subgroup, so the equality of the first and last terms in (11) implies that $N / K_{c}$ is a subgroup of $G_{y} / K_{c}$. It follows from group theory that $N$ must be a subgroup of $G_{y}$ that includes $K_{c}$.

Use (8), complete distributivity, (7), complete distributivity, and (9) to obtain

$$
\begin{aligned}
\sum M ; c=\sum\left(\bigcup_{\xi \in \Gamma_{0}} H_{\xi}\right) ; c & =\sum_{\xi \in \Gamma_{0}} H_{\xi} ; c \\
& =\sum_{\xi \in \Gamma_{0}} c ; K_{\xi}=\sum c ;\left(\bigcup_{\xi \in \Gamma_{0}} K_{\xi}\right)=\sum c ; N .
\end{aligned}
$$

Use the assumption in (iv), the second involution law, complete distributivity, the associative law, the regularity of $c$, and the assumption that $M$ is a subgroup of $G_{x}$ (and hence closed under formation of converses and relative products), and $H_{c} \subseteq M$ to get

$$
\begin{aligned}
& b ; b^{\smile}=\left(\sum M ; c\right) ;\left(\sum M ; c\right)^{-}=\left(\sum M ; c\right) ;\left(\sum c^{\smile} ; M^{-}\right) \\
& =\sum M ; c ; c^{\smile} ; M^{\smile}=\sum M ; H_{c} ; M^{\smile}=\sum M .
\end{aligned}
$$

A similar computation using (iv), the second involution law, complete distributivity, the associative law, the group properties of $M,(12)$, the regularity of $c$, and the fact proved above that $N$ is a subgroup of $G_{y}$ that includes $K_{c}$, yields

$$
\begin{aligned}
& b^{\smile} ; b=\left(\sum M ; c\right)^{\smile} ;\left(\sum M ; c\right)=\left(\sum c^{\smile} ; M^{\smile}\right) ;\left(\sum M ; c\right) \\
& =\sum c^{\smile} ; M^{\smile} ; M ; c=\sum c^{\smile} ; M ; c \\
& \quad=\sum c^{-} ; c ; N=\sum K_{c} ; N=\sum N .
\end{aligned}
$$

In view of (13), (14), and the definition of regularity, the proof that $b$ is regular will be complete once it is shown that

$$
M=H_{b} \quad \text { and } \quad N=K_{b} .
$$

If $f$ is in $M$, then $f ; M=M$, since $M$ is a group, and therefore

$$
f ; b=f ;\left(\sum M ; c\right)=\sum f ; M ; c=\sum M ; c=b,
$$

by (iv) and complete distributivity. Thus, every element in $M$ belongs to the left stabilizer of $b$, so $M \subseteq H_{b}$. To establish the reverse inclusion, observe that

$$
\sum H_{b} \leq \sum X_{b}=b ; b^{\smile}=\sum M,
$$

by Lemma 4.5(ii) and monotony, the definition of the set $X_{b}$, and (13). The sets being summed on the left and on the right are sets of atoms, so the inequality in (16) implies the inclusion $H_{b} \subseteq M$.

The proof of the second equation in (15) is entirely analogous, but uses (14) instead of (13).

Corollary 9.2. Let $x$ and $y$ be measurable atoms. If there is an atom below $x ; 1 ; y$, then for each element $b \leq x ; 1 ; y$ the following conditions are equivalent.

(i) $b$ is left-regular.

(ii) $b$ is regular.

(iii) $b=\sum M_{\zeta} ;$ for some atom $a \leq x ; 1 ; y$ and some left coset $M_{\zeta}$ of a subgroup $M$ of $G_{x}$ such that $H_{a} \subseteq M$.

(iv) $b=\sum M ; a$ for some atom $a \leq x ; 1 ; y$ and some subgroup $M$ of $G_{x}$ such that $H_{a} \subseteq M$. 
(v) $b=\sum H_{b, \rho} ;$ a for some atom $a \leq x ; 1 ; y$ and some coset $H_{b, \rho}$ of $H_{b}$.

(vi) $b=\sum H_{b} ; a$ for some atom $a \leq x ; 1 ; y$.

Proof. It may be assumed that $b$ is non-zero, since this is implied by each of the conditions (i)-(vi). The hypothesis that there is an atom below the rectangle $x ; 1 ; y$ implies that this rectangle is a sum of atoms, by Atomic Partition Lemma 4.11. In particular, there must be an atom $a$ below $b$, since

$$
0<b \leq x ; 1 ; y \text {. }
$$

Of course, $H_{a} \subseteq H_{b}$, by Lemma 4.8. Atoms are regular elements with normal stabilizers, by Corollaries 4.7 and 4.16, so the hypotheses of Theorem 9.1 are satisfied. The equivalence of statements (i)-(vi) in that theorem, and the fact that any translation of an atom is itself an atom, by Lemma 4.11, immediately yield the corollary.

Corollary 9.3. Let $x$ and $y$ be measurable atoms, and $a \leq x ; 1 ; y$ a regular element with normal stabilizers. If $b=\sum M_{\zeta}$; a for some coset $M_{\zeta}$ of a subgroup $M$ of $G_{x}$ such that $H_{a} \subseteq M$, then $M$ is the left stabilizer of $b$.

Proof. If $b=\sum M_{\zeta} ; a$, then for some (any) translation $c$ of $a$ that is below $b$, we have

$$
b=\sum M ; c \quad \text { and } \quad b=\sum H_{b} ; c,
$$

by the implications in Theorem 9.1 from (iii) to (iv) and from (iii) to (vi) respectively. Therefore, $M=H_{b}$, by Corollary 4.10 (with $M, H_{b}$, and $c$ in place of $X, Y$, and $a$ respectively).

The two previous corollaries show that, in general, one cannot expect the stabilizers of regular elements to be normal subgroups. To see this, suppose that $a$ is an atom below $x ; 1 ; y$. Take $M$ to be any subgroup of $G_{x}$ that includes $H_{a}$ but is not normal. The element $b=\sum M ; a$ is regular, by Corollary 9.2 , and its left stabilizer is the non-normal subgroup $M$, by Corollary 9.3.

\section{REFERENCES}

[1] Andréka, H. and Givant, S., Coset relation algebras, Algebra Universalis 79,2 (2018), paper 28, 53pp. Sharit link: https://rdcu.be/L94h, arXiv:1804.00279 [math.LO]

[2] Givant, S., Relation algebras and groups, Algebra Universalis 79,2 (2018), paper 16, 38pp. Sharit link: https://rdcu.be/LJ2Y, arXiv:1804.00076 [math.LO]

[3] Givant, S., Introduction to relation algebras, Springer International Publishing AG, Cham, 2017.

[4] Givant, S. and Andréka, H., Groups and algebras of relations, The Bulletin of Symbolic Logic, 8 (2002), pp. 38-64.

[5] Hirsch, R. and Hodkinson, I., Complete representations in algebraic logic, Journal of Symbolic Logic 62 (1997), pp. 816-847.

[6] Hirsch, R. and Hodkinson, I., Relation algebras by games, Studies in Logic and the Foundations of Mathematics, vol. 147, Elsevier Science, North-Holland Publishing Company, Amsterdam, 2002, $712 \mathrm{pp}$.

[7] Jónsson, B. and Tarski, A., Boolean algebras with operators. Part I, American Journal of Mathematics 73 (1951), pp. 891-939.

[8] Jónsson, B. and Tarski, A., Boolean algebras with operators. Part II, American Journal of Mathematics 74 (1952), pp. 127-162.

[9] Maddux, R. D., Pair-dense relation algebras, Transactions of the American Mathematical Society 328 (1991), pp. 83-131. 
[10] Maddux, R. D., Relation algebras, Studies in Logic and the Foundations of Mathematics, vol. 150, Elsevier Science, North-Holland Publishing Company, Amsterdam, 2006, xxvi + $731 \mathrm{pp}$.

[11] Monk, J. D., Completions of Boolean algebras with operators, Mathematische Nachrichten 46 (1970), pp. 47-55.

Steven Givant, Mills College, 5000 MacArthur Boulevard, Oakland, CA 94613

E-mail address: givant@mills.edu

Hajnal Andréka, Alfréd Rényi Institute of Mathematics, Hungarian Academy of Sciences, ReÁltanoda utca 13-15, Budapest, 1053 Hungary

E-mail address: andreka.hajnal@renyi.mta.hu 